\title{
MIPAS: an instrument for atmospheric and climate research
}

\author{
H. Fischer ${ }^{1}$, M. Birk ${ }^{2}$, C. Blom ${ }^{1}$, B. Carli ${ }^{3}$, M. Carlotti ${ }^{4}$, T. von Clarmann ${ }^{1}$, L. Delbouille ${ }^{5}$, A. Dudhia ${ }^{6}$, D. Ehhalt ${ }^{7}$, \\ M. Endemann ${ }^{8}$, J. M. Flaud ${ }^{9}$, R. Gessner ${ }^{10}$, A. Kleinert ${ }^{1}$, R. Koopman ${ }^{8}$, J. Langen ${ }^{8}$, M. López-Puertas ${ }^{11}$, P. Mosner ${ }^{10}$,

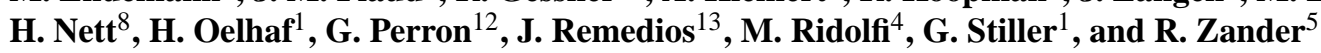 \\ ${ }^{1}$ Forschungszentrum Karlsruhe und Univ. Karlsruhe, Institute for Meteorology and Climate Research, Karlsruhe, Germany \\ ${ }^{2}$ Deutsches Zentrum für Luft- und Raumfahrt, Germany \\ ${ }^{3}$ Istituto di Fisica Applicata, N. Carrara (IFAC) del Consiglio Nazionale delle Ricerche (CNR), Firenze, Italy \\ ${ }^{4}$ Dipartimento di Chimica Fisica e Inorganica - University of Bologna, Bologna, Italy \\ ${ }^{5}$ Groupe Infra-Rouge de Physique Atmosphérique et Solaire (GIRPAS), Département AGO, Université de Liège, Belgium \\ ${ }^{6}$ Atmospheric, Oceanic and Planetary Physics, Clarendon Laboratory, Oxford University, UK \\ ${ }^{7}$ Forschungszentrum Jülich, Institut für Atmosphärische Chemie, (ICG3), Jülich, Germany \\ ${ }^{8}$ ESA/ESRIN, Frascati, Italy and ESA/ESTEC, Noordwijk, The Netherlands \\ ${ }^{9}$ Laboratoire Interuniversitaire des Systèmes Atmosphériques (LISA), CNRS, Université Paris VII et XII, France \\ ${ }^{10}$ EADS Astrium GmbH, Friedrichshafen und München, Germany \\ ${ }^{11}$ Instituto de Astrofísica de Andalucía (IAA), Granada, Spain \\ ${ }^{12}$ ABB Bomem Inc., Quebec, Canada \\ ${ }^{13}$ Earth Observation Science (EOS), Space Research Centre, Department of Physics and Astronomy, Univ. of Leicester, UK
}

Received: 1 June 2007 - Published in Atmos. Chem. Phys. Discuss.: 25 June 2007

Revised: 23 January 2008 - Accepted: 22 February 2008 - Published: 16 April 2008

\begin{abstract}
MIPAS, the Michelson Interferometer for Passive Atmospheric Sounding, is a mid-infrared emission spectrometer which is part of the core payload of ENVISAT. It is a limb sounder, i.e. it scans across the horizon detecting atmospheric spectral radiances which are inverted to vertical temperature, trace species and cloud distributions. These data can be used for scientific investigations in various research fields including dynamics and chemistry in the altitude region between upper troposphere and lower thermosphere.
\end{abstract}

The instrument is a well calibrated and characterized Fourier transform spectrometer which is able to detect many trace constituents simultaneously. The different concepts of retrieval methods are described including multi-target and two-dimensional retrievals. Operationally generated data sets consist of temperature, $\mathrm{H}_{2} \mathrm{O}, \mathrm{O}_{3}, \mathrm{CH}_{4}, \mathrm{~N}_{2} \mathrm{O}, \mathrm{HNO}_{3}$, and $\mathrm{NO}_{2}$ profiles. Measurement errors are investigated in detail and random and systematic errors are specified. The results are validated by independent instrumentation which has been operated at ground stations or aboard balloon gondolas and aircraft. Intercomparisons of MIPAS measurements with other satellite data have been carried out, too. As a result, it

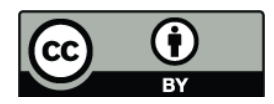

Correspondence to: H. Fischer (herbert.fischer@imk.fzk.de) has been proven that the MIPAS data are of good quality.

MIPAS can be operated in different measurement modes in order to optimize the scientific output. Due to the wealth of information in the MIPAS spectra, many scientific results have already been published. They include intercomparisons of temperature distributions with ECMWF data, the derivation of the whole $\mathrm{NO}_{\mathrm{y}}$ family, the study of atmospheric processes during the Antarctic vortex split in September 2002, the determination of properties of Polar Stratospheric Clouds, the downward transport of $\mathrm{NO}_{\mathrm{x}}$ in the middle atmosphere, the stratosphere-troposphere exchange, the influence of solar variability on the middle atmosphere, and the observation of Non-LTE effects in the mesosphere.

\section{Introduction}

MIPAS, the Michelson Interferometer for Passive Atmospheric Sounding, is a mid-infrared emission spectrometer selected for the core payload of ENVISAT by ESA due to several favourable properties (Fischer et al., 2000). Within this part of the atmospheric spectrum there is a wide variety of important molecules which have vibration-rotation bands with absorption lines well suited for detection. Atmospheric signals are generally higher than in other parts of the spectrum because the Planck function maximizes at about

Published by Copernicus Publications on behalf of the European Geosciences Union. 
$10 \mu \mathrm{m}$ for atmospheric temperatures. Generally, instruments working in the mid-infrared can be significantly smaller than those operating at longer wavelengths. This is dictated by diffraction limits and the high spectral resolution needed to observe the trace species of interest. An advantage of the mid-infrared in comparison to spectral regions with shorter wavelengths is that instruments operating in this region allow measurements during day and night.

MIPAS is a limb sounder, i.e. it scans across the horizon detecting spectral radiances emitted by the atmosphere (Fischer and Oelhaf, 1996). Due to the measurement geometry, i.e. observing the atmosphere along tangential optical paths, MIPAS can measure also trace gases with very low mixing ratios. The vertical resolution of the measured vertical profiles is in the order of some kilometres, much better compared to nadir sounding instruments. On the other hand, the limb geometry restricts the observable altitude range to heights above clouds so that information on tropospheric parameters is limited.

MIPAS can be used for scientific investigations in various research fields. A main objective is to study dynamics and chemistry of the upper troposphere, stratosphere, mesosphere, and lower thermosphere. Global measurements of many atmospheric quantities over several years contribute to climatological analyses. Weather forecasting can use the global measurements at upper atmospheric levels for data assimilation. Physical processes in the atmosphere causing e.g. non-local thermodynamic equilibrium can be studied with the highly resolved spectra much better than ever before.

In order to detect many trace gases simultaneously the instrument has to cover a wide spectral range with high spectral resolution. The concept underlying the space version of MIPAS draws on the experience gained from several experiments exploiting Fourier transform spectrometers. In particular, the MIPAS-B (balloon) experiment (Friedl-Vallon et al., 2004) can be regarded as a precursor of the MIPAS satellite experiment even if the type of interferometer is not exactly the same. An instrument similar to MIPAS-B was flown on different aircraft (Keim et al., 2004). Both types of experiment have helped to establish the feasibility of the MIPAS space experiment. Basic knowledge about interferometers gained from Fourier spectrometers measuring the attenuated solar radiation has also been taken into account. In this context the ATMOS (Atmospheric Trace Molecule Spectroscopy) experiment has to be mentioned which has yielded simultaneous measurements of a large number of trace constituents in the Middle Atmosphere (Farmer et al., 1987).

The aim of this publication is to present an overview of the MIPAS space experiment. It considers the scientific objectives and describes the instrument concept, the observing capabilities, and the calibration. A further challenge has been to fix the retrieval methods for the processing of the large amount of data in an efficient and timely fashion. This problem is addressed in Sect. 4 while Sect. 5 describes the observation strategy and the various measurement modes of MIPAS. This section includes also a short description of the functioning of the MIPAS instrument during the past years. The various measurement modes of MIPAS are to a certain degree related to the scientific objectives. Due to the absolute calibration of the measurements of a mid-infrared instrument the errors of the derived parameters can be estimated with confidence. In Sect. 7, the geophysical validation of the MIPAS measurements, which is necessary for all space experiments, is described. Some examples of MIPAS results are presented in Sect. 8 in order to demonstrate the capability of the experiment. Finally, the conclusions are summarized and an outlook to the coming years is given.

\section{Scientific objectives}

Within the earth system, the atmosphere has a central role: as an extremely efficient medium of transport it interconnects the other compartments of the earth system by cycling heat, water, and trace substances. At the same time - because of its comparatively low mass - it reacts rapidly to external forcing in particular to anthropogenic emissions.

It is well recognized that man has changed the composition of the atmosphere and the properties at the land surface which has led to changes in temperature and radiative fluxes in the atmosphere. Anthropogenic activities have also influenced the processes in the upper atmosphere (e.g. an increase of stratospheric $\mathrm{H}_{2} \mathrm{O}$ concentrations as a consequence of the tropospheric increase in $\mathrm{CH}_{4}$ ).

These global changes require global observation systems, i.e. satellite-based remote sensing experiments to measure spatial and temporal distributions of long and short-lived species, temperature, cloud parameters and radiance spectra. As stated before, MIPAS can deliver these quantities on a global scale, during day and night and over a wide altitude region.

\subsection{Stratospheric chemistry and dynamics}

Considerable progress has been made in understanding the stratospheric ozone depletion. In fact, the stratospheric ozone layer is expected to recover as a consequence of the Montreal Protocol, which demanded a reduction of ozonedepleting substances. However, considerable uncertainty about the rate of ozone recovery and future ozone levels exists, mainly due to the coupling to the growing greenhouse effect (Weatherhead and Andersen, 2006). For instance upto-date climate models do not take into account the spatial and temporal distribution of varying Polar Stratospheric Clouds when forecasting future ozone levels. Their inclusion will present a difficult task, since in polar winter the processes of stratospheric chemistry and dynamics are very complex. This was underlined in 2002, when the Antarctic 
vortex split into two parts and caused extraordinary conditions in the stratosphere.

Changes in the physical structure of the non-polar stratosphere are also taking place. Modelling studies indicate that this is partly due to the cooling of the stratosphere resulting from ozone depletion and from increasing $\mathrm{CO}_{2}$. Such changes in the basic structure of the stratosphere may well be accompanied by changes in the circulation of air within it. This in turn will change the lifetime of species in the stratosphere.

\subsection{Stratosphere-troposphere exchange}

Interactions between troposphere and stratosphere are manifold. The chemical composition of the stratosphere is largely determined by input of air from the tropical troposphere. The breakdown of the polar vortex is driven by wave disturbances from tropospheric weather systems. Mid- and high latitude tropospheric ozone depends strongly on ozone transported down from the stratosphere. Upward and downward propagating radiation fluxes are modified by the change in atmospheric composition. There is increasing evidence that stratospheric dynamics can influence tropospheric weather through the downward propagation of wave disturbances; such effects play a role in medium and long range weather forecasting.

Of particular interest are the upward transport in the Tropical Transition Layer (TTL) and the exchange processes along the tropopause breaks. Accurate measurements of long-lived trace species will help to improve our understanding of these processes.

\subsection{Chemistry and physics of the upper troposphere}

The concentration of water vapour in the upper troposphere is of great importance for the greenhouse effect due to its strong emission of radiation in the mid- and far infrared spectral region. Under high humidity conditions the water vapour emitted by aircraft causes cirrus clouds which influence the cloud cover and the radiation balance in regions of heavy air traffic. Also, the emissions of the current commercial fleet of subsonic aircraft make a significant contribution to the $\mathrm{NO}_{\mathrm{y}}$ budget in the upper troposphere.

In addition elevated concentrations of various trace gases (e.g. HCHO, acetone, PAN) are often observed in the upper troposphere indicating rapid upward transport of air from the lower troposphere and impact on ozone levels in the upper troposphere. As a consequence, characteristic patterns of trace gases in the upper troposphere point to areas of biomass burning in Africa and South America.

\subsection{Physics and chemistry of the upper atmosphere}

MIPAS has the potential to measure also many important atmospheric parameters in the mesosphere and lower thermosphere (MLT), namely temperature, carbon dioxide, carbon monoxide, ozone, water vapour, methane and nitric oxide. These observations contribute to improve our understanding of major research areas of the middle and upper atmosphere: the temperature structure and energy balance, the chemistry, the dynamics, and the interaction between them. In addition, the quality of MIPAS data allows the study of the nonlocal thermodynamic equilibrium (Non-LTE) of the middle and upper atmosphere. This knowledge is crucial when the species are retrieved from their Non-LTE IR emission and hence helps in retrieving more accurate concentrations from wideband IR radiometers.

A very comprehensive review of the middle and upper atmosphere has been compiled by Siskind et al. (2000). This monograph provides an insight into and an update of our current knowledge of this atmospheric region. The four major areas described there are energetics, chemistry, dynamics, and long term changes. In case of energetics, MIPAS measures the most important emissions contributing to the IR radiative cooling of the upper atmosphere $\left(\mathrm{CO}_{2}, \mathrm{NO}\right)$. MIPAS also measures the most significant chemical compounds of this region, including $\mathrm{O}_{3}, \mathrm{H}_{2} \mathrm{O}, \mathrm{CH}_{4}, \mathrm{CO}$, and $\mathrm{CO}_{2} . \mathrm{O}_{3}$ is very much affected by tides (Smith and Marsh, 2005) and hence might help in understanding the chemistry/dynamics interactions. Water vapour plays a major role in the upper atmospheric chemistry. It has been found to increase in the middle atmosphere during 1992-1996 (Nedoluha et al., 2000). Therefore it is important to discern if this change continues or regresses. Water vapour has been scarcely measured in the middle atmosphere, particularly in the cold summer mesopause.

Carbon monoxide and carbon dioxide yield the total carbon budget in the upper atmosphere. The distribution of these trace gases is significant for understanding the interplay between molecular diffusion and vertical eddy diffusion in the upper mesosphere and lower thermosphere (LópezPuertas et al., 2001). Carbon monoxide will also provide important information on the dynamics of the mesosphere, e.g., the meridional circulation at solstice and the transition from equinox to solstice conditions and vice versa (see, e.g. López-Valverde et al., 1996). Another essential topic is the dynamics of the upper part of the polar vortex, such as the downward motion of air masses from the mesosphere into the stratosphere during the polar night, for which $\mathrm{CO}$ is an excellent tracer.

The middle atmosphere is also influenced by the solar electromagnetic radiation and particle flux. High solar activity is changing many trace gas concentrations in the atmosphere; it even causes a decrease in the ozone amount of the stratosphere.

\subsection{Climatology and weather forecasting}

Chemistry-climate interaction in the stratosphere is among other things due to the role that ozone has in determining the stratospheric temperature. In this context it is important 


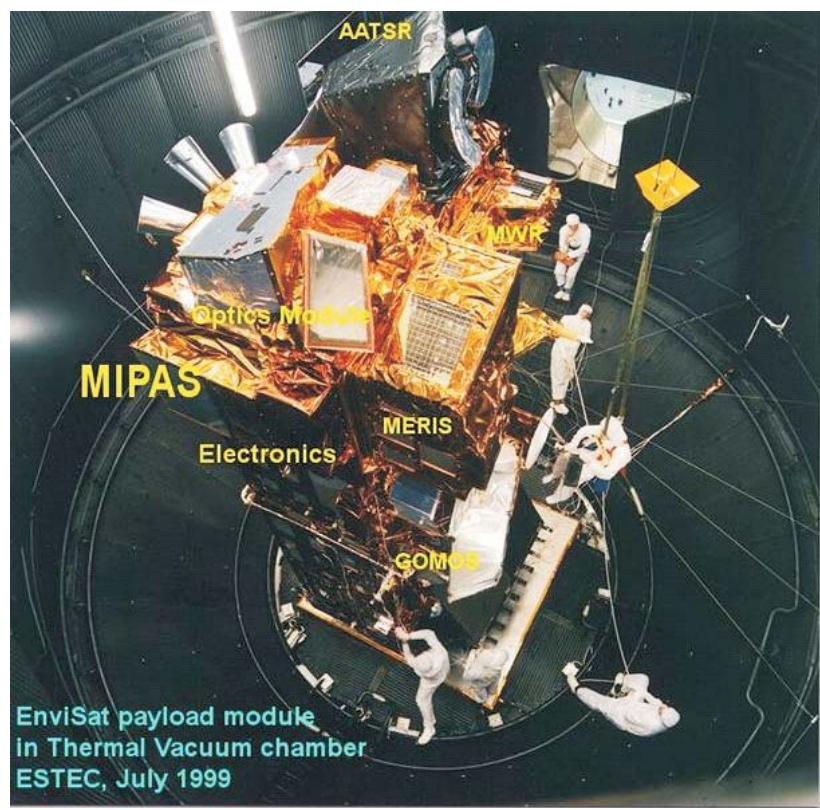

Fig. 1. The MIPAS instrument on top of ENVISAT surrounded by other instruments (AATSR, MWR, MERIS); at the top the optics module with the two baffles is recognized, below the electronics module is fixed on the satellite structure (provided by ESA).

to study the year to year variation in the abundances of $\mathrm{Cl}_{\mathrm{y}}$, $\mathrm{HO}_{\mathrm{y}}$, and $\mathrm{NO}_{\mathrm{y}}$ as they may provide insights into the causes of variations of ozone levels. MIPAS is able to provide extensive observations of water vapour, both in the stratosphere and upper troposphere, which will be significant in further understanding of the cooling of the atmosphere. Another important contribution that MIPAS could make to climatology is to provide observations of the global distribution of thin cirrus clouds and Polar Stratospheric Clouds (PSCs). The coverage and the optical properties of these clouds are not well known, resulting in an uncertainty in their contribution to the radiation budget.

The trends in the concentrations of trace constituents are small and over a period of five years will probably be too small to be unambiguously detected by a limb sounding experiment. For this purpose the same instrument would have to be flown several times sequentially. Another possibility is to combine the MIPAS data with other satellite or with long term ground-based measurements.

Today's operational meteorological satellites deliver profiles of temperature and water vapour with limited vertical resolution and accuracy in the tropopause region and above. Information on ozone is restricted to the vertical column amount.

It has already been shown that the higher vertical resolution of MIPAS temperature and ozone measurements in the upper troposphere/lower stratosphere has a positive influence on the data assimilation of the weather forecast (Dethof,
2003). This means, ECMWF is interested to obtain near real time MIPAS data on a regular basis for its weather forecast.

\subsection{Further scientific areas}

In recent years the chemical weather forecast and the forecast of ozone fields have been developed. The operation of the numerical models requires data assimilation as in case of the weather forecast. Consequently, there is a great demand for data from global measurements of trace constituents in order to improve the initial conditions for the model runs.

\section{The instrument}

\subsection{Instrument concept}

\subsubsection{General remarks}

MIPAS is a Fourier transform infrared (FTIR) spectrometer aboard ENVISAT (Fischer et al., 2000, Fig. 1) for the detection of limb emission spectra in the middle and upper atmosphere. It observes a wide spectral interval ranging from $4.15 \mu \mathrm{m}$ to $14.6 \mu \mathrm{m}$ with high spectral resolution. The measured data set is very rich in information as each spectrum comprises of the order of 50000 spectral elements (Fig. 2). The primary geophysical parameters of interest are vertical profiles of atmospheric pressure, temperature, and volume mixing ratios (vmrs) of at least 25 trace constituents.

The advantage of a FTIR instrument over spectrometers with dispersive elements like gratings or prisms lies in the fact that a single detection element can be used to record a broadband spectrum with high spectral resolution while for dispersive spectrometers either a scanning detector must be used, or a large array detector must be employed which simply did not exist in the mid-infrared at the time of constructing MIPAS. Thus a FTIR was the only way to obtain complete high resolution IR-spectra with good sensitivity.

The drawback of a FTIR is its mechanical complexity, comprising moving optical elements which have to be guided with a very high precision over an extended distance, and very high alignment stability required for all optical components in the interferometer. In the case of MIPAS, the alignment constraints become even more challenging as the optics is cooled down to $210 \mathrm{~K}$ by a large radiator in order to reduce the thermal radiation background of the instrument.

\subsubsection{MIPAS functional design}

As schematically shown in Figs. 3 and 4, the atmospheric radiance enters the instrument through the Front End Optics where it is reflected at an azimuth and an elevation scan mirror. Afterwards the IR beam traverses an off-axis anamorphotic telescope which matches the beam size to the input aperture of the interferometer and enters a dual slide 


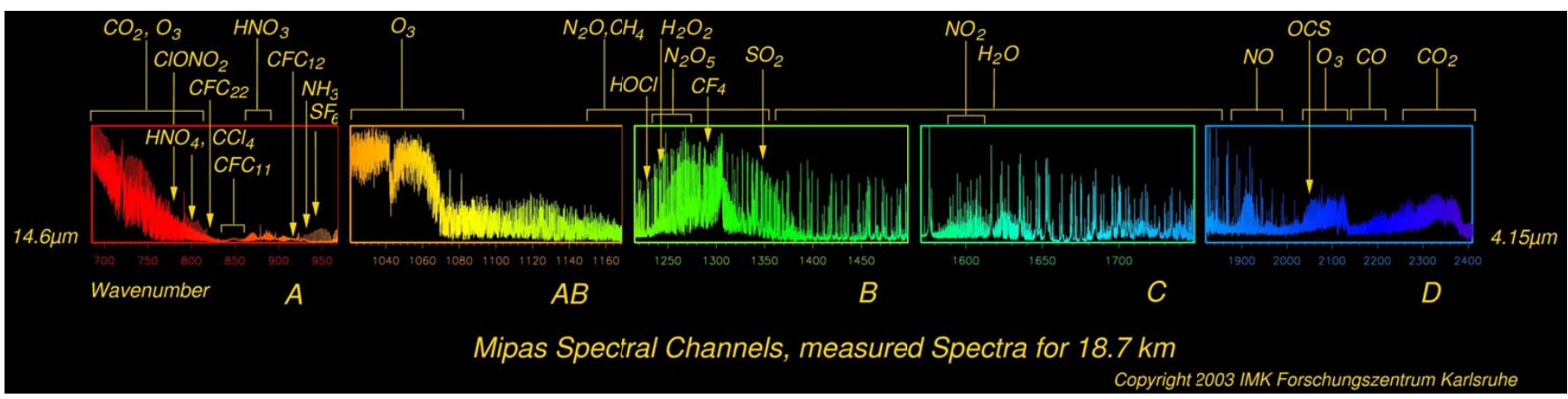

Fig. 2. The total spectral region $(4.15-14.6 \mu \mathrm{m})$ of MIPAS as measured at $18.7 \mathrm{~km}$ tangent altitude, is divided in 5 spectral intervals $(\mathrm{A}, \mathrm{AB}$, $\mathrm{B}, \mathrm{C}, \mathrm{D})$; the large number of spectral emission lines demonstrates the enormous amount of information in the measurements; a considerable number of trace constituents can be detected as indicated.

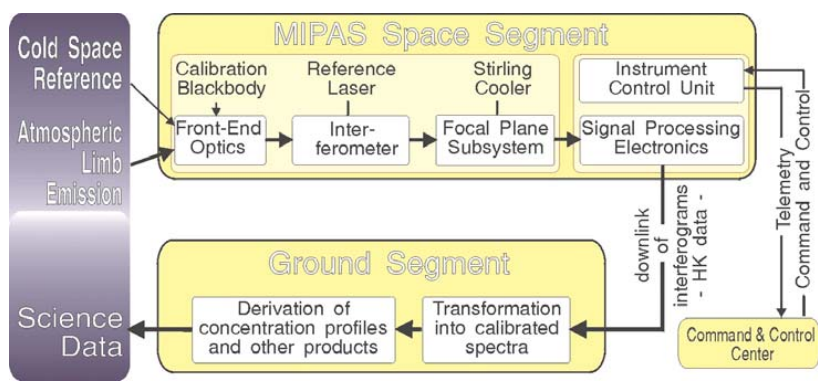

Fig. 3. MIPAS Signal Flow (provided by ESA).

Michelson-type Interferometer with two input and two output ports. The second input port receives as little IR radiation as possible from the cold focal plane inner structure. Both input signals are divided at the beamsplitter inside the interferometer and directed to two movable corner cubes from where they are reflected to the beam recombiner; the recombined intensity modulated signal constitutes the interferogram and appears at both output ports.

As the interferogram mathematically corresponds to the Fourier transform of the measured spectrum, a highly accurate sampling allows to derive on ground the originally measured spectrum of the modulated signal via an inverse Fourier transform. The sampling is provided by a reference laser with a sampling frequency of $7612 \mathrm{~cm}^{-1}$ and is also used to achieve a constant speed of the cube corners.

The output signal at each port enters the Focal Plane Subsystem which is actively cooled by a pair of low vibration Stirling-cycle coolers to $70 \mathrm{~K}$ for maximum sensitivity of the detectors. Inside this subsystem each input signal is separated into four spectral channels by optical filtering and analysed by dedicated MCT (Mercury Cadmium Telluride) detectors.

After pre-amplification, the eight signals enter the analogue part of the Signal Processing Electronics (SPE) where the DC-signals are suppressed by AC-coupling. The ACsignals are amplified, lowpass filtered to avoid aliasing and

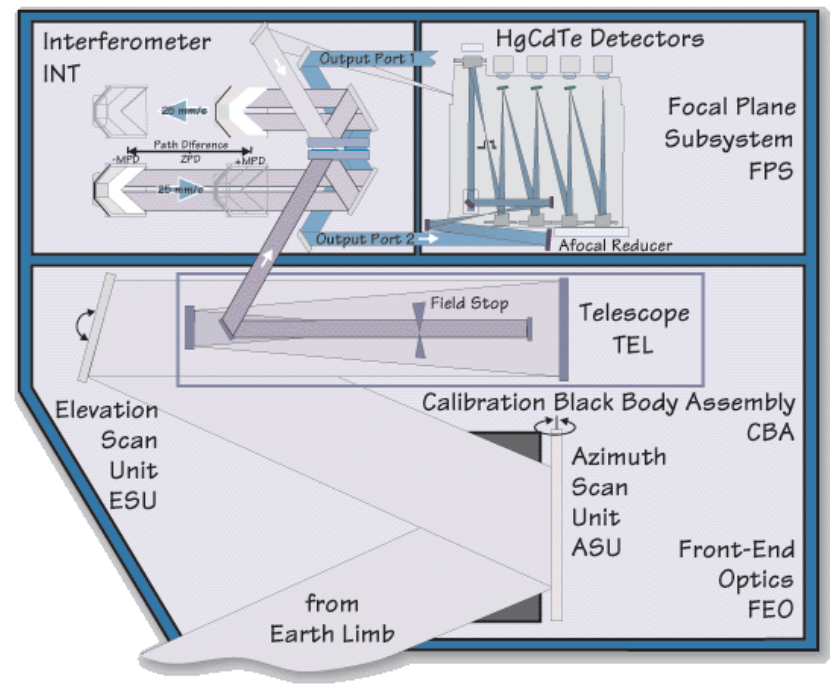

Fig. 4. MIPAS Optical Layout (provided by ESA).

then $\mathrm{A} / \mathrm{D}$ converted with 16 bit resolution.

The digital part of the SPE separates in each band the spectral range of interest by complex filtering. In a further processing step measurement data are multiplexed and formatted to source packets which include auxiliary data for ground processing purposes.

A redundant Instrument Control Unit provides the telemetry/telecommand interface to the platform and monitors autonomously the instrument status.

In the ground segment, the down-linked interferograms are converted into calibrated atmospheric spectra which are further used to retrieve the concentration profiles of the relevant atmospheric species and other higher level products.

\subsubsection{Functional description of the optics module}

The MIPAS optics module is installed at the anti-sunward side of ENVISAT and has the following major parts: Front End Optics, Interferometer and Focal Plane Subsystem. 


\section{Front End Optics}

The Front End Optics consists of Azimuth Scan Unit, Elevation Scan Unit, telescope, and calibration blackbody. The individual items are briefly described in the following:

The Azimuth Scan Unit (ASU) allows the selection of the line of sight within the two field-of-view regions, and also enables the detectors to view an internal calibration blackbody source for gain calibration. A flat steering mirror is rotated about an axis parallel to nadir to direct the light into the instrument. This steering mirror has a dimension of about $295 \mathrm{~mm}$ in height and $109 \mathrm{~mm}$ in width and thus forms the largest optical component of MIPAS.

A second function of the ASU is the protection of the interior of the optics module from contamination; a shield is mounted behind the steering mirror and rotates with it. When the mirror is turned to an end stop, the shield closes the input aperture to the ASU and thus the ASU mirror is protected from contamination during ground handling and in the early flight phase.

The Elevation Scan Unit (ESU) determines the actual limb height of a particular measurement, and thus requires a very high pointing accuracy over a limited angular range. It comprises a flat steering mirror rotating around an axis that is orthogonal to nadir and flight direction. The angle covered by this mirror is less than $3^{\circ}$ which is sufficient to reach tangent heights between $5 \mathrm{~km}$ and $250 \mathrm{~km}$; the high value is used for measurements of cold space to determine the instrument self-emission for offset calibration.

After being reflected by the Elevation Scan Unit the beam enters the anamorphotic telescope which reduces its dimensions to $25 \mathrm{~mm}$ by $50 \mathrm{~mm}$. This telescope also houses the field stop that determines the field-of-view of the instrument. The position of the field stop in front of the interferometer ensures that all detectors view the same air volume.

Mounted in the azimuth scan unit is the calibration blackbody (CBB), used for the in-flight calibration of the instrument responsivity. To fill the IFOV (Instantaneous Field-of-View), it needs a rather large clear aperture (about $165 \times 55 \mathrm{~mm}^{2}$ ). Its design is derived from the blackbody design for the along-track scanning radiometer (ATSR), flying on the ERS- 1 and -2 satellites. Its emissivity is above $99.6 \%$, so that a high accuracy for the gain calibration becomes achievable. For precision gain calibration measurements, it can be heated to about $40 \mathrm{~K}$ above the ambient instrument temperature to increase its radiance emission. Its nominal temperature then reaches up to $250 \mathrm{~K}$.

\section{Interferometer}

A symmetrical dual slide interferometer with dual input and dual output ports has been selected in order to meet the radiometric and spectrometric performance requirements, as well as the lifetime requirement of five years of continuous operation in space. This kind of interferometer provides highest detectable signal at the outputs, the least uncertainties in design, the highest degree of redundancy, and the most compact dimensions. It has a folded path to allow for a more compact arrangement of the interferometer and to allow for a better compensation of the momentum generated by the corner cubes during the reversal of their motion. The incident angle of the radiation onto the beamsplitter is $30^{\circ}$ to reduce polarization effects by the beamsplitter. The MIPAS interferometer is $0.58 \mathrm{~m}$ long and about $0.36 \mathrm{~m}$ wide, and has a mass of about $30 \mathrm{~kg}$. It has the following major subassemblies: interferometer optics, drive units, and optical path difference sensor.

The interferometer optics comprises the beamsplitter assembly, flat steering mirrors, and the corner cubes on the slides. The beamsplitter coatings themselves are quite critical, as they have to provide a reflectivity near 50\% throughout the broad spectral range. More difficult to manufacture are the broadband antireflection coatings on the other surfaces that are essential to reduce undesired interferometer effects that would modulate the transmission of the substrate and could result in ghost spectra. The beamsplitter assembly also has to compensate for the phase delays caused by the varying refractive index throughout the spectral range. This is done with a second substrate of the same thickness as the beamsplitter itself and mounted with a narrow gap to the beamsplitter coatings. Both substrates are slightly wedged to reduce the residual etalon effects.

The two identical Interferometer Drive Units perform the actual translation of the corner cubes. Linear motors behind the corner cubes generate the drive force. The slides are guided by mechanical bearings. Lifetime tests of the bearings have shown that dry-lubricated ballbearings operating with a light preload can well achieve 20 million motion cycles (in the meantime the interferometer has performed considerably more motion cycles). The difference velocity between the two slides has to be controlled with less than $1 \%$ rms error. A drive control loop processes the inputs from linear optical encoders in each of the drive arms for a coarse control and for centering of the slides, and from a built-in laser interferometer (called the optical-path difference sensor ODS) for fine velocity control. The laser interferometer is also required to trigger the sampling of the detector output at very precise intervals of optical path values.

The built-in laser interferometer makes use of a singlemode 1.3 micron diode laser which is located in the optics module near the Stirling coolers. The output of the diode laser is guided by a single mode polarizing optical fibre to the interferometer. Although the individual components had been proven in many communication systems, their use in a spaceborne instrument with operation over a wide temperature range is new and required space qualification. 
Focal plane subsystem

The two output beams of the interferometer are reduced in size by two small off-axis Newtonian telescopes, and directed into the cold focal plane subsystem, which houses the signal detectors with their interfaces to the active coolers, as well as the associated optics required for spectral separation and beam shaping. It is smaller than the interferometer $(0.36 \mathrm{~m}$ wide, $0.45 \mathrm{~m}$ high $)$ and has a mass of $16 \mathrm{~kg}$. The focal plane subsystem has the following elements: detector/preamplifier unit and focal-plane cooler assembly.

To achieve the best radiometric sensitivity, a set of four detectors in each output port is used, each optimized for highest sensitivity in a spectral band. A set of dichroic beamsplitters and steering mirrors separates the input from the two interferometer ports to the different spectral bands, and the optics required to illuminate each detector element. All optical elements are mounted and aligned in a very tight package. All optics and the detectors are cooled to about $70 \mathrm{~K}$ to reduce their thermal emission and internal noise contribution. Cooling is performed by a pair of active Stirling cycle coolers. Thus, although the focal plane subsystem is conceptually a simple design, the numerous interfaces between the optics, the detectors, and the coolers under the constraints of good thermal insulation and high alignment stability of the optical components result in very demanding requirements.

An analysis for the optimization of the detectors has shown that four spectral bands (A, B, C, D) in each interferometer output port are required to achieve the low instrument noise contribution and to provide some redundancy at the long wavelength region for temperature measurements. Thus a total of eight detector elements are needed in MIPAS. In the long wave spectral region (14.6 to about $7 \mu \mathrm{m}$ ), only photoconductive $\mathrm{HgCdTe}$ detectors (PC-MCT) were able to meet the specifications on low noise contribution and electronics bandwidth when the instrument was built. At the shorter wavelengths (7 to $4 \mu \mathrm{m}$ ), photovoltaic $\mathrm{HgCdTe}$ detectors (PV-MCT) are the best choice. The preamplifiers are individually optimized for each detector to fulfill stringent requirements on noise, phase distortions and linearity. The cold part of the preamplifiers is mounted in the detector housing, while final amplification is performed in an externally mounted package at ambient temperature. Stirling cycle coolers with a performance that satisfies the cooling requirements of MIPAS $(500 \mathrm{~mW}$ heat lift at $70 \mathrm{~K}$ temperature) are used in a twin-cooler arrangement, comprising two identical compressor and displacer units that operate synchronously to compensate most vibrations from the oscillating parts.

\subsubsection{Further Subsystems}

Further subsystems are the Signal Processing Electronics and the Instrument Control Unit.
The Signal Processing Electronics (SPE) takes the analogue signal inputs from the detector preamplifier unit and generates the associated digital bit streams, which are then transformed into formatted data packets and sent to the platform bus for transmission to ground. These data are accompanied by the requisite auxiliary data from the SPE \& ICE (Instrument Control Electronics) needed to interpret and process the instrument measurements. In order to satisfy the downlink bandwidth allocated to the instrument, the SPE must perform certain digital operations upon the bit streams.

The SPE processing can basically be divided into the analogue signal processing (ASP), the digital signal processing (DSP), and the output processing (OP). In the analogue signal processing the signals are, after input amplification, low pass filtered to eliminate aliasing in the sampling process and then A/D converted. Nominal sampling frequency at the SPE is approximately $77 \mathrm{kHz}$ in each channel, with the spectrum of interest lying in the range 6.85 to $24.1 \mathrm{kHz}$. For the LOS calibration mode, data are received only on the shorter wavelength (D1 and D2 for the two output ports) channels and are sampled at around $100 \mathrm{~Hz}$ using an SPE-generated clock signal.

The digital signal processing of the SPE isolates the five nominal bands (generated by a combination of the 2 times 4 filter ranges, see Kleinert et al., 2007) through a digital filtering operation and performs a channel addition to improve SNR. The SPE performs additional digital operations to reduce the output data rate to the required level. Following digitisation, the SPE records the maximum and minimum digital value recorded on each channel of each interferogram. Each channel is then digitally filtered to isolate the band of interest and the data size is reduced by a sub-sampling operation. The final operation in this chain is the truncation of the data words to be sent to the Data Handling Assembly of the ENVISAT satellite. In a typical interferogram, only the very central portion (around 1\%) contains significant values and the data outside require considerably less bits for accurate representation. The most significant bits which are set to zero can therefore be discarded without loss of information.

The Output Processor generates source packets in each of five bands and then multiplexes the source packets into the output stream to the Data Handling Assembly.

The controlling functions of the MIPAS instrument are performed by the Instrument Control Unit, which runs the MIPAS specific application software. The functions of the Instrument Control Unit comprise among others the control of the MIPAS operation and its operational modes, recording of instrument events, commanding and monitoring of MIPAS equipments, monitoring and processing of MIPAS house keeping data, and initiation of corrective actions in case of instrument failures. 


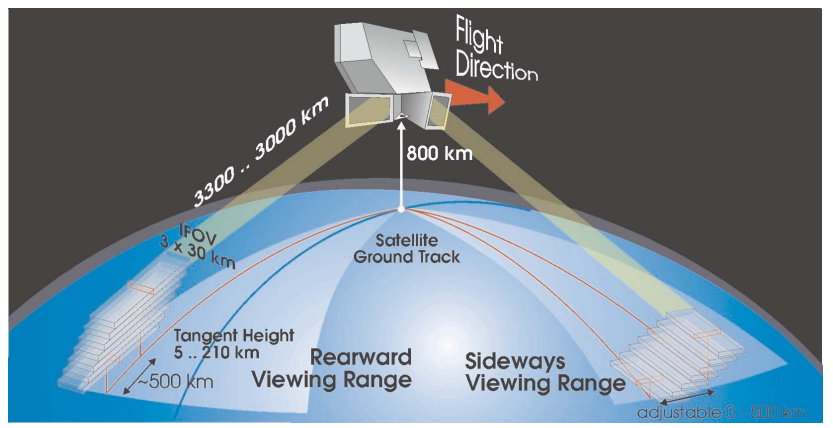

Fig. 5. MIPAS Observation Geometry (provided by ESA).

\subsection{Observing capability}

MIPAS observes the emitted radiance from the atmosphere at the limb, i.e. the line of sight penetrates the atmosphere down to a minimum altitude, named the tangent altitude, and then, because of the Earth's curvature emerges again towards space. This type of observation is selective to the atmospheric signal emitted from the tangent altitude layer and, when a limb sequence with a discrete set of different tangent altitudes is acquired, allows the determination of the vertical profiles of atmospheric parameters. In broad terms MIPAS can observe atmospheric parameters in the altitude range from 5 to $160 \mathrm{~km}$ with minimum and maximum steps of 1 and $8 \mathrm{~km}$, respectively.

To provide access to any air volume in the atmosphere the MIPAS pointing system uses two mirrors. The elevation mirror selects the limb altitude and corrects for platform attitude, orbital altitude and Earth's geoid geometry. The azimuth mirror determines the location of the observed air volume relative to the platform position. The azimuth mirror provides access to limb targets rearwards within a $35^{\circ}$ wide range around the anti-flight direction and sideways within a $30^{\circ}$ wide range on the anti-Sun side of the satellite (Fig. 5).

For operational measurements MIPAS performs observations in the rearward viewing direction. In this case the sampling strategy is to keep the azimuth mirror fixed during each limb scanning sequence. The limb sequence is performed by changing the orientation of the elevation mirror with discrete steps in tangent altitude. At each nominal position of the elevation mirror an interferometer sweep is acquired while the elevation mirror is actively controlled in order to maintain a constant tangent altitude. As the tangent altitude is decreasing its location moves away from the platform. For this reason the limb sequence is performed scanning down from high altitudes to low altitudes, so that the geometrical effect partially compensates for the movement of the platform and an almost vertical sequence of tangent altitude points is obtained. By changing the azimuth angle with respect to the rearward direction it is possible to move the tangent altitude location away from the satellite ground track. Since the ground track is limited to the latitude region between about $80^{\circ} \mathrm{S}$ and $80^{\circ} \mathrm{N}$ (due to the inclination of the orbit) the azimuth angle is occasionally changed during the orbit in order to perform observations from pole to pole.

For cross track observations, simultaneous changes of azimuth and elevation angle between subsequent sweeps can be considered in order to compensate for the platform movement.

The time required to record one full resolution spectrum is about $4.5 \mathrm{~s}$. A nominal limb scanning sequence consists of 17 spectra, measured at tangent altitudes from 6 to $68 \mathrm{~km}$ with a spacing of $3 \mathrm{~km}$ at low altitudes and larger spacing above, and is acquired in a total time of about $76 \mathrm{~s}$. During each orbit about 72 limb sequences are acquired as well as calibration measurements. Each day about 14 orbits are obtained for a total of about 1000 limb sequences or profiles per day.

Important features of the MIPAS observation strategy are:

- emission measurements with continuous observations independently of the presence of external radiation sources and of the day/night conditions,

- limb sounding technique with good resolution of the vertical distribution of the atmospheric parameters,

- polar orbit with azimuth correction for global (pole to pole) mapping,

- broadband FTS measurements with simultaneous observation of an almost complete survey of the atmospheric constituents.

The combination of these features makes it possible to obtain a comprehensive picture of the atmospheric chemistry with daily three-dimensional (altitude-latitude-longitude) global maps of the concentration of a considerable number of constituents for the full duration of the instrument mission. The flexible altitude steps of the elevation mirror allow the vertical sampling of the parameter profiles to be increased in atmospheric regions where the gradient in concentration is changing rapidly (e.g. tropopause, denitrificated layers in the stratosphere). No other satellite instrument has ever combined all these features for the investigation of middle atmosphere phenomena (see Sect. 8).

\subsection{Calibration and characterization}

A detailed description of the calibration and characterization of the instrument is given by Kleinert et al. (2007). Therefore, only the most important processing steps and results are summarized here.

\subsubsection{NESR}

The NESR (noise equivalent spectral radiance) quantifies the noise level of the data. It ranges from $3 \mathrm{nW} /\left(\mathrm{cm}^{2} \mathrm{srcm}^{-1}\right)$ to $50 \mathrm{nW} /\left(\mathrm{cm}^{2} \mathrm{srcm}^{-1}\right)$, depending on the spectral range and channel. The two times four detectors comprise the spectral 


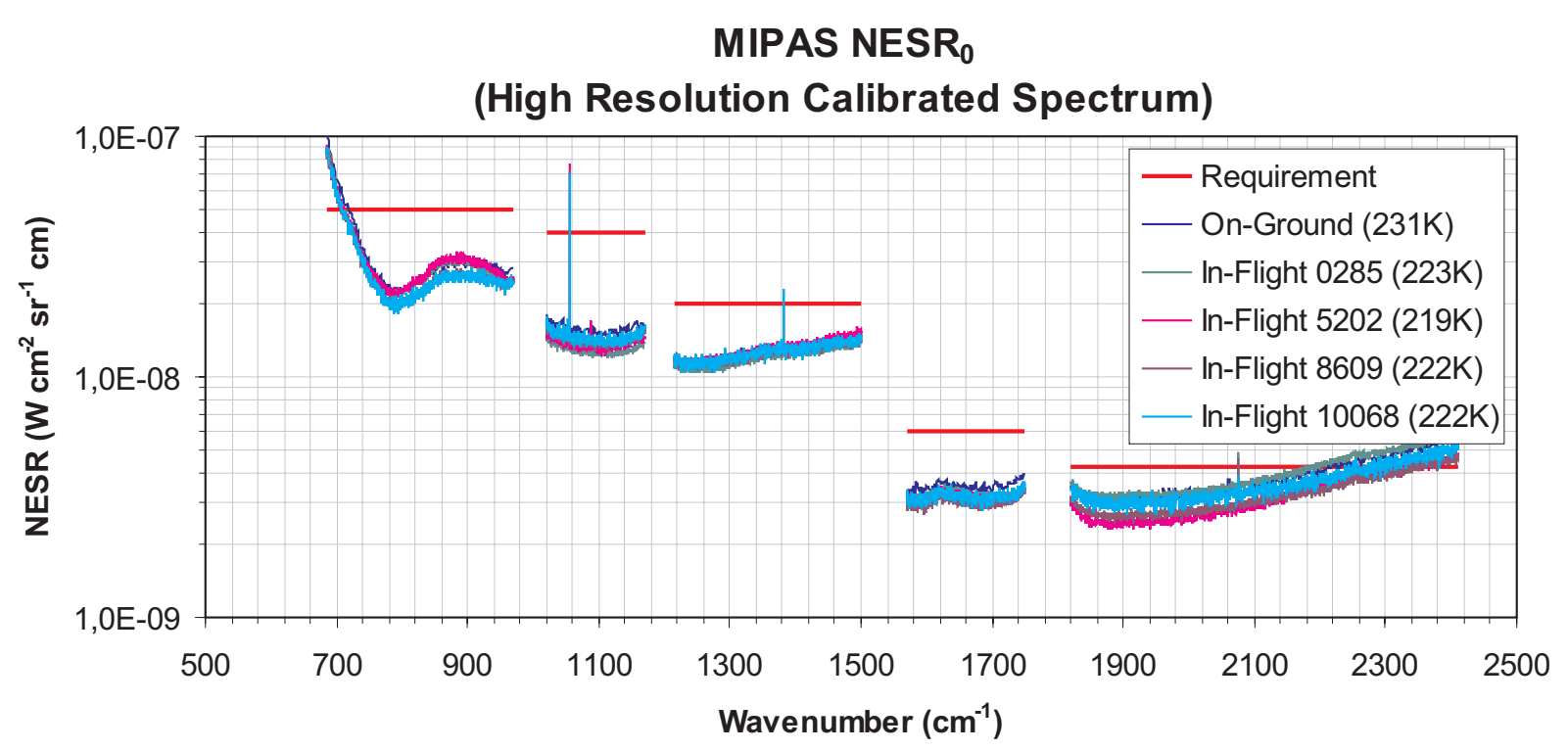

Fig. 6. NESR 0 of MIPAS on ground and in flight. The index 0 denotes the absence of radiation from the scene.

range from 685 to $2410 \mathrm{~cm}^{-1}$ which is split into five bands $\mathrm{A}$, $\mathrm{AB}, \mathrm{B}, \mathrm{C}$, and D, each band being covered by one or two specific detectors (details see Kleinert et al., 2007). The NESR is well within the requirements (Fig. 6), and no systematic evolution of the NESR over time could be observed. The most important effect on the NESR is ice accumulation on the detector system, which may temporarily degrade the NESR by up to $20 \%$ (value depends on the frequency of decontamination) with respect to an ice free detector.

\subsubsection{Radiometric calibration}

In order to assign absolute radiance units to the measured spectra, gain and offset measurements are performed regularly. The instrument offset is determined by looking into deep space, and measurements of a blackbody with known temperature are performed to determine the gain function. In principle, the gain function $G$ is calculated as:

$G=\frac{L_{B B}}{S_{B B}-S_{D S}}$

where $S_{B B}$ and $S_{D S}$ are the measured blackbody and deepspace spectra, respectively, and $L_{B B}$ is the spectral radiance of the blackbody. The atmospheric spectra are then calibrated using:

$L_{\mathrm{atm}}=G\left(S_{\mathrm{atm}}-S_{D S}\right)$

with $S_{\text {atm }}$ and $L_{\text {atm }}$ being the measured and calibrated spectrum, respectively.

\subsubsection{Gain and offset stability}

The gain function is much more stable than the instrument offset. Therefore, it is usually sufficient to generate a gain calibration function once per week while the offset is measured several times per orbit (about every $7 \mathrm{~min}$ ). The radiometric gain stability is typically better than $1.5 \%$ per week, and the offset change between two consecutive measurements is below $10 \%$ of the NESR. One reason for gain drift is the formation of ice on the cooled detector optical components which especially affects regions with strong infrared absorption bands of ice.

\subsubsection{Detector non-linearity}

The photoconductive detectors from the long wavelength channels $\mathrm{A}, \mathrm{AB}$, and $\mathrm{B}$ are subject to non-linearity depending on the total incident photon flux. This non-linearity needs to be corrected before the radiometric calibration Formulas (1) and (2) are applied. The non-linearity is corrected by scaling each interferogram with an appropriate factor which is calculated from the non-linearity parameters and the peak-to-peak value of the interferogram, which is a measure for the total photon flux. The non-linear behaviour of the detectors has been characterized and parameterized on-ground and crosschecked in-flight using commissioning phase data. Based on the intercomparison of the on-ground and in-flight data, the accuracy in the calibrated scene spectra with respect to the non-linearity correction is estimated to be within a few percent, depending on the line intensities and the total photon flux. The error depends on instrument temperature, ice layer thickness on the detector optics, detector temperature, and detector aging. 


\subsubsection{Spectral calibration}

Spectral calibration is performed using known features of standard limb measurements. Appropriate spectral lines with well known peak positions are used in each spectral band to determine a spectral shift in the measured spectrum. For the shift determination, four spectra of a tangent altitude of $32 \mathrm{~km}$ are co-added. The wavenumber dependence of the spectral shift is in first approximation assumed to be linear but a second order effect is also considered in the level 2 analysis (see Raspollini et al., 2006, this special issue). The spectral shift is assumed to be the same for all types of measurements and is applied until a new calibration is performed. To ensure a stability and an accuracy better than $0.001 \mathrm{~cm}^{-1}$, the spectral calibration (shift determination) is performed every four limb sequences, i.e. every $320 \mathrm{~s}$. The spectral shift has not changed significantly during the mission; its variation from July 2002 to March 2004 is in the order of 6 ppm.

\subsubsection{Instrumental Line Shape}

The instrumental line shape (ILS) is the area-normalized spectral response function of the spectrometer. When a monochromatic spectral line at position $\sigma_{0}$ is irradiated, the ordinate value of the area-normalized response at $\sigma$ is $\operatorname{ILS}\left(\sigma-\sigma_{0}, \sigma_{0}\right)$. Due to the normalization, the specification of an ILS is not affected by the calibration of the radiance axis. In contrast, there is an intimate connection with spectral shift, however, the spectral shift may be treated separately (see Sect. 3.3.5).

The ILS of MIPAS is specified by means of a set of ILS variables. Because a physical model of the interferometer is available for MIPAS, the subset of model variables which affect the ILS can be adjusted to approximate the ILS of the real instrument. The suggested parameterization has been used in various studies. It is found that the loss in peak response of the unapodized ILS due to misalignment amounts $2 \%$ at $800 \mathrm{~cm}^{-1}, 5 \%$ at $1200 \mathrm{~cm}^{-1}, 8 \%$ at $1600 \mathrm{~cm}^{-1}$, and $11 \%$ at $2000 \mathrm{~cm}^{-1}$ when the instrument is operated in full nominal resolution.

\subsubsection{Field-Of-View characterization}

Analyses of the instrument's field-of-view (FOV) response and the co-alignment of the various detector channels were performed both prior to launch and post launch. The prelaunch characterization of the FOV width, based on optical measurements, yielded a value of 52 mdeg (FWHM) in the elevation direction. Verification measurements were conducted at IR wavelengths for individual detector channels, with the instrument collimated input beam viewing a hot blackbody source equipped with a small circular aperture. Additional verification measurements were conducted in flight. These were based on the active scanning of the instrument's line of sight across an infrared bright, "point like" source while raw mode interferograms were acquired in all eight detector channels.

Results of both types of verification measurements were found to be consistent with the optical characterization data, taking into account inaccuracies inherent to the experimental set-up in each case. From in-flight measurement the coalignment of the FOV patterns was verified to be better than 1.3 mdeg in elevation for all detector channels.

\section{Retrieval methods for MIPAS}

\subsection{The operational code for MIPAS data processing}

While the level 1 analysis (see Sect. 3) determines the geolocated and calibrated spectra from the raw instrument data, the level 2 analysis performs the retrieval of the geophysical parameters of interest from level $1 \mathrm{~b}$ spectra. In the operational level 2 analysis the pressure at tangent altitudes (denoted as tangent pressure) and the vertical profiles of temperature and six species selected to have highest priority, namely $\mathrm{O}_{3}, \mathrm{H}_{2} \mathrm{O}$, $\mathrm{CH}_{4}, \mathrm{HNO}_{3}, \mathrm{~N}_{2} \mathrm{O}$, and $\mathrm{NO}_{2}$, are retrieved. These profiles are the targets of the operational analysis and are retrieved as a function of tangent pressure. Other minor atmospheric constituents are retrieved with non-operational scientific codes (see Sect. 4.2).

All retrievals are based on the non-linear least-squares fit (Menke, 1984; Sivia, 1998; Rodgers, 2000) of a parametrized forward model to the measured spectra. A theoretical forward model calculation $\boldsymbol{F}(\boldsymbol{p}, \boldsymbol{x})$ is fitted to the observations $\boldsymbol{y} . \boldsymbol{F}(\boldsymbol{p}, \boldsymbol{x})$ simulates the observations starting from the quantities $\boldsymbol{x}$ to be retrieved and from a set of instrumental and geophysical parameters $\boldsymbol{p}$, that are assumed to be known. The solution is found with an iterative procedure that uses the Gauss-Newton method for the minimisation of a cost function. Usually, the cost function is the $\chi^{2}$ function, equal to:

$\chi^{2}=\boldsymbol{n}_{\text {iter }}^{T} \boldsymbol{V}_{n}^{-1} \boldsymbol{n}_{\text {iter }}$,

where $\boldsymbol{n}_{\text {iter }}=\boldsymbol{y}-\boldsymbol{F}\left(\boldsymbol{p}, \boldsymbol{x}_{\text {iter }}\right)$ is the vector of the residuals at iteration iter, with $\boldsymbol{x}_{\text {iter }}$ equal to either the initial guess or the result of the current iteration, and $\boldsymbol{V}_{n}$ is the Covariance Matrix of the observations.

An important feature of the MIPAS data inversion problem is that the limb sounding technique provides a selective information on the atmospheric composition at the tangent altitudes which, combined with the redundant information of the spectrally resolved measurements, leads to an usually well conditioned inversion problem even if made rather elaborate by the large number of available observations. The different retrieval codes differ in the adopted degree of sophistication and optimization of the forward model calculation, for the choice of the cost function and in the selection of the fitted observations. 
The operational MIPAS level 2 data processor was conceived by an international consortium which developed the scientific prototype, named Optimized Retrieval Model (ORM, Ridolfi et al., 2000), used for the operational implementation. The main challenge of the ORM has been the requirement of performing the complex operation of mathematical inversion in near real time (NRT) for a large number of data. The ORM code is also the prototype of the OffLine (OL) level 2 processor that is used for the re-analysis of the measurements with improved geolocation and set up of the level 1 and level 2 chain. In the off-line analysis the NRT requirement does not apply any longer, but the continuous emission measurements of MIPAS still require that the computing time is significantly shorter than the measurement time.

The main features of the level 2 operational code and the possible differences with other scientific codes are briefly recalled. For a more detailed description of the ORM and of its performances we refer to Raspollini et al. (2006).

The ORM performs a non-linear least squares fit with a cost function modified according to the LevenbergMarquardt (Levenberg, 1944) method.

The choice of the fitted observations is a compromise between the maximum information and the minimum complexity of the retrieval from the point of view of number of unknowns and observations. For this purpose, on one hand the global fit approach (Carlotti, 1988) is adopted, with the simultaneous fit of the spectra of the full limb sequence for the retrieval of each vertical profile. The global fit provides a full exploitation of the measurements and a rigorous determination of the correlation between atmospheric parameters at the different altitudes. On the other hand, apart from pressure and temperature that are simultaneously determined, the different target species are individually retrieved with a sequence that follows an order determined by the degree of spectral interference. Furthermore, the retrieval is performed using a set of narrow (less than $3 \mathrm{~cm}^{-1}$ width) spectral intervals, called "microwindows" that are selected as those intervals that contain the best information on the target parameters and are less affected by systematic errors.

The forward model calculations are also the result of a compromise between accuracy and computing time. The radiative transfer model calculates the atmospheric emission spectrum taking properly into account the effect of refractive index in the optical path, the Voigt profile of the atmospheric line shape, and using a high resolution wavenumber grid for the calculation of the monochromatic spectral radiances. The forward model determines the observed spectrum by convolving the monochromatic and single-beam atmospheric spectrum with the line shape and instantaneous field-of-view of the instrument. On the other hand, Non-LTE effects, line mixing, and pressure shifts are not considered in the forward model, but are accounted for in the microwindows selection and in the error budget. The atmosphere is assumed horizontally homogeneous and in hydrostatic equilibrium.

Several mathematical and computing optimizations have been introduced in order to reduce the computing time with either null or negligible effect on the accuracy of the calculations (Raspollini et al., 2006).

The operational processor requires a pre-retrieval cloud detection scheme which filters the level 1b spectral data, as do all current MIPAS retrieval codes for trace gases. This is required because strong cloud effects in the spectra result in anomalous mixing ratios and instabilities of the retrieval solutions even for concentrations derived from lines-of-sight above the cloud-contaminated views. Both clouds in the troposphere and polar stratospheric clouds can produce these effects. The operational processor uses a cloud detection scheme based on the ratio of integrated signals in cloud microwindow pairs (Spang et al., 2004) with one pair defined in each of 3 MIPAS bands (A, B, and D). The tests operate hierarchically so that the cloud index in band A (CI-A) is tested first, and then the other bands if required because of an unavailability of band A. Hence CI-A is normally the cloud index used and the threshold for cloud detection is currently set such that CI-A $<1.8$ between $6 \mathrm{~km}$ and $45 \mathrm{~km}$ in the operational processor flags a spectrum as cloudy (further details can be found in Spang et al., 2004).

One of the main effects of cloud presence, particularly in the tropical upper troposphere, is to limit the cloud-free lineof-sight frequency. Greenhough et al. (2005) show that this obscuration of the MIPAS views can result in less than $20 \%$ cloud-free observations at $12 \mathrm{~km}$ in the inter-tropical convergence zone (ITCZ) which is observed to drift north and south according to season. However, outside the narrow band of latitudes of the ITCZ, the cloud-free line-of-sight frequencies at $12 \mathrm{~km}$ increase towards $40 \%$ in the tropics and greater than $60 \%$ in mid-latitudes. Retrieval of trace gas concentrations at upper tropospheric altitudes requires careful validation and joint retrieval with aerosol extinction (e.g. Moore, 2006) or continuum parameters (e.g. von Clarmann et al., 2003a). Work on many species is on-going but essentially considerable success is being achieved at these low altitudes (see Sect. 8). Aside from the cloud influences, water vapour, the cold temperatures at the tropical tropopause and line-of-sight gradients are most responsible for increased uncertainties. The last of these has led to research into two-dimensional retrieval codes as described below.

\subsection{Scientific codes for data processing}

Besides the operational MIPAS level-2 data processor operated under ESA responsibility, there exist several further data processors which are used to produce scientific level-2 data beyond the routine near real time data generation. Most of these processors involve modelling of aspects of radiative transfer not included in the ORM or use a retrieval strategy of higher sophistication. This usually implies a higher 
computational burden and/or expert knowledge of the user, which explains why not all of these codes are suitable for operational level-2 data generation.

Without being exhaustive, we shortly introduce the most widely used MIPAS level-2 data analysis codes. In the prelaunch phase of ENVISAT six codes were developed and cross-validated within the "Advances MIPAS Level-2 Data Analysis" (AMIL2DA) project (von Clarmann et al., 2003c).

These are the extended scientific versions of the OFM/ORM by IFAC, the Retrieval Control Program (RCP) by IMK/IAA, two distinct data processors by DLR, the RET2D code by the Rutherford Appleton Laboratory, and the Oxford University Processor to Invert MIPAS Measurements (OPTIMO), which meanwhile has been replaced by the MORSE software (Dudhia et al., 2005). Further MIPAS data processors are the one coded and applied by Forschungszentrum Jülich (Hoffmann et al., 2005), the OPERA code of the University of Leicester (Moore et al., 2006), and the GMTR code by Bologna University (Carlotti et al., 2006).

As for the operational ESA retrieval code, also the scientific data processors require the forward modelling of atmospheric radiative transfer. This can be done either by explicit line-by-line radiative transfer models (KOPRA (Stiller, 2000) in the case of RCP, RFM (Dudhia, 2002b) in the case of OPTIMO, MORSE, and OPERA), by the use of precalculated absorption cross-sections (ORM-based codes) or by computationally efficient but less accurate parametric radiative transfer codes as in the FZJ retrieval algorithm. The more advanced radiative transfer line-by-line models support modelling of radiative transfer physics which is, for reasons of computational efficiency, not included in the ORM, e.g. non-local thermodynamic equilibrium or scattering of radiation by clouds.

In the infrared region scattering is usually neglected in the radiative transfer, that means an aerosol free atmosphere is assumed and cloud filtering is applied (see above). Höpfner et al. $(2006 \mathrm{a}, \mathrm{b})$ have shown that intermediate cases of great scientific interest can also be considered. Since radiation from the lower atmosphere and the surface attenuated by the absorption of the atmosphere below the line of sight, is scattered e.g. by thin clouds (e.g. cirrus or PSCc) into the line of sight of MIPAS, the KOPRA radiative transfer algorithm has been complemented by a single scattering module (Höpfner et al., 2005).

Besides differences in radiative transfer modelling, there are also important differences between the processors with respect to the inversion strategy. While the ORM retrieves atmospheric temperatures and mixing ratios of constituents on an altitude grid defined by the tangent altitudes of the actual limb scan (Ridolfi et al., 2000), other algorithms use a fixed altitude grid, which in case of the RCP is much finer than the MIPAS tangent altitude spacing (1 km vs. $3 \mathrm{~km}$ ) (von Clarmann, 2003b). Most of the processors either rely on the engineering information on the MIPAS elevation pointing (e.g. RET2D) or correct pointing-related errors by ad- justing the pressure profile to the actual limb measurement. The RCP supports direct retrieval of the elevation pointing in terms of tangent altitudes while it adjusts pressure assuming the hydrostatical equilibrium, and is thus well suited for monitoring of elevation pointing peculiarities (von Clarmann et al., 2003b, Kiefer et al., 2007, this Special Issue).

While the majority of retrieval algorithms use microwindows for the retrieval, for species with excessively low signal like $\mathrm{HOCl}$, however, it happens that the use of extended spectral windows is necessary, whose width often is beyond of what is usually called microwindow (von Clarmann et al., 2006a). Other data analysis strategies rely on broadband data rather than microwindows (RET2D, FZJ-code, OPERA).

All retrieval codes except the ORM-derivates and GMTR constrain the retrieval of atmospheric state variables by application of a dedicated regularization term. This helps to avoid instabilities in the retrieval, but adds a certain content of a priori information to the retrieved profiles. OPTIMO, OPERA, and the FZJ-code use optimal estimation (Rodgers, 1976, 2000), MORSE uses a sequential estimation application of optimal estimation, the DLR-codes use different variants of a Tikhonov-type (Tikhonov 1963a, b) constraint, and RCP supports any user-defined regularization matrix but is usually operated with a Tikhonov-type first order finite differences regularization matrix (Steck et al., 2001).

Forward-modelling related issues of Non-LTE seem to be sufficiently well understood not to be the driving error source in calculated spectra, if the vibrational temperatures are accurately known. Since, however, the vibrational temperatures of the actual atmosphere are typically unknown, Non-LTE is not only a forward modelling issue but also poses retrievalrelated problems (von Clarmann et al., 2003b). At least, the vibrational temperatures should be modelled for the actual atmosphere. In case of $\mathrm{CO}$, whose vibrational temperatures depend on the CO infrared emissions at other altitudes, this requires the inclusion of a Non-LTE model which provides the vibrational temperatures within each step of iteration, as supported by the RCP-processing environment (Funke et al., 2006). This approach, however, relies on a perfect Non-LTE model. Since high-resolution spectra as provided by MIPAS in its original observation mode include a lot of information on Non-LTE processes themselves, uncertain Non-LTE model parameters like $\mathrm{NO}_{2}$ excitation rates, $\mathrm{O}_{3}$ nascent distributions, or $\mathrm{CO}_{2}$ collisional constants can also be retrieved directly from the measurements using the RCP processing, as shown by Funke et al. (2005a), Kaufmann et al. (2006), and López-Puertas et al. (2005c). With no Non-LTE model available, the only possible approach except the use of climatological vibrational temperature data (López-Puertas et al., 2006) is the direct retrieval of vibrational temperatures from the spectra. This, however, involves an inversion with many unknowns which tends to be ill-posed and thus needs regularization. Any physically meaningful regularization of vibrational temperatures, however, can be set up best on the basis of a Non-LTE model. 
With these scientific retrieval processors available, it is possible (a) to extend the MIPAS data product towards lower altitudes, where clouds affect radiative transfer and where reduced measurement information in nearly saturated spectra requires regularization of the retrieval; (b) towards higher altitudes where the correct treatment of Non-LTE is important; (c) to investigate regions of the atmosphere where large horizontal gradients make the horizontal homogeneity assumption fail, and (d) to derive many more trace species characterized by excessively low signal/noise ratio which requires more sophisticated retrieval strategies than used for the operational data products.

\subsection{Two-dimensional and multi-target retrievals}

In recent years, significant efforts have also been made for the development of two-dimensional (2-D) retrieval codes that avoid the assumption of horizontal homogeneity of the atmosphere. Indeed, conventional retrieval schemes apply a one-dimensional approximation of the atmosphere, i.e., for each limb scan the retrieved physical state variable is a function of altitude only, while horizontal variations are neglected (see, e.g. McKee, 1969, Carlotti, 1988). In order to allow the retrieval of two-dimensional fields of atmospheric state variables in the orbit plane, dedicated algorithms have been developed.

MIPAS observations taken along the orbit track can be analyzed in two dimensions by exploiting the fact that the limb sequences are continuously repeated along the plane of the orbit. This repetition allows to gather the information about a given location of the atmosphere from all the lines of sight that cross that location whatever sequence they belong to. Since the loop of overlap between nearby sequences closes when the starting sequence is reached again at the end of the orbit, in a retrieval analysis the full gathering of information can be obtained by merging in a simultaneous fit the observations of a complete orbit; this analysis strategy is named Geo-fit (Carlotti, 2001). An important feature of the Geo-fit approach is that the retrieval grid is independent of the measurement grid so that atmospheric profiles can be retrieved with horizontal separations different from those of the measured limb scans. This feature often makes it possible to improve the usually poor horizontal resolution of limb measurements (Ridolfi et al., 2004).

MORSE, RET2D and the 2D-option of the RCP (Steck et al., 2005) use sequential estimation, where the a priori information on the 2-D field is updated with each limb scan newly included in the analysis. As a computationally more efficient substitute for full 2-D retrievals, the RCP also supports the retrieval of horizontal gradients along with the related atmospheric state variables.

In the ESA operational data-analysis process, the preliminary retrieval of pressure and temperature is followed by the sequential retrieval of the target vmrs. A drawback of this approach is that the retrieval errors affecting pressure and temperature profiles do propagate into the retrieved vmr values. Moreover, molecular species with a "rich" spectrum (such as water and ozone) may also propagate their measurement error in the other products because their spectral features often contaminate the wavenumber intervals analyzed for the retrieval of other species. This error propagation process is minimized with a careful choice of both the analyzed spectral intervals (Dudhia et al., 2002a) and the sequence of the retrievals. Nevertheless, in many cases, the error propagation cannot be completely avoided and its assessment is rather difficult. A strategy that eliminates this source of systematic errors is represented by the simultaneous retrieval of all the quantities whose correlation in the observed spectra is the cause of the error propagation. With this strategy (referred to as Multi-Target Retrieval; MTR) the error due to the cross-talk between different target quantities is properly represented in the covariance matrix of the retrieved parameters (Dinelli et al., 2003). Furthermore, the selection of the observations to be analyzed is no longer driven by the necessity to reduce the interferences among the target species. Finally, the information on pressure and temperature can be gathered from the spectral features of all the target species that are simultaneously retrieved and not only from $\mathrm{CO}_{2}$ lines.

Both the Geo-fit and MTR functionalities are implemented in the GMTR (Geofit Multi Target Retrieval) opensource code available at http://www.science-and-technology. nl/beat/ and http://www.mbf.fci.unibo.it.

\subsection{Spectroscopic database for MIPAS retrievals}

Although general databases such as HITRAN (Rothman et al., 2005) or GEISA (Jacquinet-Husson et al., 2005) existed it was decided at the beginning of the development of the MIPAS data processing software to generate a dedicated spectroscopic database for MIPAS, as was done for the ATMOS experiment (Gunson et al., 1996), in order to be able to implement the best spectral parameters in the shortest possible time and also to meet the specific needs of MIPAS. The first version "mipas_pf2.0" was based on both the 1996 version of HITRAN and on new laboratory spectroscopic data and new calculations (Flaud et al., 2003a; Flaud et al., 2003b). After launch, version "mipas_pf3.1" (Flaud et al., 2003c) of the MIPAS dedicated spectroscopic database was released. With respect to the former one it contained improvements concerning mainly the following molecules: $\mathrm{CO}_{2}, \mathrm{HNO}_{3}$, $\mathrm{CH}_{4}, \mathrm{NO}_{2}, \mathrm{O}_{3}$, and $\mathrm{COF}_{2}$. In particular, the new spectroscopic data for $\mathrm{HNO}_{3}$ in the $11.2 \mu \mathrm{m}$ spectral region are responsible for a change of about $14 \%$ in the retrieved $\mathrm{HNO}_{3}$ volume mixing ratios (Flaud et al., 2003b). This change was assessed and validated by simultaneous retrievals of atmospheric $\mathrm{HNO}_{3}$ profiles in the $11.2 \mu \mathrm{m}$ spectral region and in the pure rotation region (Mencaraglia et al., 2006). More recently a new version "mipas_pf3.2" including changes concerning the $\mathrm{NO}, \mathrm{OCS}, \mathrm{NH}_{3}, \mathrm{H}_{2} \mathrm{O}_{2}$, and $\mathrm{HNO}_{3}$ molecules has been made available and the MIPAS spectra have been used 
to validate the $\mathrm{HNO}_{3}$ line parameters on the whole MIPAS spectral domain (Flaud et al., 2006). Finally it proved possible using new spectroscopic data to observe the $\mathrm{H}^{15} \mathrm{NO}_{3}$ species in MIPAS spectra (Flaud et al., 2007).

\section{Observation strategy and measurement modes}

\subsection{Rationale}

\subsubsection{Introductory remarks}

The scientific objectives as described in Sect. 2 and the observing capabilities as outlined in Sect. 3 are manifold requiring a flexible observation strategy. The observing capabilities allow vertical sampling steps of 1 to $8 \mathrm{~km}$, tailored to the scientific objectives. The altitude coverage that can be commanded is 5 to $160 \mathrm{~km}$, thus including the range from the free troposphere up to the thermosphere. Rearward and sideward views are possible with an azimuth scanning range of $35^{\circ}$ and $30^{\circ}$, respectively. In the rearward (i.e. along-track) view this allows e.g. (1) a complete pole to pole coverage by compensating for the inclination of the orbit or (2) to measure along tracks parallel to the satellite subtrack. In the sidewards (i.e. cross-track) view the azimuth range permits the observation of special targets, e.g. to view parallel to the terminator or to stare at a special event (e.g. volcanic eruption). Consequently, a high degree of freedom concerning possible observation modes is realised. On the other hand operational constraints, the reliability of retrievals, and the standardization of processing routines had to be taken into account. Based on this, a set of different observation modes was specified and a mission plan, which defines the distribution of observation modes over the lifetime of ENVISAT, was established.

The following sections describe the observation modes that were effective for the first two years of MIPAS in space. Upon increasing failure rates in the interferometer drive unit in 2004 a major change in the operation of the interferometer was introduced with reduced optical path difference. Constrained by this the spectral resolution was reduced to about $40 \%$ of the prior value and the duty cycle of MIPAS was restricted in 2005 to $35 \%$. Recently, the duty cycle has been increased step by step reaching $100 \%$ in December 2007. As a consequence, the observation modes and the mission plan had to undergo major revisions which are not subject of this paper since this paper focuses onto the first two years when MIPAS operated with full spectral resolution.

\subsubsection{Spatial coverage}

The scientific objectives make it clear that polar, as well as mid- and low-latitude coverage is required. Furthermore, given the importance of flexibility, it was requested to ensure that MIPAS could observe any region of the globe as otherwise unexpected phenomena could be missed. Consequently, it was requested to make both the elevation and azimuth angles of MIPAS adjustable.

The elevation scan was requested to cover altitudes extending from the middle troposphere to the lower thermosphere. Measurements in the upper troposphere are needed e.g. to study troposphere-stratosphere exchange processes and pollution by aircraft emissions. However, the primary scientific objective of MIPAS is the investigation of stratospheric processes, in particular chemical reactions and transport, which necessitates the simultaneous measurement of the distribution of many trace constituents in this part of the atmosphere. Measurements in the meso- and thermosphere are mainly used for studying Non-LTE processes and interactions between the thermo-, meso- and stratosphere.

\subsubsection{Spatial resolution}

More recent field measurements have shown that the variability in the spatial distribution of atmospheric constituents in the upper troposphere and lower stratosphere can be quite large, in particular across the polar vortices and close to the tropopause. However, classical limb viewing remote sensing sensors can provide only limited spatial resolution. MIPAS has an IFOV of about $3 \mathrm{~km} \times 30 \mathrm{~km}$ which, as a rule of thumb, limits the resolution in the vertical domain to $3 \mathrm{~km}$, and in the horizontal domain to about $300 \mathrm{~km}$ along the line of sight and $30 \mathrm{~km}$ perpendicular to the line of sight. As a consequence, it is not possible to resolve small scale phenomena in the atmosphere with such instruments.

On the other hand, space-borne remote sensing can provide good global surveys of stratospheric composition. For studying the chemistry of the stratosphere a horizontal resolution of about $500 \mathrm{~km}$ is mostly sufficient and equivalent to the resolution of state-of-the-art chemical transport models.

Concerning troposphere-stratosphere exchange processes it is obvious that single medium and small scale processes like the effect of a single convective tower or tropopause folds cannot be resolved with MIPAS. On the other hand, for the main upward transport from the troposphere into the stratosphere the horizontal resolution of MIPAS should suffice.

The relatively high signal-to-noise ratios of spectra observed in the UTLS (Upper Troposphere Lower Stratosphere) allows to gain from oversampling, i.e. using sampling grids smaller than the width of the IFOV and thus to achieve vertical resolutions of better than $3 \mathrm{~km}$ for certain parameters.

\subsubsection{Temporal coverage and resolution}

Various time scales are of importance, namely those associated with diurnal and with long term variations. The detection of diurnal variations can provide a powerful test of photochemical theory but, in principle, a sun-synchronous orbit 
only allows two local times to be sampled at each latitude every day. When the spacecraft is crossing the terminator a considerable number of measurements at different times of the day are possible in case of the flexible scan mechanism of MIPAS.

The detection of longer term trends will be difficult due to the problem of instrument drift and degradation. However, these requirements are taken into account in the calibration concept of MIPAS in an attempt to ensure that the retrieved data will be accurate enough to contribute also to trend studies for various species (e.g. for HCFC-22).

\subsection{Observation modes}

According to the scientific objectives several observation modes have been identified, each associated with different objectives, altitude coverage, vertical and horizontal sampling, and, for some cases, spectral resolution. Typical observation modes are summarized in Table 1. The nominal mode was defined as standard, addressing most of the scientific objectives and covering about $80 \%$ of the measurement time. All other modes are focused on special research tasks such as for example polar winter chemistry, the study of the upper atmosphere, or troposphere-stratosphere exchange.

\subsubsection{Nominal observation mode}

This mode has been the standard mode for stratospheric chemistry and dynamics though it has been extended down into the upper troposphere and up into the lower mesosphere to allow to study linkages between the stratosphere and its adjacent atmospheric compartments, the troposphere and mesosphere. The vertical coverage of this mode is from 6 to $68 \mathrm{~km}$ globally. The vertical spacing in this mode was generally adapted to the IFOV of the instrument, i.e. to $3 \mathrm{~km}$. In the upper stratosphere and lower mesosphere, however, the spacing is gradually relaxed from $3 \mathrm{~km}$ to $8 \mathrm{~km}$ in order to maintain a horizontal (along-track) sampling of $\sim 500 \mathrm{~km}$. Observations are performed in the rearward pointing range, with the azimuth angle set as a function of orbit position to permit full pole-to-pole coverage.

\subsubsection{Polar winter chemistry mode}

In polar regions, during winter and spring, the lower stratosphere is of major interest because of PSC formation, perturbed chemistry, and potential ozone "hole" formation. The high spatial variability of the relevant processes and associated fields of geophysical parameters requires an optimum vertical and good horizontal resolution of the measured data. This was achieved with a denser altitude and along-track sampling in the UTLS at the expense of a reduced coverage of the altitude region in the upper part of the atmosphere. For this purpose the lowest point of the altitude scan was set to be about $4 \mathrm{~km}$ below the climatological tropopause in order to ensure that the lowermost limb view originated in the actual troposphere, i.e. to account for (1) the finite width of the instantaneous field-of-view (IFOV), (2) uncertainties of the acquisition, and (3) for actual fluctuations of the tropopause height compared to the climatological value. A latitude dependent altitude offset was introduced according to

Minimum tangent altitude $=$

$8 \mathrm{~km}+2 \mathrm{~km} \cdot \cos (2 \cdot$ tangent point latitude $)$

in order to allow the lowermost tangent altitude to follow the climatological variation of the tropopause.

\subsubsection{Upper Atmosphere modes}

As a limb emission sounder with broadband spectral coverage along with high spectral resolution MIPAS can provide unprecedented information on the upper atmosphere (UA). These observations improve our understanding of the middle and upper atmosphere, e.g. with respect to the temperature structure and energy balance, the chemistry, the dynamics, and the interaction between them. In addition, the nonlocal thermodynamic equilibrium (Non-LTE) of the middle and upper atmosphere can be studied in better detail than ever before (López-Puertas et al., 2005c). For this purpose the observing capabilities have been extended up to $160 \mathrm{~km}$. Several reference tables with different altitude ranges and incremental steps have been prepared for various purposes of upper atmospheric research. For obtaining a complete scan from the stratosphere up into the lower thermosphere a reduced horizontal and/or vertical resolution has to be accepted.

At the beginning of the mission the most important objective of the UA measurements was to verify/falsify the predicted Non-LTE effects on the on-line/off-line retrievals. Other UA modes with various choices of altitude sampling and coverage were devoted to (1) Upper polar vortex dynamics and stratosphere-mesosphere exchange processes, (2) Energy budget, Non-LTE studies and budgets of hydrogen, nitrogen, and carbon in the upper atmosphere, (3) Non-LTE studies related to NO specifically extending the measurements up to $160 \mathrm{~km}$.

\subsubsection{Troposphere-stratosphere exchange mode}

For the investigation of troposphere-stratosphere exchange a rather dense spacing of observations in the vertical and horizontal domain is needed. Fortunately, in this case the required altitude range can be reduced. The achievable horizontal resolution depends on the number of sampling points in the vertical domain. The vertical resolution is restricted by the IFOV of $3 \mathrm{~km}$ and the limb geometry. Nevertheless, oversampling in the altitude domain allows to improve the vertical resolution. A minimum step size of $1.5 \mathrm{~km}$ has 
Table 1. MIPAS Observational Modes.

\begin{tabular}{|c|c|c|c|c|c|}
\hline \multirow{4}{*}{ Scientific Objective } & \multicolumn{5}{|c|}{ Proposed Measurements } \\
\hline & Global & Regional & Altitude & \multirow{3}{*}{$\begin{array}{l}\text { Spacing } \\
\text { Vert. } \\
(\mathrm{km})\end{array}$} & \multirow{3}{*}{$\begin{array}{l}\text { Horiz. } \\
(\mathrm{km})\end{array}$} \\
\hline & & or & Range & & \\
\hline & & \multicolumn{2}{|l|}{ Occasional } & & \\
\hline Stratospheric Chemistry & $\mathrm{X}$ & & $6-68^{(1)}$ & $3-8$ & $510^{(2)}$ \\
\hline Polar Winter Chemistry & $(\mathrm{X})$ & $\mathrm{X}$ & $7-48^{(1)}$ & $2-8$ & $420^{(2)}$ \\
\hline Middle and Upper & & $\mathrm{X}$ & $20-160^{(1)}$ & $3-8$ & $\sim 800^{(2)}$ \\
\hline Atmosphere & & & & & $(\sim 450)^{(4)}$ \\
\hline \multicolumn{6}{|l|}{ Exchange between } \\
\hline \multicolumn{6}{|c|}{ Stratosphere and Troposphere, } \\
\hline Tropospheric Chemistry & $(\mathrm{X})$ & $\mathrm{X}$ & $5-38^{(1)}$ & $1,5^{(5)}-8$ & $\sim 350^{(2)}$ \\
\hline Dynamics & & $\mathrm{X}$ & $7-71^{(1)}$ & $3-8$ & $120-200^{(4)}$ \\
\hline Diurnal Changes & & $\mathrm{X}$ & $15-60$ & 3 & $\sim 100^{(3)}$ \\
\hline Special Events & & $X$ & tbd & tbd & tbd \\
\hline
\end{tabular}

Notes

1 Vertical spacing variable.

2 Along track (rear view).

${ }^{3}$ Cross track (side view).

4 Spectral resolution reduced.

5 Because of overlapping weighting functions and the limited IFOV the altitude resolution of the retrieved profiles does not increase proportionally to the spacing. In case of the Polar Winter Chemistry Mode a latitude-dependant altitude range is used.

been regarded as sufficient. In this mode the identification of clouds in the field-of-view is very important.

\subsubsection{Dynamics mode}

To study medium to small scale structures in the middle atmosphere like gravity waves, ozone laminae, and stratospheric intrusions a specific dynamics mode was foreseen. In this case the horizontal resolution/coverage is considerably increased and at the same time the altitude range and the vertical resolution of the nominal scan mode has to be kept constant. This can be achieved by reducing the spectral resolution (i.e. the maximum optical path difference of the interferometer sweep) by a factor between 4 and 10. Although the reduced spectral resolution reduces the number of retrievable species, the rather long-lived species $\mathrm{O}_{3}, \mathrm{H}_{2} \mathrm{O}$, and some important tracers like $\mathrm{N}_{2} \mathrm{O}, \mathrm{CH}_{4}$, and some CFCs are still measurable (see Fischer et al., 2000).

\subsubsection{Cross track and special events modes}

Diurnal changes

The study of diurnal changes near the terminator requires a high latitudinal resolution. This can be achieved with MIPAS by making cross track observations and adjusting the azimuth angle during the elevation scan in order to maintain the observation of tangent heights at nearly the same latitude/longitude spot for a complete limb scan sequence. 


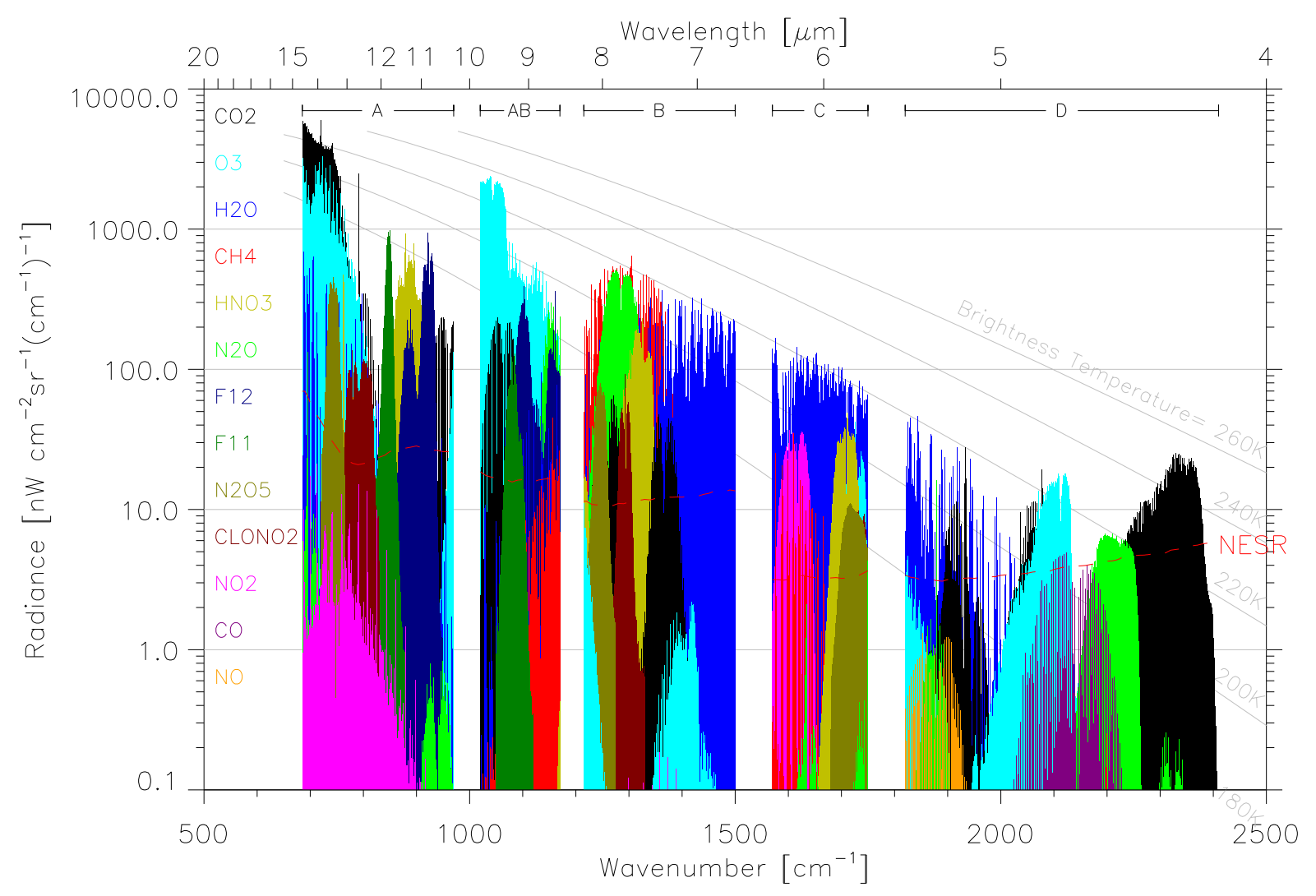

Fig. 7. The region of the infrared spectrum covered by the 5 bands of MIPAS, showing the coloured emission features of some of the most significant molecules, calculated for a limb view of tangent altitude $12 \mathrm{~km}$ (approx. $200 \mathrm{hPa}$ ). The red dashed line represents a typical value of the Noise Equivalent Signal Radiance (NESR).

Impact of aircraft emission (AE)

The AE mode is dedicated to the detection of aircraft emissions and their effects on the chemistry in the upper troposphere and lowermost stratosphere. The sampling is optimized to the 7 to $13 \mathrm{~km}$ altitude range (1.5 km sampling) where the expected effect is largest. Some layers are added on top in order to restrict the sensitivity of the retrieval to altitude regions above the aircraft corridor. The number of samples per sequence is 12 . The azimuth angles are optimized such that the lines of sight are as parallel as possible to the aircraft corridors to enable long optical paths inside the aircraft corridors and a high contrast between measurements within the aircraft corridors and those in adjacent 'unaffected' regions of the atmosphere.

\section{Special events mode}

A stare scan mode can be commanded for special events such as volcanic eruptions though the required vertical and horizontal spacing of the measurements will depend strongly on the spatial extent of the event under investigation.

\section{Targeted constituents and estimates of measurement errors}

\subsection{The spectral coverage of MIPAS}

From a scientific point of view it would be desirable to cover the whole atmospheric emission spectrum simultaneously. However, technical constraints restrict the spectral coverage in case of MIPAS to the mid-infrared from 4.15 to $14.6 \mu \mathrm{m}$.

Figure 7 shows the region of infrared spectrum observed by MIPAS. Due to the scale of this plot $\left(1 \mathrm{~cm}^{-1}\right)$ the spectral features of the different molecules cannot be separated, but with the spectral resolution actually obtained by MIPAS (Fig. 8) individual lines can be resolved. Although MIPAS can, in principle, retrieve many different species, the requirement for a robust retrieval led to the fairly conservative choice of the seven molecules with strongest line features $\left(\mathrm{CO}_{2}\right.$ for the pressure/temperature $(\mathrm{pT})$ retrieval, plus six key species) in case of the operational data processing (see also Sect. 4). 


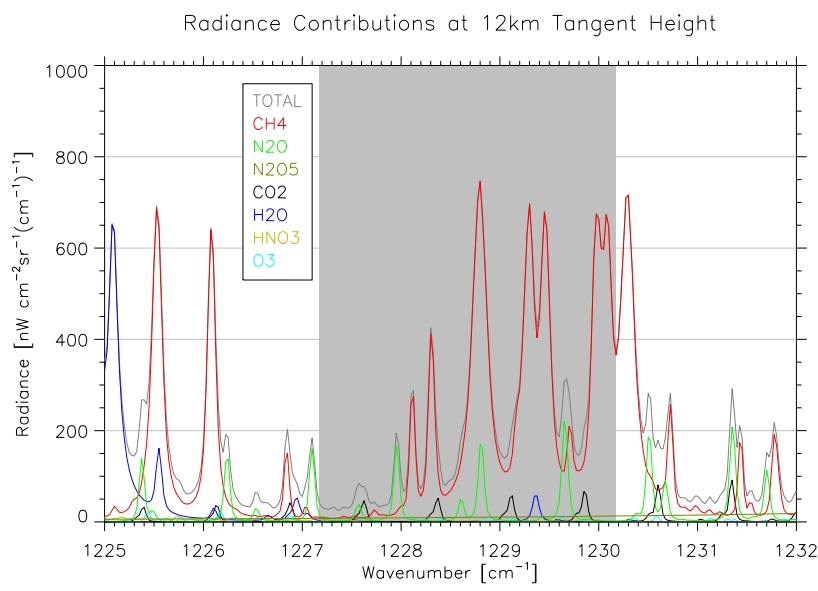

Fig. 8. Expansion of Fig. 7, showing the $1225-1232 \mathrm{~cm}^{-1}$ region in detail at $0.025 \mathrm{~cm}^{-1}$ resolution. The grey curve, mostly obscured by the $\mathrm{CH}_{4}$ contribution, shows the total radiance spectrum. The shaded region shows the spectral position of the primary $\mathrm{CH}_{4}$ microwindow used in the operational retrieval.

\subsection{Microwindows}

Computational costs restrict the number of spectral points that can be used in the operational retrieval to only a few percent of the complete spectrum. Rather than using individual spectral points, the adopted approach is to use "microwindows", i.e. spectral regions of up to $3 \mathrm{~cm}^{-1}$ width (von Clarmann and Echle, 1998). One of the main advantages of using microwindows instead of single lines is that a flat atmospheric continuum term can also be fitted, and therefore its effect can be greatly reduced within each microwindow, making the retrieval less sensitive to atmospheric aerosols and thin clouds.

Between two and seven microwindows are used for each target species, as shown in Fig. 9. For pT, $\mathrm{O}_{3}$, and $\mathrm{H}_{2} \mathrm{O}$, the tangent altitude range of the microwindows varies according to the strength of the spectral absorption feature, i.e. using the strongest absorption regions for high altitudes and weaker features for low altitudes. These microwindows are selected (Dudhia et al., 2002a) to give the best compromise between maximizing the precision of the target species retrieval and minimising the contribution of interfering species and other systematic error sources (as listed in Table 2).

\subsection{Error analysis}

The microwindow selection aims to maximize the accuracy of the retrieval by modelling the propagation of not only the instrument noise but also other errors in both the measurements and the forward model. Therefore it contains all the information required for a full error analysis of the retrieval. Figure 10 shows an example of the error analysis for $\mathrm{CH}_{4}$ for the operational product. Between the upper troposphere and the stratopause the total error (TOT) is nearly constant. The single errors as shown in the figure are more or less dependent on altitude.

The term systematic error (SYS) is used here to describe all errors apart from the noise (RND). Each of these error sources is assumed to introduce some component of correlated error into the measurement (or forward model calculation).

The systematic error correlations are only defined for the set of measurements that contribute to a single profile, and some care needs to be taken if these error analyses are applied to larger datasets. For example, the spectroscopic database errors (SPECDB) would be common to all profiles generated throughout the mission (effectively appearing as a bias term) whereas the $\mathrm{pT}$ propagation errors might be random from one profile to the next and disappear with averaging. Other errors would have intermediate time-scales depending on atmospheric variability or calibration frequency.

Similar error analyses have been carried out for the other operational data products and also for the scientific results.

\section{Validation of the MIPAS data products}

\subsection{General comments}

As shown above MIPAS is an IR remote sensing experiment which yields absolutely calibrated radiances; they are converted into vertical profiles of atmospheric parameters with well defined error bars. The data processing software has been evaluated by intercomparisons of data processors of different institutions at an international level (von Clarmann et al., 2003c) and by applying it to measurements as performed by similar instrumentation (e.g. MIPAS balloon experiment). Nevertheless, the responsible scientist of satellite experiments always wants to validate the satellite data with results of independent instrumentation. Reasonably, the quality of these validation measurements should be better than that of the satellite data. However, this requirement is not always met.

In order to intercompare measurements of instruments on different platforms it is common practice to apply coincidence criteria. Differences in space and time are restricted dependent on the atmospheric situation (in particular for the validation of satellite measurements by single non-satellite observations). The limitations depend on horizontal gradients or diurnal variations of the observed parameters. Standard coincidence criteria are a distance of $300 \mathrm{~km}$ and a few hours time difference. These values are based on the experience on measurements of gradients of atmospheric parameters in the stratosphere. Larger differences are acceptable if the atmosphere is proven to be homogeneous or if the results at one location are transformed to the other measurement location with the use of 3-D numerical models or trajectory calculations. A general discussion on how to perform 

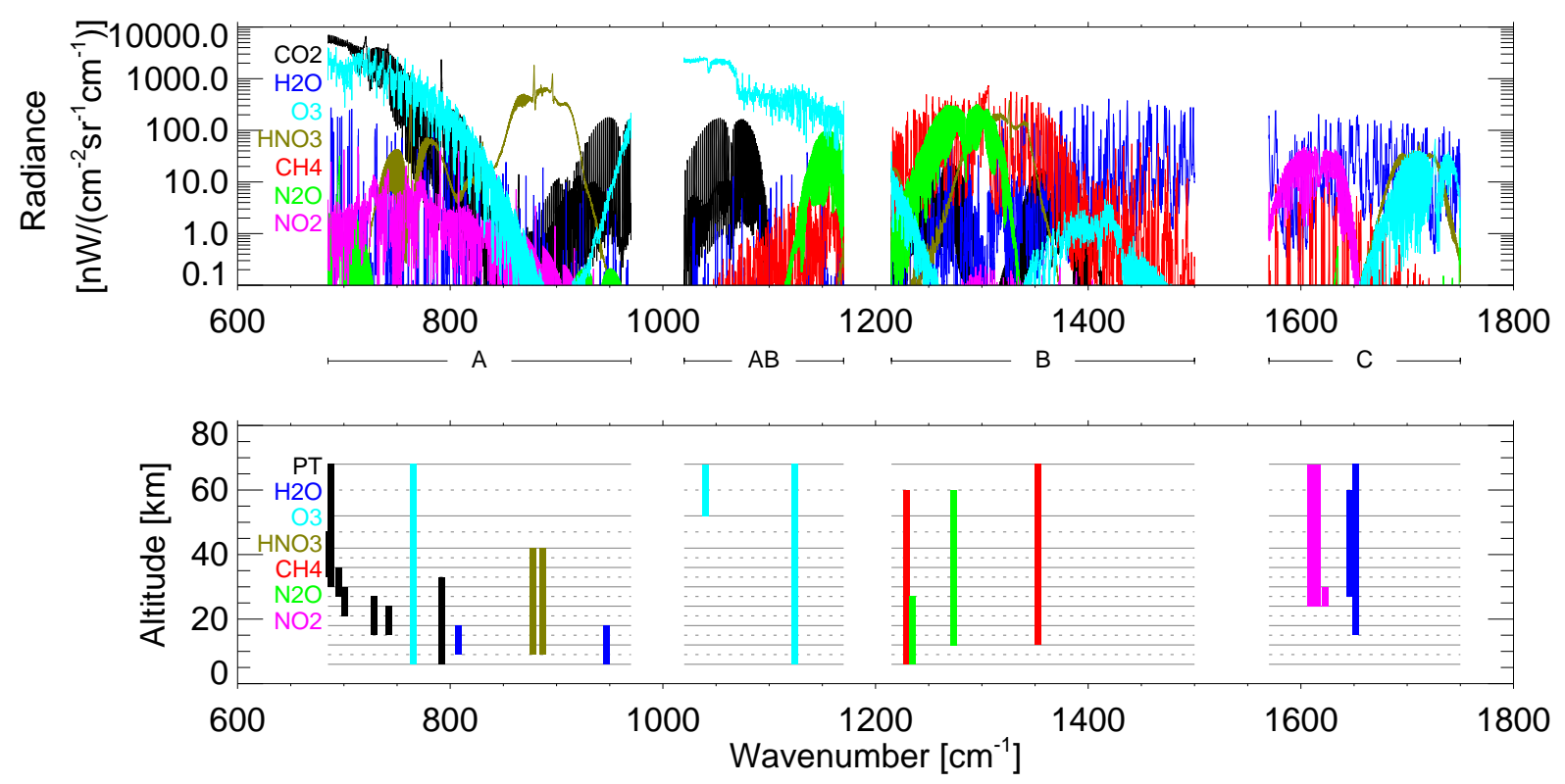

Fig. 9. The upper plot shows the emission features of the target molecules in the MIPAS spectrum calculated for $21 \mathrm{~km}$ tangent altitude. No operational microwindows are selected in band D (mainly due to poor signal/noise). The lower plot shows the spectral locations and tangent altitude range of the microwindows use for the MIPAS "off-line" retrievals (note that the actual spectral width of each microwindow - maximum $3 \mathrm{~cm}^{-1}$ - is too narrow to be shown to scale on these plots.

Table 2. MIPAS error sources, in addition to random noise, and estimates of $1 \sigma$ uncertainty, the terms under "Code" are used in the error analysis for single gases (see e.g. Fig. 10).

\begin{tabular}{|c|c|c|}
\hline Error Source & $1 \sigma$ & Code \\
\hline \multicolumn{3}{|c|}{ Errors in Instrument Characterisation } \\
\hline Radiometric Gain & $\pm 2 \%$ & GAIN \\
\hline Spectral Calibration & $\pm 0.001 \mathrm{~cm}^{-1}$ & SHIFT \\
\hline Apodised ILS Width & $\pm 2 \%$ & SPREAD \\
\hline \multicolumn{3}{|c|}{ Errors in Forward Model Parameters } \\
\hline Profiles of 28 gases & Climat. SD ${ }^{1}$ & [gas] \\
\hline High Alt. Column & Climat. SD ${ }^{1}$ & HIALT \\
\hline Line database errors & $\left(\right.$ See Note $\left.^{2}\right)$ & SPECDB \\
\hline Continuum model & $\pm 25 \%$ & CTMERR \\
\hline Retrieved pT error & pT noise covariance & PT \\
\hline \multicolumn{3}{|c|}{ Deficiencies in Forward Model } \\
\hline Non-LTE effect & Modelled & NONLTE \\
\hline $\mathrm{CO}_{2}$ Line Mixing & Modelled & CO2MIX \\
\hline Horiz. T gradients & $\pm 1 \mathrm{~K} / 100 \mathrm{~km}$ & GRA \\
\hline
\end{tabular}

Notes

${ }^{1}$ Uncertainty with which climatology represents the actual local state of the atmosphere.

${ }^{2}$ Based on assumed accuracies in line parameters.

the validation of measurements is contained in von Clarmann (2006b).

In the MIPAS Special Issue of ACP mainly the operational data products of the MIPAS experiment, namely temperature and mixing ratios of $\mathrm{O}_{3}, \mathrm{H}_{2} \mathrm{O}, \mathrm{CH}_{4}, \mathrm{~N}_{2} \mathrm{O}, \mathrm{HNO}_{3}$, and
$\mathrm{NO}_{2}$ are validated. By doing this it is proven that the MIPAS instrument is behaving well and the predicted error budgets are assessed at least in certain altitude regions. As a consequence, the data products are of good quality and can be applied for operational and scientific use. In the meantime, 


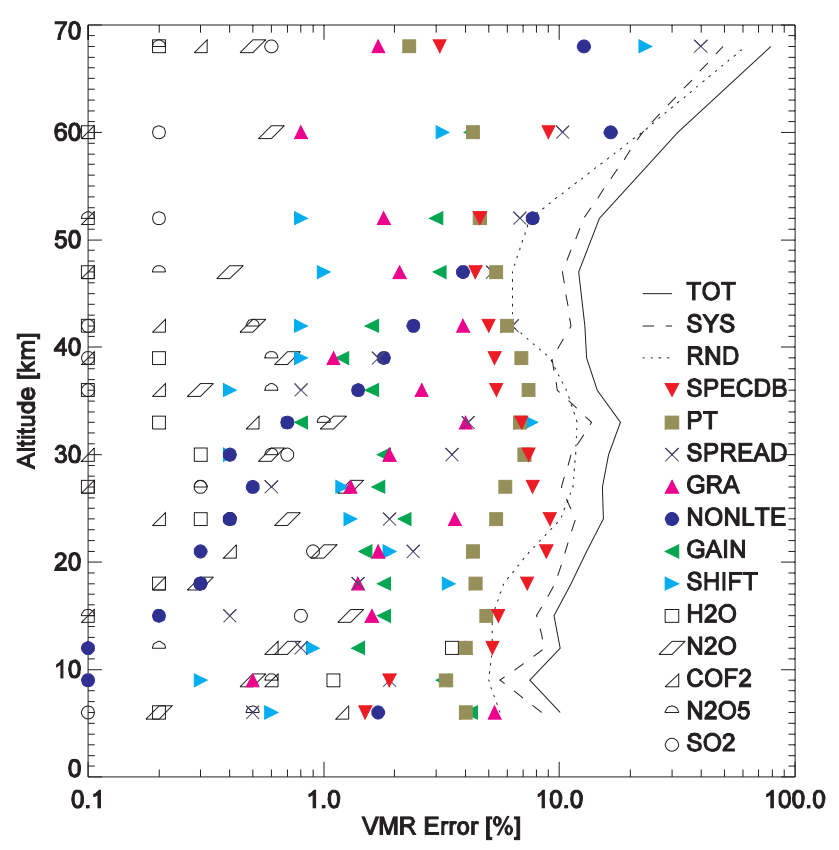

Fig. 10. Error analysis for the Level $2 \mathrm{CH}_{4}$ product for mid-latitude day-time conditions. The solid line represents the total error, divided into random (dotted) and systematic (dashed) contributions. The various symbols represent the different components of the systematic error (see Table 2), in approximate order of importance.

also a considerable number of papers on the validation of MIPAS data products as derived from scientific data processors have been published and have demonstrated the capabilities of MIPAS.

\subsection{Types of validation measurements}

Among the approaches to be considered for the validation of the MIPAS data products are comparisons with data obtained by

- instruments operated during dedicated balloon and aircraft campaigns,

- ground-based networks and

- other satellite instruments.

Remote sensing instruments flown on large stratospheric balloons can provide good vertical resolution concentration profiles below their flight altitude (up to $40 \mathrm{~km}$ ) and column abundances above it for a large number of atmospheric species. Other measurement techniques are based on insitu cryogenic sampling and on chemiluminescence devices; these are being used during the ascent and descent segments of stratospheric flights. All the above mentioned approaches can provide measurements with the vertical resolution needed to validate space-based observations of the upper troposphere and the stratosphere.
In comparison to balloon measurements, aircraft experiments are generally restricted in the altitude range of the measured vertical profiles. On the other hand, aircraft experiments are capable of covering quite large geographic regions, allowing for example the investigation of latitudinal changes and in-and-out-of-vortex measurements in polar regions.

Ground-based facilities can provide regular observations of many trace species throughout the entire lifetime of a space mission. They can thus help to characterize the longterm instrument stability in orbit. Some of the groundbased instruments provide total vertical column abundances with limited information on vertical distribution while others (e.g. Lidars) yield detailed volume mixing ratio profiles of some trace gases.

Validation of MIPAS data with other satellite measurements has the great advantage of intercomparing many measurements and being able to derive results which are based on statistics. However, the accuracy of the satellite data employed is sometimes not better than that of the satellite experiment to be validated.

Obviously, the different types of validation experiments have all their specific advantages and disadvantages. In order to conclude about the quality of the MIPAS data we need all of them in the validation process.

\subsection{Validation campaigns}

In time ESA has planned a comprehensive validation programme for all the ENVISAT instruments. The central part of this programme was the ENVISAT Stratospheric Aircraft and Balloon Campaign (ESABC). It included within the first two years after the ENVISAT launch balloon campaigns in Kiruna/Sweden, Aire sur l'Adour/France, Sicily/Italy and Vanscoy/Canada, as well as a campaign with small balloons in Bauru/Brazil. During those campaigns a large number of vertical profiles of atmospheric trace constituents has been recorded which played an important role during the validation process (see validation papers in this Special Issue). The balloon data were complemented by measurements from the research aircrafts Geophysica (in Forli/Italy and Kiruna/Sweden) and Falcon (flights from the tropics to the Arctic). All the experiments have tried to optimize the coincidence with ENVISAT overflights and they succeeded very often. This validation programme has been prolonged until the end of 2007 with reduced intensity.

\subsection{Results of the validation}

It is not intended to describe the detailed results of the validation exercise in this paper. The interested reader is referred to the corresponding validation papers in this Special Issue. But a summary of the validation results using ESA operational data 4.61/4.62 will be given in the following.

The ESA workshops on validation in May 2004 and December 2006 as well as the validation papers in this Special 
Issue have proven that the MIPAS operational data products are throughout of good quality. The MIPAS data are also self-consistent as shown by the data assimilation project ASSET (Lahoz et al., 2007).

The MIPAS global temperature data show a small bias (generally smaller than 1 or $2 \mathrm{~K}$ depending on altitude) with respect to the validation data but this bias is consistent with the error estimates. Also the discrepancies between MIPAS temperature and the temperature fields resulting from the ECMWF analyses have been characterized. The bias and the standard deviation of these discrepancies are consistent with those obtained when comparing MIPAS data to correlative measurements; however, the detected bias to ECMWF data has a peculiar altitude dependence. But this seems to be a problem of the ECMWF temperature distribution (see Ridolfi et al., 2007).

From the beginning the MIPAS ozone data showed very good agreement with various validation data sets. A clear indication of the validity of MIPAS $\mathrm{O}_{3}$ vertical profiles is obtained for most of the stratosphere, where the mean relative difference with the individual correlative data sets is always lower than $\pm 10 \%$. Larger discrepancies in ozone concentrations have been recognized in the lower stratosphere but this effect is largely due to the large atmospheric variability and the imperfect cloud flagging in this height region (Cortesi et al., this Special Issue).

The MIPAS water vapour profiles show no bias in the altitude region between 10 and $100 \mathrm{hPa}$ with respect to various validation data. There seems to be a positive bias in the order of $10 \%$ above $10 \mathrm{hPa}$ and a low bias in the tropopause region. The operational MIPAS data show still some oscillations in the vertical profiles (see Oelhaf et al., to be submitted in May 2008).

When intercomparing MIPAS $\mathrm{N}_{2} \mathrm{O}$ profiles with groundbased FTIR data no bias can be identified. In case of $\mathrm{CH}_{4}$ the results are different: FTIR partial columns of the lower stratosphere show a negative bias in the order of $10 \%$ (see Payan et al., 2007, this Special Issue).

MIPAS $\mathrm{HNO}_{3}$ data generally agree with the various correlative data sets within the combined error bars. In many cases differences between the correlative measurement pairs are less than $10 \%$ in the entire altitude range up to $38 \mathrm{~km}$. The standard deviations for the satellites comparisons (ODIN, ILAS, ACE) are generally larger than the estimated measurement uncertainty, but this is associated with the temporal and spatial coincidence error and the horizontal smoothing error which are not taken into account in the error budget (Wang et al., 2007). MIPAS $\mathrm{NO}_{2}$ profiles yield valuable information on the vertical distribution of $\mathrm{NO}_{2}$ in the lower and middle stratosphere during day and night with an overall accuracy of 10 to $20 \%$ and a precision of typically 5 to $15 \%$. When extremely high $\mathrm{NO}_{2}$ occurs in the mesosphere in polar winter the operationally generated $\mathrm{NO}_{2}$ profiles are less accurate; in these cases $\mathrm{NO}_{2}$ data as produced by special scientific software should be used (Wetzel et al., 2007).
The MIPAS operational data set exhibits already good quality even if some smaller problems exist as explained above. It is intended to improve this data set further by future reprocessing. Additional voluminous information about more than 20 non-operational MIPAS data products exist which have also been validated to a considerable degree.

\section{Some remarkable results}

Even if the MIPAS experiment is a novel research instrument in space the instrument operates well in general. However from March 2004 till fall 2007 the measurements are not continuous. The data have been used to retrieve operationally the temperature and six key species as well as a large number of other parameters with non-operational research algorithms. Already now, a large number of novel results in various research fields have been published in peer-reviewed journals and many more results are expected in the coming years. In the following only a selection of results is presented.

\subsection{Temperature distribution and line-of-sight pointing re- trieval}

In the operational processing temperature and pressure are retrieved from $\mathrm{CO}_{2}$ spectral signatures assuming hydrostatic equilibrium (Ridolfi et al., 2000). Total temperature errors as a function of height are estimated by Oxford University. The values are about $1.2 \mathrm{~K}$ at $12 \mathrm{~km}$ and about $2.5 \mathrm{~K}$ at $60 \mathrm{~km}$ altitude (see http://www.atm.ox.ac.uk/group/mipas/err/). The results of a comprehensive validation for temperature profiles are not fully consistent; they are described by Ridolfi et al. (2007, this Special Issue). The retrieved tangent pressure is the parameter to which all retrieved profiles are to be referred. The altitude increments between contiguous pressure levels are also determined and, given the reference of a known tangent altitude, an altitude scale can be reconstructed. However, these tangent altitudes are affected by the systematic error of the reference tangent altitude. Indeed, systematic errors greater than $1 \mathrm{~km}$ were observed in the altitude scale since the early MIPAS measurements (Ridolfi et al., 2002).

Within other approaches implemented in scientific processors, temperature fields are retrieved simultaneously with line of sight pointing information from $\mathrm{CO}_{2}$ spectral lines (von Clarmann et al., 2003b), while the pressure distribution is constructed assuming hydrostatic equilibrium from ECMWF information on pressure at $20 \mathrm{~km}$ altitude. Stratospheric temperatures derived by this approach have been validated to be consistent with meteorological analyses, radiosondes, and other satellite instruments within about $3 \mathrm{~K}$ at $50 \mathrm{~km}$ and better than $1.5 \mathrm{~K}$ at altitudes in the lower stratosphere (Wang et al., 2005a). Engineering information on line of sight pointing has been found to be biased, showing an annual cycle and latitudinal variation when compared to 


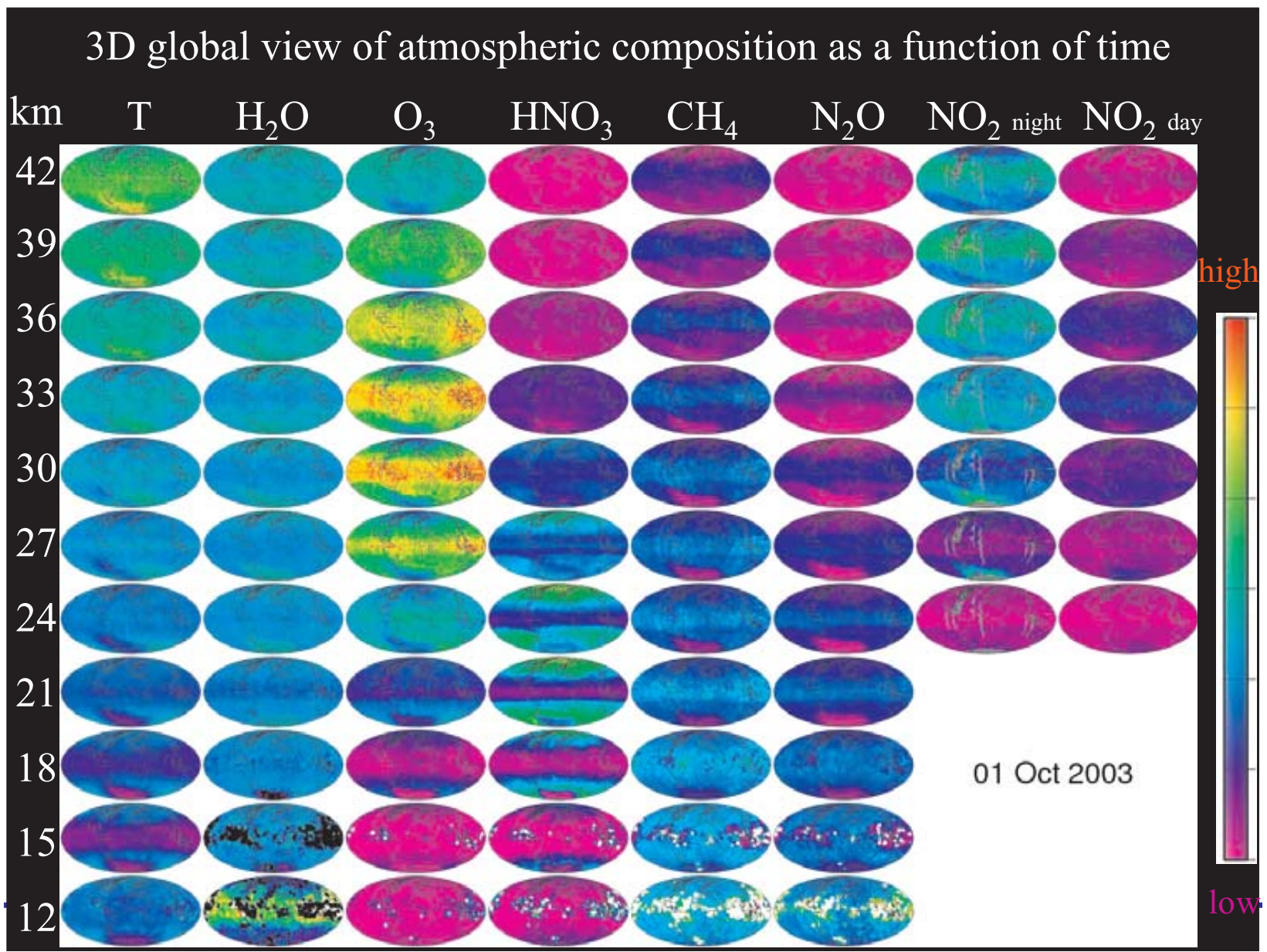

Fig. 11. Global maps of the target species as a function of altitude acquired in one day. This synopsis of the results shows the resolution and coverage of MIPAS measurements. In the case of a variable species as $\mathrm{NO}_{2}$ both a day and a night map are shown. Because of the different units and variability of the considered species qualitative units are used for this display.

retrieved line of sight pointing information. In particular the latitudinal variation could be traced back to an unrecognized bias in the roll angle of either instrument or platform of $0.05^{\circ}$ (Kiefer et al., 2007).

\subsection{Trace gas distribution as generated operationally}

MIPAS provides about 1000 profiles per day of temperature and the trace species $\mathrm{H}_{2} \mathrm{O}, \mathrm{O}_{3}, \mathrm{HNO}_{3}, \mathrm{CH}_{4}, \mathrm{~N}_{2} \mathrm{O}$, and $\mathrm{NO}_{2}$, covering the globe from pole to pole in the 6-68 km altitude range (Carli et al., 2004; see also Fig. 11). Operationally retrieved $\mathrm{HNO}_{3}$ as a proxy for $\mathrm{NO}_{\mathrm{y}}$ within the polar vortex has been used for the validation of the denitrification scheme included in SLIMCAT (Davies et al., 2006). It was possible to show, for the first time, that a sedimentation model, allowing the growth of nitric acid trihydrate (NAT) "rocks", could reproduce both the timing and spatial distribution of denitrification in the polar vortex. Assimilation schemes as such operated at University of Cologne (Schwinger et al., 2005) or
British Atmospheric Data Centre (Juckes, 2006) make extensive use of the close-to-complete spatial and temporal coverage provided by the operational MIPAS data set. Further, $\mathrm{NO}_{2}$ and $\mathrm{HNO}_{3}$ over the polar caps in Arctic and Antarctic winters from 2002 to 2004 were studied to identify long-term downward transport of mesospheric/lower thermospheric air into the stratosphere and compare it to the effects of severe solar storm events in October/November 2003 (see also following sections).

\section{$8.3 \quad \mathrm{NO}_{\mathrm{y}}$ family}

MIPAS is particularly capable of providing the total $\mathrm{NO}_{\mathrm{y}}$ budget in the stratosphere $\left(\mathrm{HNO}_{3}, \mathrm{NO}, \mathrm{NO}_{2}, \mathrm{ClONO}_{2}\right.$, $\mathrm{N}_{2} \mathrm{O}_{5}, \mathrm{HO}_{2} \mathrm{NO}_{2}$, (PAN); not observable are $\mathrm{NO}_{3}$ and $\mathrm{BrONO}_{2}$ ). For this reason, MIPAS observations are well suited to study the processes related to $\mathrm{NO}_{\mathrm{y}}$ partitioning and denitrification in polar vortices. For $\mathrm{NO}$ and $\mathrm{NO}_{2}$, sophisticated retrieval approaches taking into account Non-LTE 
effects have been developed (Funke et al., 2005a). The EC project TOPOZ-III (Kouker, 2005) was dedicated to the analysis and comparison of various chemical transport models regarding their ability to model $\mathrm{NO}_{\mathrm{y}}$ partitioning. Both the Antarctic winter 2002 and Arctic winter 2002/2003 were studied. The vortex build-up and break-up were reproduced well in all models as a result of the well-described short-term dynamics in the ECMWF analyses. Due to different microphysical parameterizations in the various models, however, the onset and fate of Polar Stratospheric Clouds (PSCs) differed strongly. A common feature of the models compared to the MIPAS measurements was the underestimation of maximum $\mathrm{HNO}_{3}$ values in air parcels which had not been affected by heterogeneous processes in the polar vortices.

\subsection{Further trace species}

Besides the near-to-complete $\mathrm{NO}_{\mathrm{y}}$ family, MIPAS spectra provide information on numerous further trace species. As a contribution to closure of the sulphur budget of the atmosphere, $\mathrm{SO}_{2}$ in volcanic plumes (Burgess et al., 2004a), OCS (Burgess et al., 2004a), and $\mathrm{SF}_{6}$ in the troposphere to lower stratosphere were derived (Burgess et al., 2004b, 2006a). Burgess et al. showed that $\mathrm{SF}_{6}$ global mean distributions provide information on inter-annual and seasonal variability, inter-hemispheric differences, as well as a tropospheric trend in accordance to ground-based in situ $\mathrm{SF}_{6}$ measurements, although the derived absolute MIPAS $\mathrm{SF}_{6}$ values were biased low. Stiller et al. (2007a) demonstrated that a global set for 19 months of the mean age of stratospheric air can be derived from MIPAS $\mathrm{SF}_{6}$ which is very consistent with model calculations of the global meridional circulation. Comparison between model and observations allows to estimate the strength of the mesospheric sink of $\mathrm{SF}_{6}$ and the atmospheric lifetime of this greenhouse gas.

An interesting field in stratospheric research still is the quantitative understanding of the coupling of the $\mathrm{HO}_{\mathrm{x}}, \mathrm{NO}_{\mathrm{x}}$, and $\mathrm{ClOx}$ catalytic cycles involved in ozone destruction. MIPAS is able to contribute also to this topic. Global distributions of $\mathrm{H}_{2} \mathrm{O}_{2}$ were derived by Papandrea et al. (2005) for December 2002, and March, June, and September 2003 although the single $\mathrm{H}_{2} \mathrm{O}_{2}$ signatures never exceed the Noise Equivalent Spectral Radiance (NESR) of the MIPAS measurements (explanation see below). The retrieved $\mathrm{H}_{2} \mathrm{O}_{2}$ values showed an equatorial mid-stratospheric peak, enhanced values in the Antarctic spring time lower stratosphere, and diurnal and seasonal variability and were in qualitative agreement with expected photochemistry. Von Clarmann et al. $(2005,2006 \mathrm{a})$ demonstrated that it is possible, similar to the $\mathrm{H}_{2} \mathrm{O}_{2}$ retrievals, to retrieve global distributions of $\mathrm{HOCl}$ from MIPAS data, although noise equivalent spectral radiance is about a factor of 10 larger than the signal of a typical single $\mathrm{HOCl}$ transition, by using redundant information of $2225 \mathrm{HOCl}$ spectral lines. $\mathrm{HOCl}$ mixing ratios were found to peak in non-polar regions at 35 to $36 \mathrm{~km}$ al-

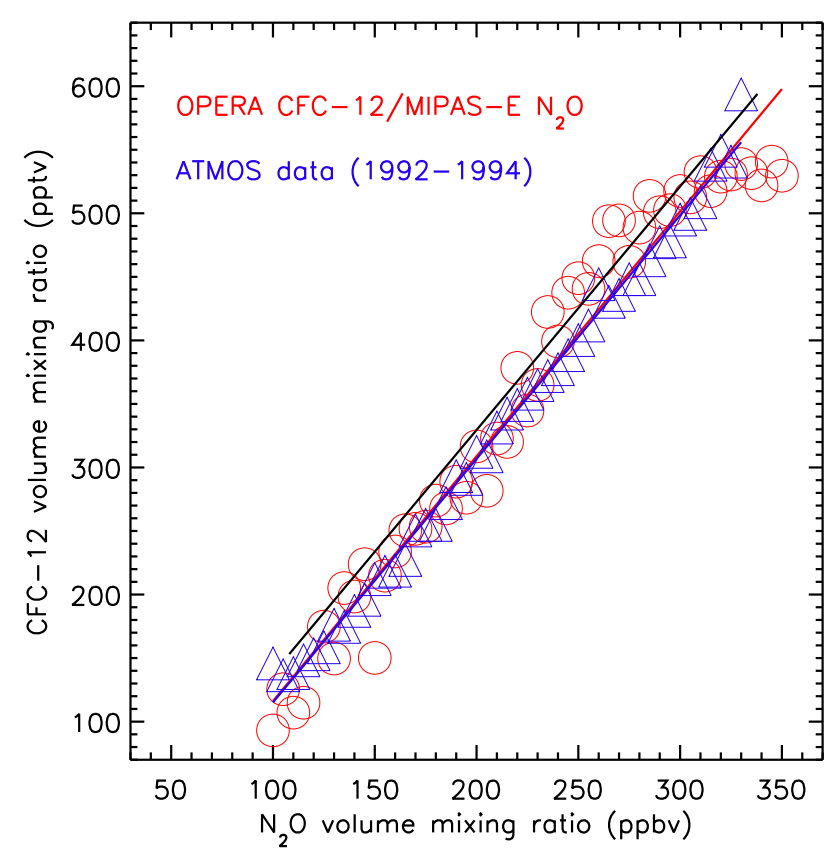

Fig. 12. ATMOS 1992-1994 (blue open triangles) and MIPAS-E 2003 (red open circles) upper tropospheric and lower stratospheric CFC-12 volume mixing ratios vs. $\mathrm{N}_{2} \mathrm{O}$. Open triangles and open squares display averages in 5 ppbv $\mathrm{N}_{2} \mathrm{O}$ intervals. The solid lines represent fits to the ATMOS (blue) and MIPAS-E (red) data. ATMOS data are only plotted where $\mathrm{N}_{2} \mathrm{O}$ vmrs exceed 100 ppbv. The solid black line shows a prediction of stratospheric CFC-12 and $\mathrm{N}_{2} \mathrm{O}$ levels based on the ATMOS data and global average trends derived from surface ESRL station data; a lower stratospheric age of air of two years (Andrews et al., 2001) was assumed and the growth rate of CFC-12 in the troposphere between 1991 and 2001 was 38 pptv from the ESRL data. (Figure taken from Moore et al., 2005).

titude, show the typical diurnal variation and are, after trend correction, consistent with former balloon-borne FIR measurements. In Antarctic polar vortex measurements, there is indication for heterogeneously produced $\mathrm{HOCl}$. After severe solar proton events, $\mathrm{HOCl}$ in the upper polar stratosphere was also found to be enhanced (see below). $\mathrm{HO}_{2} \mathrm{NO}_{2}$ is of particular interest for stratospheric chemistry since it couples the $\mathrm{NO}_{\mathrm{x}}$ and $\mathrm{HO}_{\mathrm{x}}$ catalytic cycles. Stiller et al. (2007b) provided global distributions for $\mathrm{HO}_{2} \mathrm{NO}_{2}$ from the upper troposphere to the stratosphere, and demonstrated its seasonal and diurnal variability, agreement with state-of-the-art chemical models, and the consistency to previous balloon-borne observations. Funke et al. (2006) demonstrated the feasibility to retrieve $\mathrm{CO}$ from the upper troposphere up to the mesosphere/lower thermosphere under appropriate consideration of Non-LTE effects.

Further useful species within atmospheric studies are tracers which are often used for analysis of vertical and horizontal transport processes. CFC-11 and CFC-12 have been 

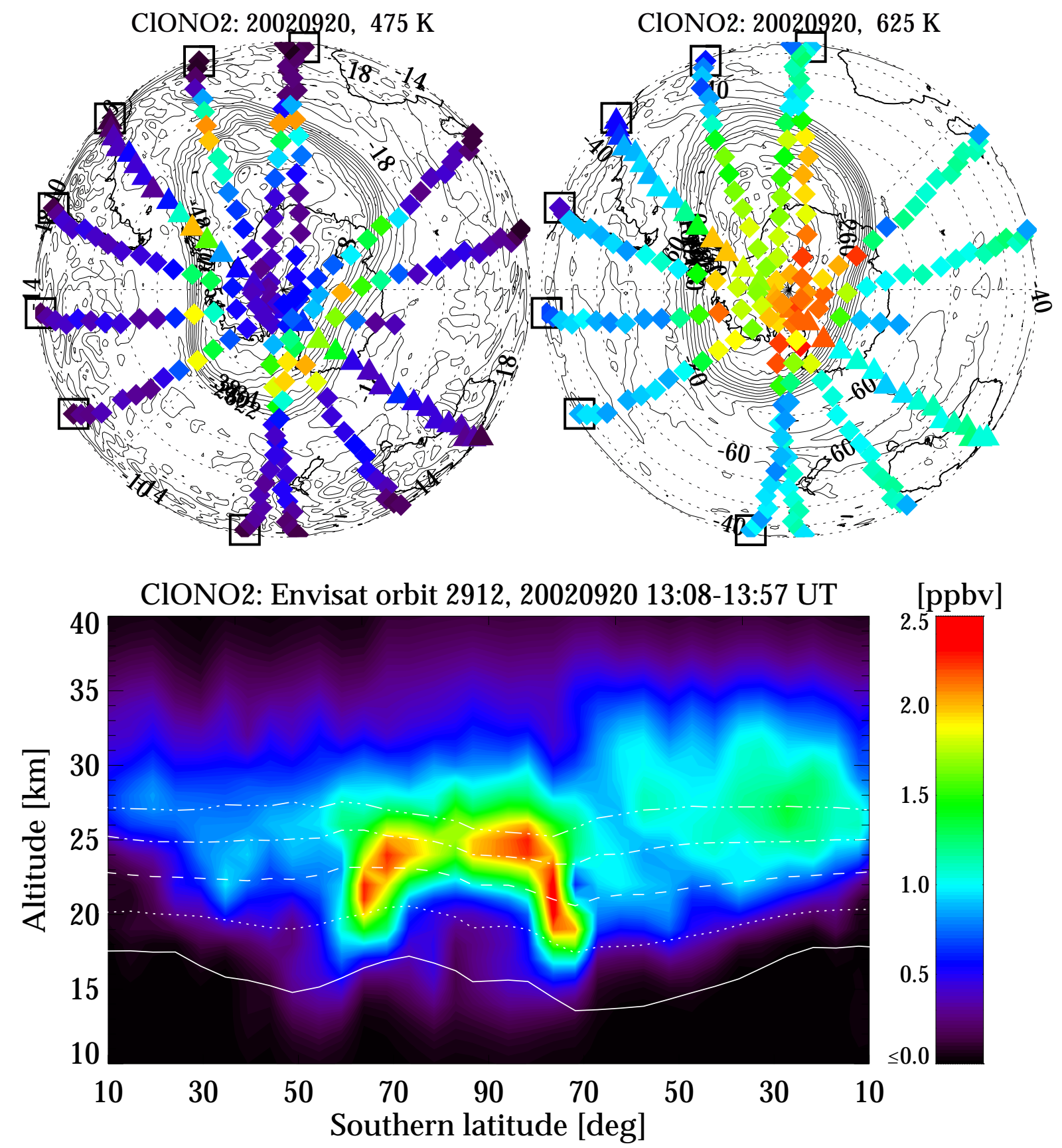

Fig. 13. $\mathrm{ClONO}_{2}$ distribution from MIPAS on 20 September 2002 for the $475 \mathrm{~K}$ (top left) and $625 \mathrm{~K}$ (top right) potential temperature surface, and at an altitude-latitude cross-section along orbit 2912 (bottom), respectively. The underlying contour lines in the top figures show potential vorticity. White lines in the bottom figure indicate potential temperature levels (figure taken from Höpfner et al., 2004a).

derived by various groups for this purpose (Hoffmann et al., 2005; Moore et al., 2006, Moore, 2005). Errors have been shown to be less than $10 \%$ for both gases between $100 \mathrm{hPa}$ and $10 \mathrm{hPa}$, except near $10 \mathrm{hPa}$ in polar winter, which is comparable to errors for $\mathrm{N}_{2} \mathrm{O}$. The MIPAS measurements therefore provide the capability to study dynamical processes with multiple tracers. Correlations between CFC-12 and
$\mathrm{N}_{2} \mathrm{O}$ have been demonstrated to be excellent in the lower stratosphere (Moore et al., 2005) as shown in Fig. 12 where the agreement between ATMOS data, adjusted for trends in CFC-12 and $\mathrm{N}_{2} \mathrm{O}$, and MIPAS data is of the order of 5\%. 


\subsection{Antarctic major warming event in September 2002}

MIPAS data were extensively used to study the unusual Antarctic major stratospheric warming in September 2002. Höpfner et al. (2004a) reported on MIPAS observations of Antarctic stratospheric chlorine nitrate recovery during Austral spring 2002 (Fig. 13). They showed that after evaporation of the last PSCs a strong increase of $\mathrm{ClONO}_{2}$ together with a simultaneous decrease of $\mathrm{ClO}$ indicated the conversion of activated chlorine into its reservoir species. Chlorine nitrate values peaked on 25 September and decreased afterwards, the chlorine being transferred into $\mathrm{HCl}$ as confirmed by HALOE observations. While this behaviour was, in general, not unusual compared to previous Antarctic winters, the levels of $\mathrm{ClONO}_{2}$ reached were extraordinarily high due to mixing of $\mathrm{NO}_{\mathrm{x}}$ rich air into the upper stratospheric vortex during this major warming event. Simultaneously, $\mathrm{ClO}$ distributions were retrieved from MIPAS spectral data (Glatthor et al., 2004). At the dayside of the Antarctic polar vortex, typical $\mathrm{ClO}$ volume mixing ratios of 1 to $2 \mathrm{ppbv}$ in the lower part of the polar vortex were observed from 8 till 23 September 2002, increasing up to 17 September and decreasing rapidly afterwards. Comparisons to observations of the MLS instrument on board the UARS satellite between 1992 and 1997 showed, in general, a similar temporal development, although providing evidence that the $\mathrm{ClO}$ deactivation was somewhat slower in those days. The warming event obviously happened too late to lead to a premature chlorine deactivation significantly different from previous Antarctic winters.

The same major warming event brought $\mathrm{NO}_{\mathrm{x}}$ and $\mathrm{O}_{3}$ rich air masses from lower latitudes near to the pole where the temperature was increased strongly by the major warming event. Formation of $\mathrm{N}_{2} \mathrm{O}_{5}$ up to unusually high values of $4.4 \mathrm{ppbv}$ at 32 to $37 \mathrm{~km}$ altitude in the dark was triggered by these preconditions (Mengistu Tsidu et al., 2004). Inside the polar vortex denitrification took place as long as PSCs were present, leading, together with quasi-horizontal mixing, to a total $\mathrm{NO}_{\mathrm{y}}$ deficit of $12.5 \mathrm{ppbv}$ (Mengistu Tsidu et al., 2005). During the major warming event the vortex boundary in the upper stratosphere became considerably permeable for midlatitudinal air, leading to a re-nitrification above $600 \mathrm{~K}$ potential temperature level of up to $5 \mathrm{ppbv}$. The $\mathrm{NO}_{\mathrm{y}}$ partitioning before and during the major warming event was neither constant in time nor in space over the polar vortex due to the heavily varying illumination situations, mixing across the vortex boundary, and high variability in temperature.

Mixing processes across the vortex boundary and ozone depletion in the lower part of the stratosphere were studied by Glatthor et al. (2005) on basis of distributions of $\mathrm{CH}_{4}, \mathrm{~N}_{2} \mathrm{O}$, CFC-11, and $\mathrm{O}_{3}$. They found that mixing into the vortex had already taken place during episodes of minor warmings before the vortex break-up event; the mixing line derived from $\mathrm{CH}_{4}-\mathrm{N}_{2} \mathrm{O}$ correlations was already flat at the beginning of the major warming so that no further mixing into the vortex could be deduced. Mixing out of the vortex, however, clearly took place above $400 \mathrm{~K}$ during the major warming event. An ongoing slight ozone loss in the order of 0.4 ppmv below $500 \mathrm{~K}$ during the major warming was derived from ozoneCFC-11 correlations. MIPAS ozone data were further used by Feng et al. (2005) to validate their model calculations of ozone loss during the Antarctic winter 2002.

The dynamics of the vortex break-up during the major warming event was studied by Wang et al. (2005b) on the basis of MIPAS ozone and temperature distributions. They analyzed the daily temperature and ozone fields by decomposition of the differences to the means into their harmonic expansions. They found that the major warming started with increased planetary wave 1 activity around 21 September. A striking feature was the presence of wave 3 variations before and after the polar vortex split on 21 and 26 September. Finally, around 27 September, wave 1 and 3 amplitudes were reduced, but those of wave 2 significantly increased. These observations strongly support model calculations (Manney et al., 2005) which indicated that wave number 3 forcing was needed to generate the observed major warming.

\subsection{Properties of Polar Stratospheric Clouds}

The MIPAS instrument has provided a unique capability for observing Polar Stratospheric Clouds (PSCs) due to its pole to pole coverage combined with wide spectral coverage and high spectral resolution. Simple cloud detection can be applied to PSCs, as described by Spang et al. (2005a), and provides a valuable, easily processed product named CIA (cloud index from MIPAS channel A). This methodology was used to good effect in the Vintersol campaign of 2002/2003 in which PSC observations, PSC model predictions, and chemical models (assimilating MIPAS observations) were employed in near real time to observe and predict the evolution of polar ozone. Spang et al. (2005a) validated the detection of PSCs against satellite and lidar measurements and demonstrated that CI-A with a threshold of 4.0 could be used to track the evolution of PSCs throughout a winter period. Spang et al. (2005b) extended this analysis to Antarctic winter 2003 and showed the differing characteristics of Antarctic versus Arctic winters with much stronger PSC intensities and more dramatic decline in cloud top altitude through the winter season in the Antarctic compared to the Arctic. The uncertainty in detection increases as CI-A increases, nonetheless Kramer (2004) showed that a threshold of 5.0 can be used for PSC detection (albeit of very low infrared opacity) in the Arctic and Antarctic at altitudes between 15 and $24 \mathrm{~km}$.

Microphysical as well as chemical composition of polar stratospheric clouds can be derived from their spectral signature, provided that scattering by PSC particles is appropriately taken into account (Höpfner et al., 2002). Spang and Remedios (2003) had shown empirically from CRISTA observations that a prominent spectral band near $820 \mathrm{~cm}^{-1}$ 
which could be distinguished in spectrally resolved infrared measurements of PSCs was probably due to nitric acid trihydrate (NAT). This inferred composition was spectroscopically proven by Höpfner et al. (2006a). Spang et al. (2005a, 2005b) applied the observation of the NAT feature to MIPAS data using simple colour ratio methods to distinguish NAT particles (of mean radius $<2 \mu \mathrm{m}$ ) from supercooled ternary solution (STS) particles and optically thick clouds (most likely ice). Höpfner et al. (2006a) extended this approach further to distinguish between the chemical composition of PSCs - ice clouds [NAT, NAD (Nitric Acid Dihydrate)] or STS clouds - by modelling their spectral signatures on basis of the refractive indices for various chemical compositions and a single scattering approach for a spherical atmosphere. Inclusion of a Mie model into the radiative transfer calculation, also providing derivatives with respect to the Mie model parameters - aerosol size distributions and chemical composition - allowed retrieval of particle parameters directly from the spectral radiances (Höpfner et al., 2002, 2004b). The chemical composition and particle size distribution as retrieved for observed PSCs during the Antarctic winter 2003 was compared and validated against ground-based LIDAR observations. In particular, it was demonstrated that the prominent $820 \mathrm{~cm}^{-1}$ feature of type 1a PSCs in MIPAS spectra could be best fitted by the application of refractive indices of $\beta$-NAT; MIPAS observations without this prominent signature could be identified as ice and STS-PSCs. No indication for the appearance of NAD in Antarctic winter 2003 was found. Further, with the help of full radiative transfer calculations, the empirical colour ratio method developed on basis of CRISTA data (Spang and Remedios, 2003) has been described quantitatively and confirmed as being able to distinguish between NAT, ice, and STS PSCs (Höpfner et al., 2006a). Based on these techniques, Höpfner et al. (2006b) discovered the evolution of a NAT belt around Antarctica in austral winter 2003, the initial appearance of which was triggered by mountain waves over the Antarctic peninsula and Ellsworth Island in early June (Fig. 14). By taking into account the mesoscale temperature distributions during the mountain wave event, the appearance and evolution of the NAT belt could successfully be modelled.

8.7 Dynamics of the middle atmosphere and downward transport of $\mathrm{NO}_{\mathrm{x}}$

Mesospheric air often subsides in polar winter and intrusion of $\mathrm{NO}_{\mathrm{x}}$ into the stratosphere is observed. During the MIPAS high spectral resolution measurement period, several episodes of severe subsidence were observed in each Arctic and Antarctic winter. The deposition of high $\mathrm{NO}_{\mathrm{x}}$ amounts in the stratosphere, however, was considerably variable. Funke et al. (2005b) analyzed the Antarctic winter 2003 and compared it to the Arctic winter 2002/2003. During Austral winter 2003, long-term continuous transport of mesospheric air into the stratosphere was detected on basis of $\mathrm{CO}$ distribu- tions which are a suitable tracer for mesospheric air. Along with high amounts of $\mathrm{CO}$, enormous burdens of $\mathrm{NO}_{\mathrm{x}}$ were transported from the upper atmosphere into the stratosphere over 2 months (Fig. 15). This was possible due to the dynamical situation in this winter which provided subsidence in the non-illuminated part of the vortex, so that the mesospheric barrier for $\mathrm{NO}_{\mathrm{x}}$ downward transport due to $\mathrm{NO}_{\mathrm{x}}$ photolysis was not active. The $\mathrm{NO}_{\mathrm{x}}$ input from the upper atmosphere into the stratospheric polar vortex amounted to 2.4 GMoles which is about $9 \%$ of the burden due to $\mathrm{N}_{2} \mathrm{O}$ photolysis. The continuous intrusion of $\mathrm{NO}_{\mathrm{x}}$, reaching up to $100 \mathrm{ppbv}$ in the upper stratosphere, led, via formation of $\mathrm{N}_{2} \mathrm{O}_{5}$ and water ion cluster chemistry, to the formation of a second $\mathrm{HNO}_{3}$ maximum at altitudes between 35 and $45 \mathrm{~km}$ with maximum vmrs of 14 ppbv (Stiller et al., 2005). Although downward transport of mesospheric air as low down as $25 \mathrm{~km}$ also happened in the Arctic winter 2002/2003, the deposition of $\mathrm{NO}_{\mathrm{x}}$ rich air was considerably lower during this winter (Funke et al., 2005b; Konopka et al., 2007). This was due to a major stratospheric warming in midwinter which interrupted the downward transport, diluted the vortex air into midlatitudes, and led to conditions without significant subsidence afterwards. The Arctic vortex in 2003/2004, in contrast, provided again favorable conditions for subsidence of mesospheric air into the stratosphere, however, this time, after a major warming that had taken place at the end of December. The vortex recovered during the first weeks in January, and developed to an extraordinary strong vortex (Manney et al., 2005) which produced severe subsidence. The $\mathrm{NO}_{\mathrm{x}}$ amounts transported from above and measured in the upper stratosphere by MIPAS (López-Puertas et al., 2005a, 2007a) and other instruments (Rinsland et al., 2005a, Randall et al., 2005) were the highest reported until then, with values up to $1 \mathrm{ppmv}$ in the upper stratosphere. From MIPAS- $\mathrm{CH}_{4}$ distributions it became obvious that rapid downward transport, concentrated on a very narrow area under dark polar winter conditions, was the cause for the high $\mathrm{NO}_{\mathrm{x}}$ amounts in the stratosphere (López-Puertas et al., 2007a).

8.8 Trace species and cirrus clouds in the upper troposphere

The investigation of the upper troposphere was not a main objective of the MIPAS instrument. However, vertical scans to low altitudes and the utilization of the cloud filtering approach has provided valuable data relevant to the monitoring and distribution of climate gases, the partitioning of sulphur compounds, and the understanding of ozone chemistry and pollution transport (by observing nitric acid and organic compounds). The MIPAS mission yields measurements on both anthropogenic greenhouse gases, such as $\mathrm{CH}_{4}, \mathrm{~N}_{2} \mathrm{O}$, and $(\mathrm{H}) \mathrm{CFCs}$, and natural greenhouses gases such as $\mathrm{H}_{2} \mathrm{O}$ and $\mathrm{O}_{3}$. UTLS CFC-11 and CFC-12 profiles derived from MIPAS have been validated by comparison with balloonborne and airborne in-situ observations (Baehr et al., 2005). 

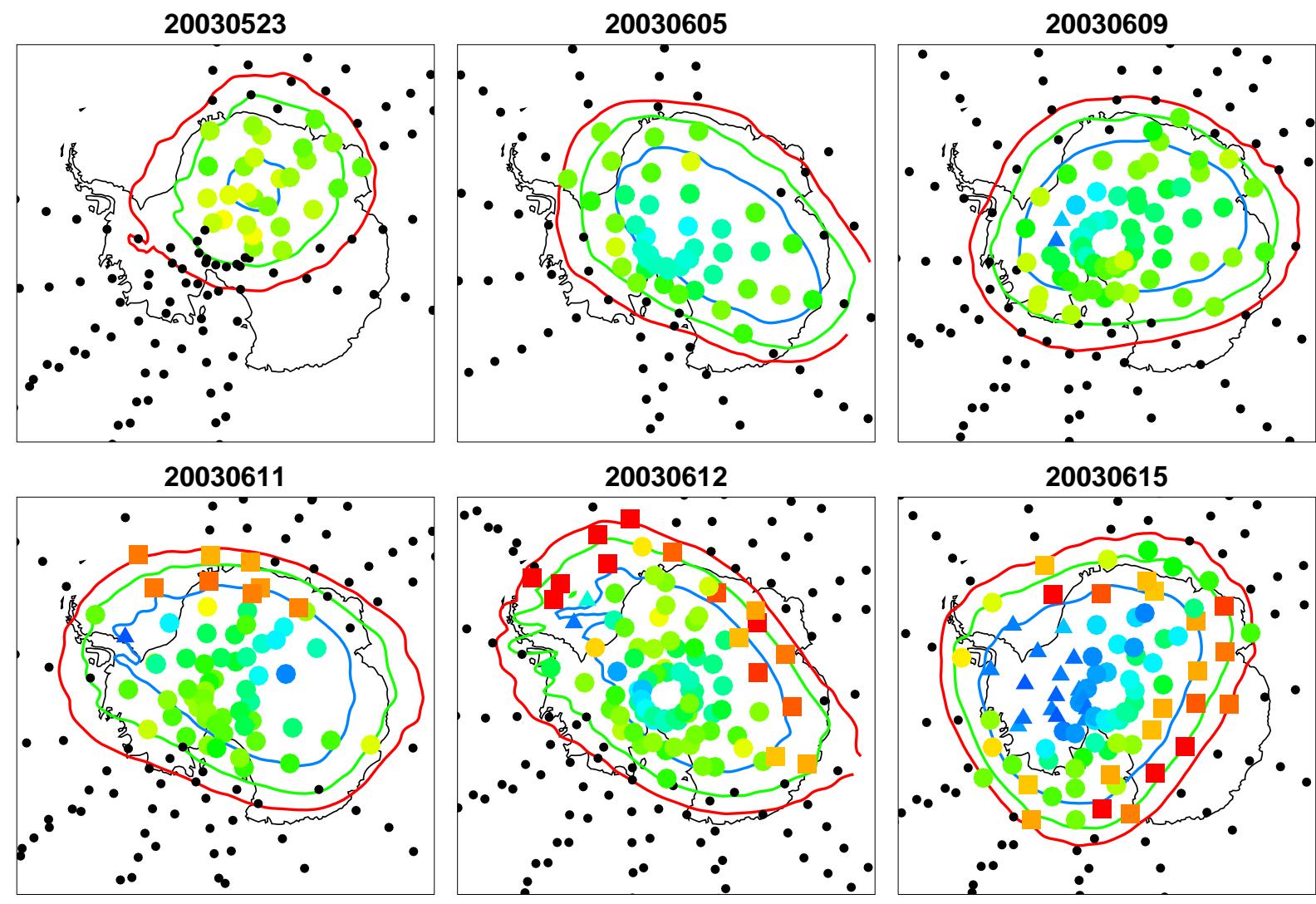

Fig. 14. Distribution of PSC types derived from MIPAS measurements at tangent altitudes corresponding to potential temperatures around $490 \mathrm{~K}$, i.e. around $21 \mathrm{~km}$ altitude. The colour scale of the data points is defined as follows: red/orange squares are NAT particles with radii $<3 \mu \mathrm{m}$, blue triangles are ice, and blue-green circles probably STS, but could also be NAT particles with radii $>3 \mu \mathrm{m}$ or thin ice clouds. The contour lines are based on ECMWF analyses and enclose the ice existence region in blue, the STS region in green and the NAT region in red. Black dots are PSC-free observations (Figure taken from Höpfner et al., 2006b).

After validation, CFC- 11 has been used to derive $\mathrm{Br}_{y}$ close to the tropopause according to an empirical correlation proposed by Wamsley et al. (1998) and to compare this to $\mathrm{BrO}$ derived from SCIAMACHY (Sinnhuber et al., 2005). HCFC-22 has been retrieved from MIPAS measurements at altitudes down to $9 \mathrm{~km}$ with systematic and random errors of less than 10\% (Moore et al., 2005). Organic compounds are an important component of the troposphere whose concentrations illustrate large-scale influences such as biomass burning, industrial pollution, and convective uplift. The MIPAS instrument has observed signatures of gases such as ethane which were expected from other spaceborne observations (e.g. Rinsland et al., 1998; Rinsland et al., 2005b), but has also provided the first spaceborne measurements of peroxyacetyl nitrate (Allen, 2005; Glatthor et al., 2006) and acetone (Remedios et al., 2006), and the first observations of the spectral signature of formic acid from a satellite instrument (Waterfall et al., 2004) (Fig. 16). Allen (2005) showed clear indications of enhanced mixing ratios for all these gases in the vicinity of the East China Sea compared to the Mediterranean, a result of anthropogenic activities such as biomass burning and industrial development. Glatthor and von Clarmann demonstrated that enhanced upper tropospheric mixing ratios of ethane, $\mathrm{PAN}, \mathrm{HNO}_{3}$, and $\mathrm{O}_{3}$ within a plume in the Southern hemisphere in autumn 2003 were due to biomass burning events in South America and Africa (Glatthor et al., 2006). $\mathrm{NH}_{3}$ plays an important role in formation of tropospheric aerosols; its retrievability from MIPAS spectra has been demonstrated by Burgess et al. (2006b). In the case of $\mathrm{CO}$, despite the overburden of the high altitude concentration, useful results have also been obtained in the upper troposphere (Belotti et al., 2006) where the comparison with MOPITT measurements (Deeter et al., 2003) shows a general agreement together with significant differences due to the complementarity of the two observation techniques (limb sounding and nadir sounding, respectively). $\mathrm{CO}$ retrievals from the mesosphere down to the middle troposphere as described by Funke et al. (2006) are in good agreement with observations of other instruments like ACEFTS or SMR/ODIN. Finally, Ewen et al. (2005) applied a reverse method Monte-Carlo transfer model together with a line-by-line model for radiative transfer to simulate multiple 

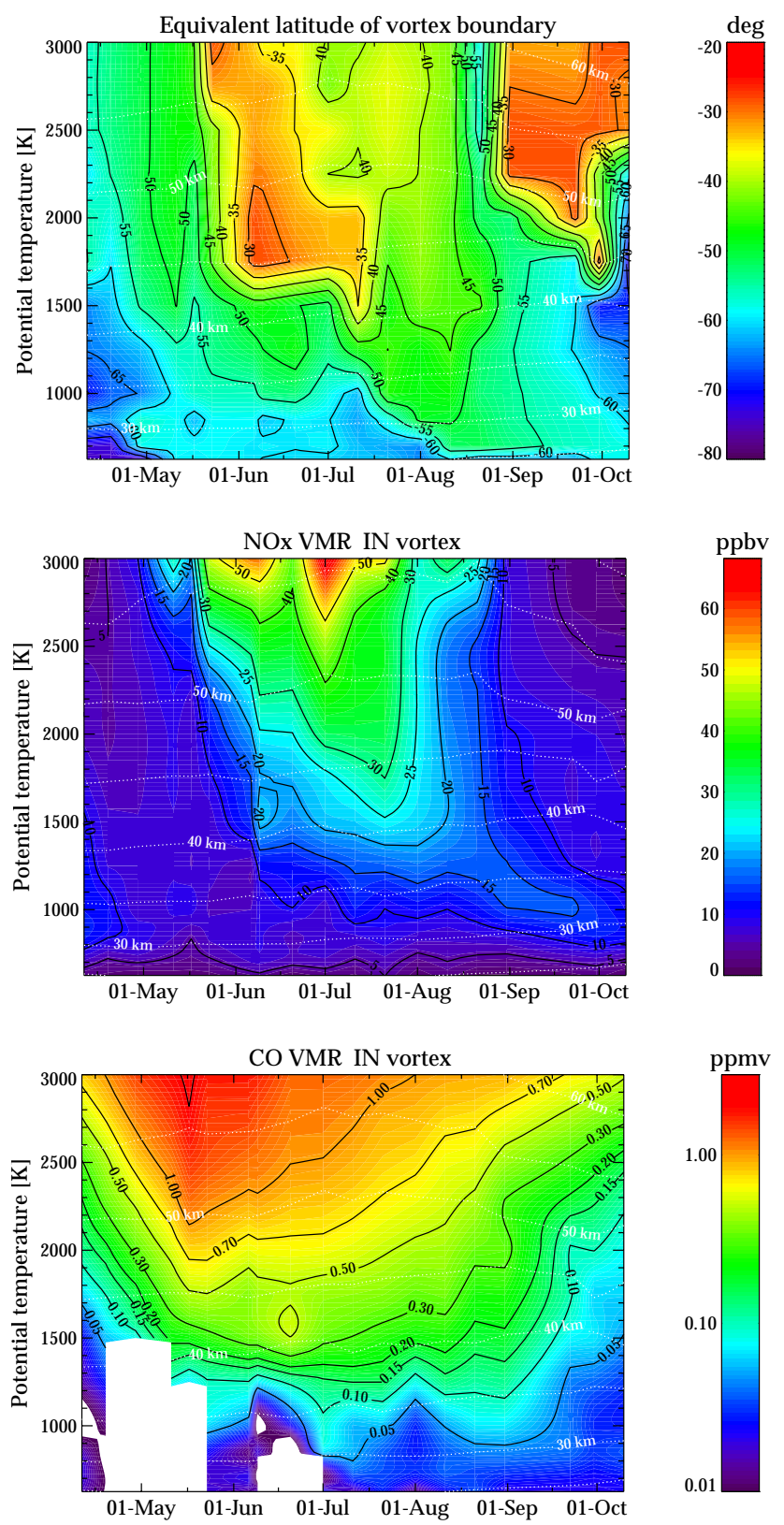

Fig. 15. Temporal evolution of the MIPAS $\mathrm{NO}_{\mathrm{x}}$ (middle) and $\mathrm{CO}$ (bottom) abundances (IMK/IAA data) averaged over the vortex core region at isentropic surfaces from $625 \mathrm{~K}$ to $3000 \mathrm{~K}$ during Antarctic winter 2003. An area-weighting factor (cosine of latitude) has been applied to $\mathrm{NO}_{\mathrm{x}}$ and $\mathrm{CO}$. White dotted lines represent geometric altitudes in $\mathrm{km}$. White regions are missing data (figure taken from Funke et al., 2005b).

scattering by ice particles in cirrus clouds. The simulations showed good agreement with MIPAS spectra of cloudy scenes; in particular, $\mathrm{H}_{2} \mathrm{O}$ absorption lines which are due to upwelling tropospheric radiation scattered into the line-ofsight by the cloud ice particles, first detected in PSC signals (Höpfner et al., 2002), can be simulated.

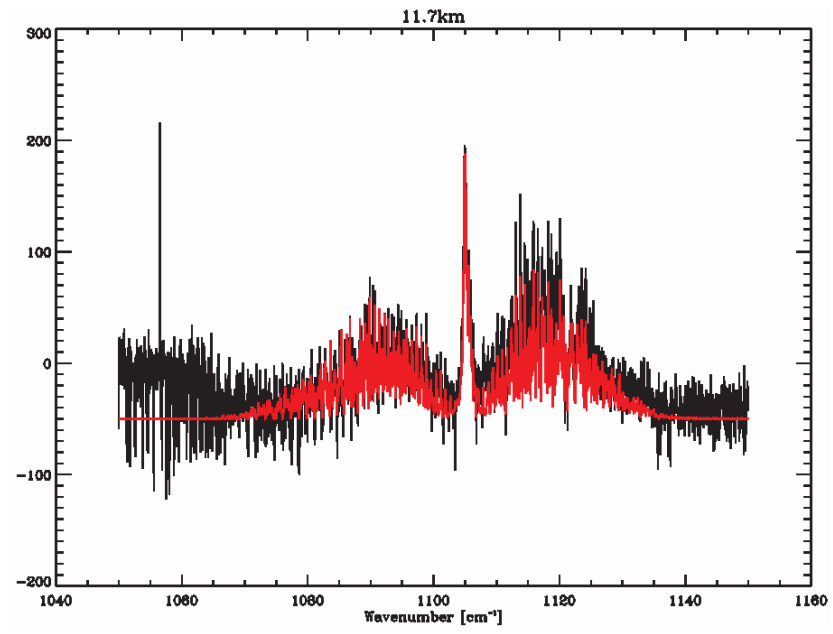

Fig. 16. Detection of formic acid by residual analysis for $1.2 \mathrm{ppbv}$ formic acid between 9 and $11 \mathrm{~km}$ over the China Sea in April 2003. Residual of MIPAS spectrum minus a forward model simulation not including formic acid (dark line) compared to the difference between forward model calculations with and without formic acid. Good agreement between measured residuals and forward model differences indicate positive detection. The noise in the formic acid spectral region is less than $30 \mathrm{nW} /\left(\mathrm{cm}^{2} \mathrm{sr} \mathrm{cm}^{-1}\right)$ respectively. (figure taken from Remedios et al., 2006; Allen, 2005).

\subsection{Stratosphere-troposphere exchange}

The details of exchange processes between troposphere and stratosphere are not yet fully understood up to now. MIPAS can contribute to this topic by the global distributions of water vapour and inert trace species in the upper troposphere and lowermost stratosphere. $\mathrm{SF}_{6}$ distributions (Burgess et al., 2004b, 2006a; Stiller et al., 2007a) allow the mean age of stratospheric air to be assessed, and thus help to validate the modelling of the Brewer-Dobson circulation in various chemistry transport models (CTMs). Transport of water vapour through the tropical tropopause can be studied in detail based on high-quality water vapour distributions from MIPAS (Milz et al., 2005). Water vapour in the tropical lowermost stratosphere in summer (JJA) showed values as low as 2.5 ppmv. In contrast, rather high water vapour vmrs of more than $6 \mathrm{ppmv}$ are found in the subtropical lowermost stratosphere around $18 \mathrm{~km}$ altitude. This hints towards differences in the water vapour transport processes through the tropopause between the tropics and subtropics.In particular, relatively high water vapour vmrs were found at about $20 \mathrm{~km}$ altitude in the Himalayan region, indicating monsoon activities to be responsible for these (Milz et al., 2005; Fig. 17). Studies on the details of transport through the tropical tropopause and related dehydration processes are supported by distributions of HDO in the troposphere and lowermost stratosphere which can also be derived from MIPAS (Steinwagner et al., 2007). 


\subsection{Influence of solar variability}

MIPAS characteristics and its global measurements mode enable us to capture, as with no other instrument before, the chemical changes in the atmosphere induced by solar proton events. MIPAS observed after the solar proton event (SPE) in October-November 2003 composition changes in the stratosphere and mesosphere in both polar regions for a large number of atmospheric species $\left(\mathrm{NO}, \mathrm{NO}_{2}, \mathrm{O}_{3}, \mathrm{HNO}_{3}\right.$, $\mathrm{N}_{2} \mathrm{O}_{5}, \mathrm{ClONO}_{2}, \mathrm{HOCl}, \mathrm{ClO}$ ) (López-Puertas et al., 2005a; ; von Clarmann et al., 2005) (Fig. 18).

MIPAS observed very large SPE-induced changes in the $\mathrm{NO}_{\mathrm{x}}$ concentrations in the stratosphere and mesosphere in both polar regions, with very large values (up to $180 \mathrm{ppbv}$ ) over the Northern polar cap and a large asymmetry between the Northern (winter) and Southern (summer) polar caps. Arctic mean polar cap $\left(>60^{\circ}\right) \mathrm{NO}_{\mathrm{x}}$ enhancements of 20 to $70 \mathrm{ppbv}$ between 40 to $60 \mathrm{~km}$ lasted for at least two weeks, while the Antarctic mean $\mathrm{NO}_{\mathrm{x}}$ enhancement was between 10 and $35 \mathrm{ppbv}$ and was halved after two weeks. Also, ozone showed depletion signatures associated with both $\mathrm{HO}_{\mathrm{x}}\left(\mathrm{H}+\mathrm{OH}+\mathrm{HO}_{2}\right)$ and $\mathrm{NO}_{\mathrm{x}}$ enhancements at different time scales from hours $\left(\mathrm{HO}_{\mathrm{x}}\right)$ to weeks $\left(\mathrm{NO}_{\mathrm{x}}\right)$. Arctic lower mesospheric (upper stratospheric) ozone was reduced by $50-70 \%$ (30-40\%) for about two weeks after the SPEs but a much smaller ozone depletion was observed over the Antarctic polar cap.

MIPAS also observed significant enhancements of other $\mathrm{NO}_{\mathrm{y}}$ components such as $\mathrm{HNO}_{3}, \mathrm{~N}_{2} \mathrm{O}_{5}$, and $\mathrm{ClONO}_{2}$ in the Northern polar stratosphere after the intense solar proton events (López-Puertas et al., 2005b). Two distinct enhancements were observed for $\mathrm{HNO}_{3}$ : an instantaneous increase of 1-2 ppbv (the origin of which is still unclear although it seems that the water vapour ion chemistry might play an important role; López-Puertas et al., 2007a) and a larger second increase of $1-5 \mathrm{ppbv}$ which started around November 10 and lasted until the end of December 2003. The second event is attributed to the enhanced $\mathrm{NO}_{\mathrm{x}}$ produced in the mesosphere during the major SPEs, which was transported downwards in the following months, partially converted to $\mathrm{N}_{2} \mathrm{O}_{5}$ in the upper stratosphere, and then finally converted to $\mathrm{HNO}_{3}$ via ion cluster reactions (Stiller et al., 2005). $\mathrm{N}_{2} \mathrm{O}_{5}$ was also observed to increase by $0.1-0.4 \mathrm{ppbv}$ one to three days after the major SPE, when the enhancement reached down to $30 \mathrm{~km}$. A second, more pronounced $\mathrm{N}_{2} \mathrm{O}_{5}$ enhancement of up to $1.2 \mathrm{ppbv}$ appeared at $40 \mathrm{~km}$ about $12-13$ days after the major SPEs. The $\mathrm{HNO}_{3}$ and $\mathrm{N}_{2} \mathrm{O}_{5}$ enhancements in the Southern hemisphere were generally much less pronounced (López-Puertas et al., 2005b).

Besides ozone and $\mathrm{NO}_{\mathrm{y}}$, MIPAS observed the impact of the SPEs on the stratospheric chlorine chemistry, for the first time ever (von Clarmann et al., 2005). An increase in ClO, $\mathrm{HOCl}$, and $\mathrm{ClONO}_{2}$ was observed, more pronounced over the Arctic winter polar cap than in the Antarctic. The $\mathrm{HOCl}$ increase was pronounced but lasted only for about 2 days

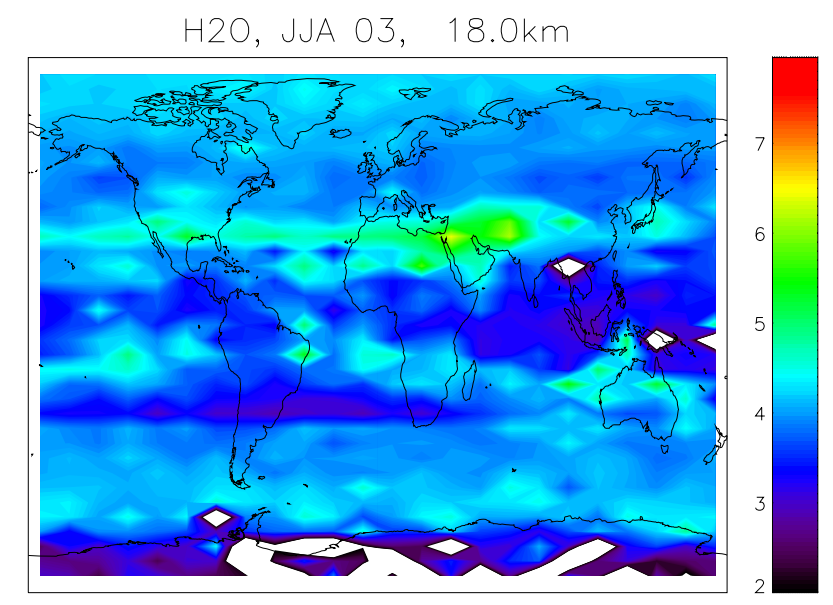

Fig. 17. Averaged water vapour distribution for $5^{\circ} \times 15^{\circ}$ latitudelongitude bins for available measurements during June, July, and August 2003. White areas indicate regions where no information is available from MIPAS (e.g., due to cloud contamination) (Figure taken from Milz et al., 2005).

after the SPEs which is explained by reaction of $\mathrm{HO}_{\mathrm{x}}$ produced locally during the SPEs with $\mathrm{HCl}$. Increased vmrs of $\mathrm{ClO}$ and $\mathrm{HOCl}$ were spatially anticorrelated indicating that missing $\mathrm{HOCl}$ production was not restricted by available $\mathrm{ClO}$. This also confirms that chlorine was provided from $\mathrm{HCl}$ for the production of $\mathrm{ClO}$ and $\mathrm{HOCl}$. The increase of $\mathrm{ClONO}_{2}$, starting later but lasting longer than the other chlorine species, indicates that a persistent change in the chlorine reservoir partitioning between $\mathrm{HCl}$ and $\mathrm{ClONO}_{2}$ was caused by the SPEs.

\subsection{Observation of Non-LTE effects}

MIPAS has several advantages which makes it a very suitable instrument for studying the atmospheric non-local thermodynamic equilibrium (Non-LTE) emissions. Reasons are its wide spectral coverage, allowing for measuring emissions from a given compound in different spectral regions, its high spectral resolution, enabling the discrimination of emissions from different species and between different bands of the same species, and its high sensitivity allowing the emission to be measured in the upper atmosphere where Non-LTE emissions are most important. All this has led MIPAS to provide us with significant improvements in our knowledge of Non-LTE atmospheric emissions of which we include here a succinct list.

MIPAS has provided the first evidence of $\mathrm{CH}_{4}\left(v_{4}\right)$ NonLTE emissions in the mesosphere (López-Puertas et al., 2005d). Regarding the Non-LTE processes in $\mathrm{CO}_{2}$, the high spectral resolution of MIPAS has allowed to derive new collisional rates between the $\mathrm{CO}_{2} 02^{0} 1,02^{2} 1$, and $10^{0} 1$ states that emit the $4.3 \mu \mathrm{m}$ second hot bands, which differ by factors of 

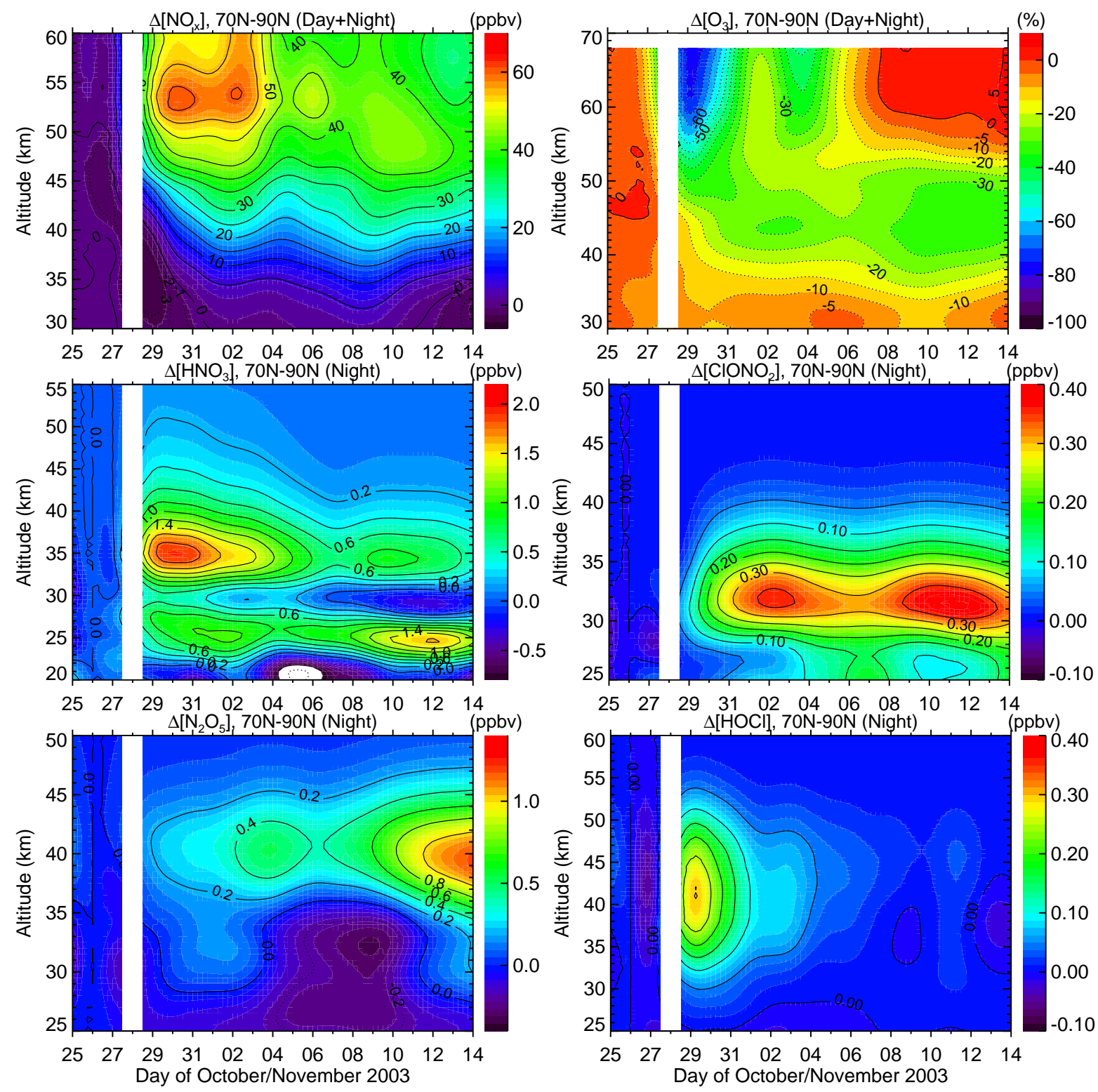

Fig. 18. Temporal evolution of $\mathrm{NO}_{\mathrm{x}}$ (top left), $\mathrm{O}_{3}$ (top right), $\mathrm{HNO}_{3}$ (middle left), $\mathrm{ClONO}_{2}$ (middle right), $\mathrm{N}_{2} \mathrm{O}_{5}$ (bottom left), and $\mathrm{HOCl}$ (bottom right) during and after the October - November 2003 solar proton events for the Southern hemisphere $\left(70^{\circ} \mathrm{S}-90^{\circ} \mathrm{S}\right)($ left panels) and Northern hemisphere $\left(70^{\circ} \mathrm{N}-90^{\circ} \mathrm{N}\right)$ (right panels) polar caps. Changes are shown relative to the mean profiles measured on 25 October 2003 (Figures taken from López-Puertas et al., 2005a $\left(\mathrm{NO}_{\mathrm{x}}\right.$ and $\left.\mathrm{O}_{3}\right)$, López-Puertas et al., 2005b $\left(\mathrm{HNO}_{3}, \mathrm{ClONO}_{2}\right.$, and $\left.\mathrm{N}_{2} \mathrm{O}_{5}\right)$, and von Clarmann et al., 2005 (HOCl)).

3.5 and 0.025 , respectively, from previously consensus values (López-Puertas et al., 2005c) (Fig. 19).

Concerning Non-LTE in $\mathrm{O}_{3}$, a preliminary analysis of MIPAS spectra in the $2080-2130 \mathrm{~cm}^{-1}$ region has shown that current Non-LTE models underestimate the MIPAS daytime radiance by a factor of $2-3$ in the $60-70 \mathrm{~km}$ region (Fig. 19). Possible reasons for this discrepancy are that $\mathrm{O}_{3}$ is formed at lower vibrational levels, and/or the collisional relaxation of the stretching $\left(v_{1}, v_{3}\right)$ is weaker (Kaufmann et al., 2006).

MIPAS has also taken remarkable spectra of $\mathrm{H}_{2} \mathrm{O}$ in the mesosphere, unequivocally demonstrating the distinct NonLTE daytime emissions from the fundamental and hot bands at $6.3 \mu \mathrm{m}$. Furthermore, it has provided the most accurate collisional rate for the relaxation of $\mathrm{H}_{2} \mathrm{O}(020)$ by $\mathrm{O}_{2}$, with a value of $1.36 \times 10^{-12} \mathrm{~cm}^{3} \mathrm{~s}^{-1}$ and a $10 \%$ uncertainty (Koukouli et al., 2006, 2007 ${ }^{1}$ ), and the rate of quenching of

\footnotetext{
${ }^{1}$ Koukouli, M. E., López-Puertas, M., Funke, B., Gil-López, G., Kaufmann, M., Milz, M., von Clarmann, T., and Stiller, G. P.: Water vapour $6.3 \mu \mathrm{m}$ non-local thermodynamic equilibrium emissions as
} 
$\mathrm{O}_{2}(\mathrm{v}=1)$ by atomic oxygen $\mathrm{O}\left({ }^{3} \mathrm{P}\right)$ measured by Kalogerakis et al. (2005) has been further confirmed.

In addition, MIPAS has given the first experimental confirmation of the Non-LTE processes exciting the $\mathrm{NO}(v=1)$ level, emitting near $5.3 \mu \mathrm{m}$, in the daytime stratosphere (Funke et al., 2005a) as proposed by Kaye and Kumer (1987). MIPAS has also resolved the NO vibrationrotation transitions for both ${ }^{2} \Pi_{1 / 2}$ and ${ }^{2} \Pi_{3 / 2}$ spin-orbit components in the $5.3 \mu \mathrm{m}$ emission in the thermosphere, both under quiescent and geomagnetically perturbed conditions. A preliminary analysis has confirmed previous spin component temperatures (Gardner et al., 2005).

MIPAS has also shed light on the puzzle about whether or not the $\mathrm{NO}_{2}\left(\mathrm{v}_{3}\right)$ levels are Non-LTE excited in the daytime stratosphere. A recent analysis has shown (Funke et al., 2005a) that the photochemical excitation of $\mathrm{NO}_{2}\left(\mathrm{v}_{3}=1-\right.$ 4) levels is a factor of 50 smaller than previously thought, leading to small Non-LTE deviations for these levels.

Finally, measurements of the Non-LTE emissions of the carbon monoxide first hot band, $\mathrm{CO}(2-1)$ near $4.7 \mu \mathrm{m}$ (Fig. 19), and of $\mathrm{N}_{2} \mathrm{O}(001)$ near $4.5 \mu \mathrm{m}$ have also been observed by MIPAS by the first time (Funke et al., 2007; LópezPuertas et al., 2007b).

\section{Conclusions and outlook}

MIPAS provides global measurements of mid-infrared spectra of high quality for the determination of temperature and more than 25 trace constituents as well as aerosol/cloud parameters during day and night. The pole-to-pole measurements allow the Arctic and the Antarctic areas to be investigated with extraordinary good data coverage.

The operational MIPAS data processing of ESA generates global distributions of temperature and six key species $\left(\mathrm{O}_{3}\right.$, $\mathrm{H}_{2} \mathrm{O}, \mathrm{CH}_{4}, \mathrm{~N}_{2} \mathrm{O}, \mathrm{NO}_{2}, \mathrm{HNO}_{3}$ ) while scientific data processing has already proven that many more trace gases can be derived from the mid-infrared spectra $\left(\mathrm{NO}, \mathrm{N}_{2} \mathrm{O}_{5}, \mathrm{HNO}_{4}\right.$, $\mathrm{ClONO}_{2}, \mathrm{ClO}, \mathrm{HOCl}, \mathrm{CO}, \mathrm{CFCs}, \mathrm{NH}_{3}, \mathrm{OCS}, \mathrm{SO}_{2}, \mathrm{SF}_{6}$, PAN, HCN, $\mathrm{C}_{2} \mathrm{H}_{6}, \mathrm{C}_{2} \mathrm{H}_{2}, \mathrm{H}_{2} \mathrm{O}_{2}, \mathrm{HDO}, \mathrm{O}_{3}$ isotopologues). Furthermore, the MIPAS broad-band spectra can be used for the determination of aerosol parameters (polluted atmosphere) and concentration, mean radius, and composition of cloud particles (PSCs, cirrus).

The data quality of MIPAS results is generally good as is shown in various articles of this ACP Special Issue and many other articles on validation of products as generated by scientific MIPAS data processors. This statement is supported by the findings of three ESA Atmospheric Chemistry Validation of ENVISAT (ACVE)-Workshops in December 2002, May 2004, and December 2006.

Many interesting results have already been achieved from MIPAS measurements. They include contributions to a better understanding of atmospheric processes like pollution of measured by MIPAS/ENVISAT, in preparation 2008.

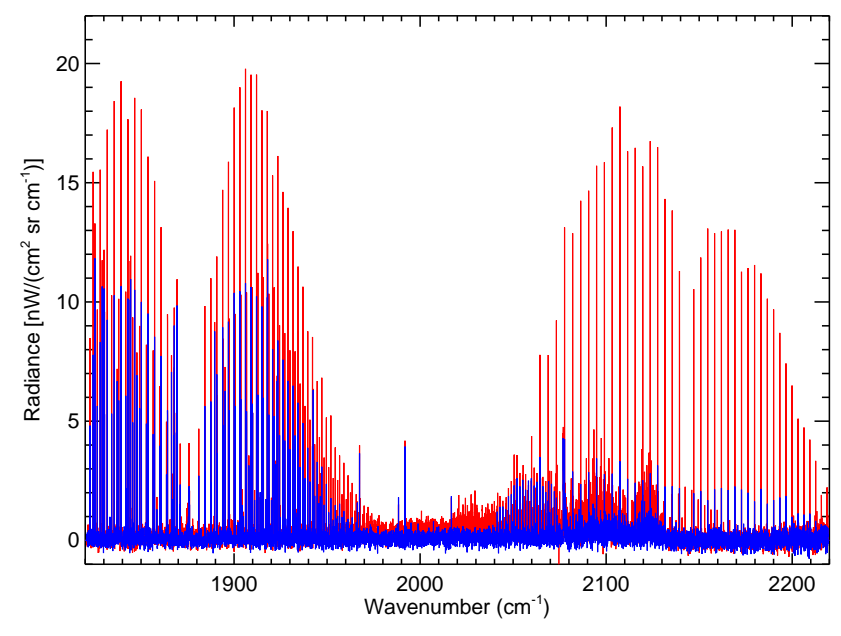

Fig. 19. MIPAS co-added spectra in band D for the upper atmosphere measurements taken on 2nd July 2002 at a tangent height of $\sim 61 \mathrm{~km}$. Daytime in red and night-time in blue. The number of co-added spectra are 95 and 94 for day and night, respectively. The mean solar zenith angle for daytime is $46^{\circ}$. The figure shows the spectral region from 1820 to $2220 \mathrm{~cm}^{-1}$ where the major atmospheric emitters in the middle atmosphere are $\mathrm{NO}, \mathrm{O}_{3}$, and $\mathrm{CO}$. The high spectral resolution of MIPAS enables to resolve the NO vibration-rotation lines from both spin-orbit components. The day/night differences in the $\mathrm{O}_{3}$ bands are clearly observed (1950$2150 \mathrm{~cm}^{-1}$ ) with larger contribution in the daytime. The contributions from the different hot bands are clearly identified around 2000 , 2030, 2060, 2090 and $2120 \mathrm{~cm}^{-1}$. The day/night differences in the $\mathrm{CO}(1-0)$ band are also very prominent $\left(2050-2220 \mathrm{~cm}^{-1}\right)$.

the upper troposphere, troposphere-stratosphere exchange, chemistry and dynamics of the stratosphere, stratospheric ozone depletion, downward transport from the mesosphere into the stratosphere, interaction between varying solar radiation and atmospheric composition, and Non-LTE. Although MIPAS was designed as an instrument for research purposes, the data have also been used by ECMWF for testing their influence on weather forecast. Due to the positive outcome ECMWF is interested in receiving MIPAS NRT data in the future.

Due to certain problems with the constant movement of the retroreflectors of the interferometer, the measurements of MIPAS have been performed with reduced spectral resolution and a reduced duty cycle since the beginning of 2005 . The spectral resolution is reduced to $40 \%$ which in turn allows for a higher spatial resolution (vertically and horizontally). The duty cycle has been reduced to about $35 \%$ in 2005. Nevertheless, MIPAS is detecting in this mode more than 500 profiles of various atmospheric parameters on average every day. More recently it has been recognized that the problems with the moving retroreflectors are decreasing. Therefore the duty cycle is increased step-by-step to currently 100\% (December 2007), following the recommendations of the MIPAS Science Team. 
A satellite instrument of similar type called TES (Tropospheric Emission Spectrometer, Beer 2006) has been developed for the American AURA mission launched in 2004. In opposition to MIPAS the measurements of TES are mainly used for studying tropospheric processes and as a consequence complementing the MIPAS results.

A new generation model of the MIPAS instrument is already in development for future missions. IMK (Institute for Meteorology and Climate Research)/Karlsruhe in cooperation with ICG (Institut für Chemie und Dynamik der Geosphäre)/Jülich has built up a FTIR with a two-dimensional detector array $(128 \times 128$ elements, FriedlVallon et al., 2006, Riese et al., 2005) in the laboratory. A corresponding instrument for aircraft application is being developed. This kind of instrument offers the chance to perform future spectral IR measurements with considerably higher spatial resolution and better coverage in the atmosphere. A similar instrument - the so-called IMIPAS (Imaging MIPAS) - has been proposed in 2006 for an ESA Earth Explorer Mission and has been selected for a currently running prephase A study. It is expected that the new IMIPAS will be able to yield essentially improved data for the investigation of mesoscale processes in the atmosphere like the stratosphere-troposphere exchange.

Acknowledgements. We gratefully acknowledge the large effort of ESA to build and operate the MIPAS experiment aboard ENVISAT and to provide MIPAS Level 1 and Level 2 data. Meteorological analyses have been provided by ECMWF. The IMK team has been supported by several EC and BMBF projects. The IAA team was supported by the Spanish project ESP2004-01556 and EC FEDER funds. The Leicester team was supported by grants from the UK Natural Environment Research Council and from ESA. Many more scientists and engineers from various institutions have contributed to the MIPAS experiment; their aid is greatly acknowledged.

Edited by: P. Hartogh

\section{References}

Allen, G.: The Infrared Remote Sensing of Peroxyacetyl Nitrate in the Upper Troposphere, Ph.D. thesis, University of Leicester, 2005.

Andrews, A. E., Boering, K. A., Dauble, B. C., Wofsy, S. C., Loewenstein, M., Jost, H., Podolske, J. R., Webster, C. R., Herman, R. L., Scott, D. C., Flesch, G. J., Moyer, E. J., Elkins, J.W., Dutton, G. S., Hurst, D. F., Moore, F. L., Ray, E. A., Romashkin, P. A., and Strahan, S. E.: Mean ages of stratospheric air derived from in situ observations of $\mathrm{CO}_{2}, \mathrm{CH}_{4}$, and $\mathrm{N}_{2} \mathrm{O}$, J. Geophys. Res., 106(D23), 32 295-32 314, 2001.

Baehr, J., Volk, C. M., Ivanova, E., Werner, A., Wetter, T., Engel, A., Haase, H.-P., Moebius, T., Schmidt, U., Stiller, G., von Clarmann, T., Glatthor, N., Kellmann, S., Grunow, K., and Levin, I.: Validation of MIPAS-ENVISAT $\mathrm{CH}_{4}, \mathrm{~N}_{2} \mathrm{O}, \mathrm{CFC}-11$, and CFC12 by Airborne in situ Observations, in: Proc. of the 2004 ENVISAT and ERS Symposium, edited by: Lacoste, H. and Ouwe- hand, L., 6-10 September 2004, Salzburg, Austria, (ESA SP572, April 2005) CD-ROM, ISBN 92-9092-883-2, ISSN 1609042X, 2005.

Beer, R., TES on the AURA Mission: Scientific Objectives, Measurements and Analysis Overview, IEEE Trans. Geosci. Remote Sensing, 44, 1102-1105, 2006.

Belotti, C., Carli, B., Ceccherini, S., Del Bianco, S., Jin, J., and Raspollini, P.: Retrieval of tropospheric Carbon Monoxide from MIPAS measurements, Proc. of the Atmospheric Science Conference- ESA ESRIN, May 2006 (http://earth.esa.int/cgi-bin/ confatmos06.pl?abstract=388, 2006.

Burgess, A. B., Grainger, R. G., and Dudhia, A.: Progress in the retrieval of sulphur species from MIPAS, ESA: ENVISAT-ERS Symposium Proceedings (SP-572), 2004a.

Burgess, A. B., Grainger, R. G., Dudhia, A., Payne, V. H., and Jay, V. H.: MIPAS Measurement of Sulphur Hexafluoride $\left(\mathrm{SF}_{6}\right)$, Geophys. Res. Lett., 31(5), L07809, doi:10.1029/2003GL019143, 2004b.

Burgess, A. B., Grainger, R. G., and Dudhia, A.: Zonal mean atmospheric distribution of sulphur hexafluoride $\left(\mathrm{SF}_{6}\right)$, Geophys. Res. Lett., 33, L07809, doi:10.1029/2005GL025410, 2006 a.

Burgess, A. B., Dudhia, A., Grainger, R. G., and Stevenson, D.: Progress in tropospheric ammonia retrieval from the MIPAS satellite instrument, Adv. Space Res., 37, 12, 2218-2221, 2006b.

Carli, B., Alpaslan, D., Carlotti, M., Castelli, E., Ceccherini, S., Dinelli, B. M., Dudhia, A., Flaud, J.-M., Höpfner, M., Jay, V., Magnani, L., Oelhaf, H., Payne, V., Piccolo, C., Prosperi, M., Raspollini, P., Remedios, J., Ridolfi, M., and Spang, R.: First results of MIPAS/ENVISAT with operational Level 2 code, Adv. Space Res., 33, 7, 1012-1019, doi:10.1016/S02731177(03)00584-2, 2004.

Carlotti, M.: Global-fit approach to the analysis of limb-scanning atmospheric measurements, Appl. Opt., 27, 3250-3254, 1988.

Carlotti, M., Dinelli, B. M., Raspollini, P., and Ridolfi, M.: Geofit approach to the analysis of limb-scanning satellite measurements, Appl. Opt., 40(12), 1872-1885, 2001.

Carlotti, M., Brizzi, G., Papandrea, E., Prevedelli, M., Ridolfi, M., Dinelli, B. M., and Magnani, L.: GMTR: two-dimensional multitarget retrieval model for MIPAS/ENVISAT observations, Appl. Opt., 45(4), 716-727, 2006.

Cortesi, U., Lambert, J. C., De Clercq, C., Bianchini, G., Blumenstock, T, Bracher, A., Castelli, E., Catoire, V., Chance, K. V., De Mazière, M., Demoulin, P., Godin-Beekmann, S., Jones, N., Jucks, K., Keim, C., Kerzenmacher, T., Kuellmann, H., Kuttippurath, J., Iarlori, M., Liu, G, Y., Liu, Y., McDermid, I. S., Meijer, Y. J., Mencaraglia, F., Mikuteit, S., Oelhaf, H., Piccolo, C., Pirre, M., Raspollini, P., Ravegnani, F., Reburn, W. J., Redaelli, G., Remedios, J. J., Sembhi, H., Smale, D., Steck, T., Taddei, A., Varotsos, C., Vigouroux, C., Waterfall, A., Wetzel, G, and Wood, S.: Geophysical validation of MIPAS-ENVISAT operational ozone data, Atmos. Chem. Phys., 7, 4807-4867, 2007, http://www.atmos-chem-phys.net/7/4807/2007/.

Davies, S., Mann, G. W., Carslaw, K. S., Chipperfield, M. P., Remedios, J. J., Allen, G., Waterfall, A. M., Spang, R., and Toon, G. C.: Testing our understanding of Arctic denitrification using MIPAS-E satellite measurements in winter 2002/2003, Atmos. Chem. Phys., 6, 3149-3161, availible at:http://direct.sref. org/1680-7324/acp/2006-6-3149, 2006.

Deeter, M. N., Emmons, L. K., Francis, G. L., Edwards, D. P., Gille, 
J. C., Warner, J. X., Khattatov, B., Ziskin, D., Lamarque, J.-F., Ho, S.-P., Yudin, V., Attié, J.-L., Packman, D., Chen, J., Mao, D., and Drummond, J. R.: Operational carbon monoxide retrieval algorithm and selected results for the MOPITT instrument, J. Geophys. Res., 108(D14), 4399, doi:10.1029/2002JD003186, 2003.

Dethof, A.: Monitoring of retrievals from the MIPAS and SCIAMACHY instruments on board ENVISAT, Contract Report to the European Space Agency, European Centre for Medium-Range Weather Forecasts, December 2003, available at: http://www. ecmwf.int, 2003.

Dinelli, B. M., Alpaslan, D., Carlotti, M., Magnani, L., and Ridolfi, M.: Multi-target retrieval (MTR): the simultaneous retrieval of pressure, temperature and volume mixing ratio profiles from limb-scanning atmospheric measurements, J. Quant. Spectrosc. Radiat. Transf., 84, 141-157, 2003.

Dudhia, A., Jay, V. L., and Rodgers, C. D.: Microwindow selection for high-spectral-resolution sounders, Appl. Opt., 41, 36653673, 2002a.

Dudhia, A, Morris, P. E., and Wells, R. J.: Fast monochromatic radiative transfer calculations for limb sounding, J. Quant. Spectrosc. Radiat. Transfer, 74, 745-756, 2002 b.

Dudhia, A., Jay, V.L., and Rodgers, C.D.: MIPAS Orbital Retrieval using Sequential Estimation, see websites: http://www.atm. ox.ac.uk/MORSE/ and http://www.atm.ox.ac.uk/MORSE/plan. html, 2005.

Ewen, G. B. L., Grainger, R. G., Lambert, A., and Baran, A. J.: Infrared radiative transfer modelling in a $3 \mathrm{D}$ scattering cloudy atmosphere: Application to limb sounding measurements of cirrus, J. Quant. Spectrosc. Radiat. Transf., 96, 45-74, 2005.

Farmer, C. B., Raper, O. F., and O'Callaghan, F. G.: Final Report on the First Flight of the ATMOS instrument during the Spacelab 8 mission, JPL Publication, 82-87, 1987.

Feng, W., Chipperfield, M. P., Roscoe, H. K., Remedios, J. J., Waterfall, A. M., Stiller, G. P., Glatthor, N., Höpfner, M., and Wang, D.-Y.: Three-Dimensional Model Study of the Antarctic Ozone Hole in 2002 and Comparison with 2000, J. Atmos. Sci., 62(3), 822-837, 2005.

Fischer, H. and Oelhaf, H.: Remote sensing of vertical profiles of atmospheric trace constituents with MIPAS limb-emission spectrometers, Appl. Opt., 35, 2787-2796, 1996.

Fischer, H., Blom, C., Oelhaf, H., Carli, B., Carlotti, M., Delbouille, L., Ehhalt, D., Flaud, J.-M., Isaksen, I., López-Puertas, M., McElroy, C. T., and Zander, R.: ENVISAT-MIPAS, the Michelson Interferometer for Passive Atmospheric Sounding; An instrument for atmospheric chemistry and Climate Research, ESA SP-1229, C. Readings and R. A. Harris, eds., European Space Agency, Noordwijk, The Netherlands, 2000.

Flaud, J.-M., Perrin, A., Orphal, J., Qingli K., Flaud, P.-M., Dutkiewicz, Z., and Piccolo, C.: New analysis of the $\mathrm{v}_{5}+\mathrm{v}_{9}-$ $\mathrm{v}_{9}$ hot band of $\mathrm{HNO}_{3}$, J. Quant. Spectrosc. Radiat. Transf., 77, 355-364, 2003a.

Flaud, J.-M., Wagner, G., Birk, M., Camy-Peyret, C., Claveau, C., De Backer-Barilly, M. R., Barbe, A., and Piccolo, C.: The Ozone absorption around $10 \mathrm{~mm}$, J. Geophys. Res., 108(D9), 4269, doi:10.1029/2002DJ002755, 2003b.

Flaud, J.-M., Piccolo, C., Carli, B., Perrin, A., Coudert, L. H., Teffo, J.-L., and Brown, L. R.: Molecular line parameters for the MIPAS (Michelson Interferometer for Passive Atmospheric Sounding) experiment, J. Atmos. and Ocean Opt., 16, 172-182, 2003c.
Flaud, J.-M., Brizzi, G., Carlotti, M., Perrin, A., and Ridolfi, M.: MIPAS database: Validation of $\mathrm{HNO}_{3}$ line parameters using MIPAS satellite measurements, Atmos. Chem. Phys., 6, 5037-5048, 2006a,

http://www.atmos-chem-phys.net/6/5037/2006/.

Flaud, J.-M., Brizzi, G., Carlotti, M., Perrin, A., and Ridolfi, M.: First observation of $\mathrm{H}^{15} \mathrm{NO}_{3}$ in atmospheric spectra, Geophys. Res. Lett., 34, L038025,2007.

Friedl-Vallon, F., Maucher, G., Seefeldner, M., Trieschmann, O., Kleinert, A., Lengel, A., Keim, C., Oelhaf, H., and Fischer, H.: Design and characterization of the balloon-borne Michelson Interferometer for Passive Atmospheric Sounding (MIPAS-B2), Appl. Opt., 43, 3335-3355, 2004.

Friedl-Vallon, F., Riese, M., Maucher, G., Lengel, A., Hase, F., Preusse, P., and Spang, R.: Instrument concept and preliminary performance analyses of GLORIA, Adv. Space Res., 37(12), 2287-2291, 2006.

Funke, B., López-Puertas, M., von Clarmann, T., Stiller, G. P., Fischer, H., Glatthor, N., Grabowski, U., Höpfner, M:, Kellmann, S., Kiefer, M., Linden, A., Mengistu-Tsidu, G., Milz, M., Steck, T., and Wang, D.-J.: Retrieval of stratospheric $\mathrm{NO}_{\mathrm{x}}$ from 5.3 and $6.2 \mu \mathrm{m}$ Non-LTE emissions measured by MIPAS on ENVISAT, J. Geophys. Res., 110, D09302, doi:10.1029/2004JD005225, 2005a.

Funke, B., López-Puertas, M., Gil-López, S., von Clarmann, T., Stiller, G., Kellmann, S., and Fischer, H.: Downward transport of upper atmospheric $\mathrm{NO}_{\mathrm{x}}$ into the polar stratosphere and lower mesosphere during the Antarctic 2003 and Arctic 2002/2003 winters, J. Geophys. Res., 110, D24308, doi:10.1029/2005JD006463, 2005b.

Funke, B.: for the MIPAS team: Carbon monoxide measurements from MIPAS on ENVISAT, Geophys. Res. Abstracts, 8, 10642, SRef-ID:1607-7962/gra/EGU06-A-10642, 2006.

Funke, B., López-Puertas, M., Bermejo-Pantaleón, D., von Clarmann, T., Stiller, G. P., Höpfner, M., Grabowski, U., and Kaufmann, M.: Analysis of non-local thermodynamic equilibrium CO $4.7 \mu \mathrm{m}$ fundamental, isotopic and hot band emissions measured by MIPAS on Envisat, J. Geophys. Res., 12(D11), D11305, doi:10.1029/2006JD028539, 2007.

Gardner, J. L., López-Puertas, M., Funke, B., Miller, S. M., Lipson, S. J., and Sharma, R. D.: Rotational and spin-orbit distributions of NO observed by MIPAS/ENVISAT during the solar storm of October/November 2003, J. Geophys. Res., 110, A09S34, doi:10.1029/2004JA010937, 2005.

Glatthor, N., von Clarmann, T., Fischer, H., Grabowski, U., Höpfner, M., Kellmann, S., Kiefer, M., Linden, A., Milz, M., Steck, T., Stiller, G. P., Mengistu Tsidu, G., Wang, D.-Y., and Funke, B.: Spaceborne $\mathrm{ClO}$ observations by the Michelson Interferometer for Passive Atmospheric Sounding (MIPAS) before and during the Antarctic major warming in September/October 2002, J. Geophys. Res., 109(D11), D11307, doi:10.1029/2003JD004440, 2004.

Glatthor, N., von Clarmann, T., Fischer, H., Funke, B., Grabowski, U., Höpfner, M., Kellmann, S., Kiefer, M., Linden, A., Milz, M., Steck, T., Stiller, G. P., Mengistu Tsidu, G., and Wang, D.-Y.: Mixing processes during the Antarctic vortex split in September/October 2002 as inferred from source gas and ozone distributions from MIPAS/ENVISAT, J. Atm. Sci., Special issue on Antarctic Vortex, 62(3), 787-800, 2005. 
Glatthor, N., von Clarmann, T., Fischer, H., Funke, B., Grabowski, U., Höpfner, M., Kellmann, S., Kiefer, M., Linden, A., Milz, M., Steck, T., and Stiller, G.: Retrieval of peroxyacetylnitrate (PAN) in the upper troposphere from MIPAS level-1B spectra, Poster presentation, Atmospheric Science Conference, ESRIN, Frascati, 8-12 May 2006, available online http://earth.esa.int/cgi-bin/ confatmos06.pl?abstract=865, 2006.

Greenhough, J., Remedios, J. J., Sembhi, H., and Kramer, L. J.: Towards cloud detection and cloud frequency distributions from MIPAS infra-red observations, Adv. Space Res., 36(5), 800-806, doi:10.1016/j.asr.2005.04.096, 2005.

Gunson, M. R., Abbas, M. M., Abrams, M. C., Allen, M., Brown, L. R., Brown, T. L., Chang, A. Y., Goldman, A., Irion, F. W., Lowes, L. L., Mahieu, E., Manney, G. L., Michelsen, H. A., Newchurch, M. J., Rinsland, C. P., Salawitch, R. J., Stiller, G. P., Toon, G. C., Yung, Y. L., and Zander, R.: The Atmospheric Trace Molecule Spectroscopy (ATMOS) experiment: Deployment on the ATLAS Space Shuttle missions, Geophys. Res. Lett., 23(17), 2333-2336, 1996.

Hoffmann, L., Spang, R., Kaufmann, M., and Riese, M.: Retrieval of CFC-11 and CFC-12 from ENVISAT MIPAS observations by means of rapid radiative transfer calculations, Adv. Space Res., 36(5), 915-921, 2005.

Höpfner, M., Oelhaf, H., Wetzel, G., Friedl-Vallon, F., Kleinert, A., Lengel, A., Maucher, G., Nordmeyer, H., Glatthor, N., Stiller, G., von Clarmann, T., and Fischer, H.: Evidence of scattering of tropospheric radiation by PSCs in mid-IR limb emission spectra: MIPAS-B observations and KOPRA simulations, Geophys. Res. Lett., 29(8), 1278, doi:10.1029/2001GL014443, 2002.

Höpfner, M., von Clarmann, T., Fischer, H., Glatthor, N., Grabowski, U., Kellmann, S., Kiefer, M., Linden, A., Mengistu Tsidu, G., Milz. M., Steck, T., Stiller, G. P., Wang, D.-Y., and Funke, B.: First spaceborne observations of Antarctic stratospheric $\mathrm{ClONO}_{2}$ recovery: Austral spring 2002, J. Geophys. Res., 109, D11308, doi:10.1029/2004JD004609, 2004a.

Höpfner, M.: Study on the impact of polar stratospheric clouds on high resolution mid-IR limb emission spectra, J. Quant. Spectrosc. Radiat. Transfer, 83(1), 93-107, doi:10.1016/S00224073(02)00299-6, 2004b.

Höpfner, M. and Emde, C.: Comparison of single and multiple scattering approaches for the simulation of limb-emission observations in the mid-IR, J. Quant. Spectrosc. Radiat. Transfer, 91(3), 275-285, doi:10.1016/j.jqsrt.2004.05.066, 2005.

Höpfner, M., Luo, B. P., Massoli, P., Cairo, F., Spang, R., Snels, M., Di Donfrancesco, G., Stiller, G., von Clarmann, T., Fischer, H., and Biermann, U.: Spectroscopic evidence for NAT, STS, and ice in MIPAS infrared limb emission measurements of polar stratospheric clouds, Atmos. Chem. Phys., 6, 1201-1219, 2006a, http://www.atmos-chem-phys.net/6/1201/2006/.

Höpfner, M., Larsen, N., Spang, R., Luo, B. P., Ma, J., Svendsen, S. H., Eckermann, S. D., Knudsen, B., Massoli, P., Cairo, F., Stiller, G., von Clarmann, T., and Fischer, H.: MIPAS detects Antarctic stratospheric belt of NAT PSCs caused by mountain waves, Atmos. Chem. Phys., 6, 1221-1230, availobe at: http: //direct.sref.org/1680-7324/acp/2006-6-1221, 2006b.

Jacquinet-Husson, N., Scott, N. A., Chédin, A., Garceran, K., Armante, R., Chursin, A .A., Barbe, A., Birk, M., Brown, L. R., Camy-Peyret, C., Claveau, C., Clerbaux, C., Coheur, P. F., Dana, V., Daumont, L., Debacker-Barilly, M. R., Flaud, J.-M., Gold- man, A., Hamdouni, A., Hess, M., Jacquemart, D., Köpke, P., Mandin, J. Y., Massie, S., Mikhailenko, S., Nemtchinov, V., Nikitin, A., Newnham, D., Perrin, A., Perevalov, V. I., RegaliaJarlot, L., Rublev, A., Schreier, F., Schult, L., Smith, K. M., Tashkun, S. A., Teffo, J. L., Toth, R. A.,. Tyuterev, VL. G, Vander Auwera, J., Varanasi, P., and Wagner, G.: The 2003 edition of the GEISA/IASI spectroscopic database, J. Quant. Spectrosc. Radiat. Transfer, 95, 429-467, doi:10.1016/j.jqsrt.2004.12.004, 2005.

Juckes, M. N.: Evaluation of MIPAS ozone fields assimilated using a new algorithm constrained by isentropic tracer advection, Atmos. Chem. Phys., 6, 1549-1565, availibe at: http://direct.sref. org/1680-7324/acp/2006-6-1549, 2006.

Kalogerakis, K. S., Copeland, R. A., and Slanger, T. G.: Measurement of the rate coefficient for collisional removal of $\mathrm{O}_{2}\left(\mathrm{X}^{3} \Sigma_{g}^{-}\right.$, $v=1)+\mathrm{O}\left({ }^{3} \mathrm{P}\right)$, J. Chem. Phys., 123, 194-303, 2005.

Kaye, J. A. and Kumer, J. B.: Nonlocal thermodynamic equilibrium effects in stratospheric NO and implications for infrared remote sensing, Appl. Opt., 26(22), 4747-4754, 1987.

Kaufmann, M., Gil-López, S., Funke, B., García-Comas, M., Koukouli, M. E., López-Puertas, M., Glatthor, N., Grabowski, U., Höpfner, M., Stiller, G. P., von Clarmann, T., and Riese, M.: Vibrationally excited ozone in the middle atmosphere, J. Atmos. Sol. Terr. Phys., doi:10.1016/j.jastp.2005.10.006, 68, 202-212, 2006.

Keim, C., Blom, C. E.; von der Gathen, P., Gulde, T., Höpfner, M., Liu, G. Y., Oulanovski, A., Piesch, C., Ravegnani, F., Sartorius, C., Schlager, H., and Volk, C. M.: Validation of MIPASENVISAT by correlative measurements of MIPAS-STR. Proc. ACVE-2 meeting, 3-7 May 2004, Frascati, Italy, ESA SP-562, 2004.

Kiefer, M., von Clarmann, T., Grabowski, U., De Laurentis, M., Mantovani, R., Milz, M., and Ridolfi, M.: Characterization of MIPAS elevation pointing, Atmos. Chem. Phys., 7, 1615-1628, availible at: http://direct.sref.org/1680-7324/acp/2007-7-1615, 2007.

Kleinert, A., Aubertin, G., Perron, G., Birk, M., Wagner, G., Hase, F., Nett, H., and Poulin, R.: MIPAS Level 1B Algorithms Overview: Operational Processing And Characterization, Atmos. Chem. Phys., 7, 1395-1406, availible at: http://direct. sref.org/1680-7324/acp/2007-7-1395, 2007.

Koukouli, M. E., López-Puertas, M., Gil-López, S., Funke, B., and Milz, M.: for the IAA/IMK MIPAS/ENVISAT team: Water vapour profiles and Non-LTE parameters from its mesospheric emissions derived from MIPAS/ENVISAT, Geophys. Res. Abstracts, 8, 03303, SRef-ID: 1607-7962/gra/EGU06-A03303, 2006.

Konopka, P., Engel, A., Funke, B., Mueller, R., Grooss, J.U. ,Guenther, G., Wetter, T., Stiller, G., von Clarmann, T., Glatthor, N., Oelhaf, H., Wetzel, G., López-Puertas, M., Pirre, M., Huret, N., and Riese, M.: Ozone loss driven by nitrogen oxides and triggered by stratospheric warmings can outweigh the effect of halogens, J. Geophys. Res., 112, D05105, doi:10.1029/2006JD007064, 2007.

Kouker, W.: Towards the Prediction of Stratospheric Ozone (TOPOZ III), EC Contract No EVK2-CT-2001-00102, Scientific Report, 2005.

Kramer, L. J.: Sensitivity of the MIPAS instrument on ENVISAT to the detection of Polar Stratospheric Clouds, M. Phil. thesis, 
University of Leicester, 2004.

Levenberg, K.: A method for the solution of certain problems in least squares, Quart. Appl. Math. 2, 164-168, 1944.

Lahoz, W. A., Geer, A. J., Bekki, S., Bormann, N., Ceccherini, S., Elbern, H., Errera, Q., Eskes, H. J., Fonteyn, D., Jackson, D. R., Khattatov, B., Massart, S., Peuch, V.-H., Rharmili, S., Ridolfi, M., Segers, A., Talagrand, O., Thornton, H. E., Vik, A. F., and von Clarmann, T.: The Assimilation of ENVISAT data (ASSET) project, Atmos. Chem. Phys., 7, 1773-1796, 2007,

http://www.atmos-chem-phys.net/7/1773/2007/.

López-Puertas, M. and Taylor, F. W.: Non-LTE Radiative Transfer in the Atmosphere, Series on Atmospheric, Oceanic and Planetary Physics, Vol. 3, World Scientific Publishing Co. Pte. Ltd., ISBN 981-02-4566-1, 2001.

López-Puertas, M., Funke, B., Gil-López, S., von Clarmann, T., Stiller, G. P., Kellmann, S., Fischer, H., and Jackman, C. H.: Observation of $\mathrm{NO}_{\mathrm{x}}$ Enhancement and Ozone Depletion in the Northern and Southern hemispheres after the October-November 2003 Solar Proton Events, J. Geophys. Res., 110, A09S43, doi:10.1029/2005JA011050, 2005a.

López-Puertas, M., Funke, B., Gil-López, S., von Clarmann, T., Stiller, G. P., Höpfner, M., Kellmann, S., Mengistu Tsidu, G., Fischer, H., and Jackman, C. H.: $\mathrm{HNO}_{3}$, $\mathrm{N}_{2} \mathrm{O}_{5}$ and $\mathrm{ClONO}_{2}$ Enhancements after the October-November 2003 Solar Proton Events, J. Geophys. Res., 110, A09S44, doi:10.1029/2005JA011051, 2005b.

López-Puertas, M., Funke, B., Gil-López, S., López-Valverde, M. A., von Clarmann, T., Fischer, H., Oelhaf, H., Stiller, G., Kaufmann, M., Koukouli, M. E., and Flaud, J.-M.: Atmospheric non-Local Thermodynamic Equilibrium Emissions as Observed by the Michelson Interferometer for Passive Atmospheric Sounding (MIPAS), C. R. Physique, 8, 848-863, doi:10.1016/j.crhy.2005.07.012, 2005c.

López-Puertas, M., Koukouli, M. E., Funke, B., Gil-López, S., Glatthor, N., Grabowski, U., von Clarmann, T., and Stiller, G. P.: Evidence for $\mathrm{CH}_{4} 7.6 \mu \mathrm{m}$ non-local thermodynamic equilibrium emission in the mesosphere, Geophys. Res. Lett., 32, L04805, doi:10.1029/2004GL021641, 2005d.

López-Puertas, M., Funke, B., Gil-López, S., von Clarmann, T., Fischer, H., Glatthor, N., Grabowski, U., Höpfner, M., Kellmann, S., Kiefer, M., Linden, A., Milz, M., Reddmann, T., Steck, T., Stiller, G. P., and Sinnhuber, M.: Composition Changes Caused by the 2003 Solar Storm: MIPAS Measurements and Model Simulations, ENVISAT Atmosp. Sci. Conf., ESRIN, Frascati, 2006.

López-Puertas, M., Funke, B., von Clarmann, T., Fischer, H., and Stiller, G.: The stratospheric and mesospheric $\mathrm{NO}_{\mathrm{y}}$ in the 20022004 polar winters as measured by MIPAS/ENVISAT, Space Sci. Rev., 125, 403-416, doi:10.1007/s11214-006-9073-2, 2007a.

López-Puertas, M., Funke, B., Bermejo-Pantaleón, D., von Clarmann, T. Stiller, G. P., Grabowski, U., and Höpfner, M.: Evidence for $\mathrm{N}_{2} \mathrm{O} v_{3} 4.5 \mu \mathrm{m}$ non-local thermodynamic equilibrium emission in the atmosphere, Geophys. Res. Lett., 34(2), L02825, doi:10.1029/2006GL028539, 2007b.

López-Valverde, M. A., López-Puertas, M., Remedios, J. J., Rodgers, C. D., Taylor, F. W., Zipf, E. C., and Erdman, P. W.: Validation of Measurements of Carbon Monoxide from the Improved Stratospheric and Mesospheric Sounder, J. Geophys. Res., 101, 9929-9955, 1996.

Manney, G. L., Sabutis, J. L., Allen, D. R., Lahoz, W. A., Scaife, A.
A., Randall, C. E., Pawson, S., Naujokat, B., and Swinbank, R.: Simulations of Dynamics and Transport During the September 2002 Antarctic Major Warming, J. Atmos. Sci., 62(3), 690-707, 2005.

McKee, T. B., Whitman, R. I., and Lambiotte Jr., J. J.: A technique to infer atmospheric water-vapor mixing ratio from measured horizon radiance profiles, NASA TN D-5252, 1969.

Mencaraglia, F., Bianchini, G., Boscaleri, A., Carli, B., Ceccherini, S., Raspollini, P., Perrin, A., and Flaud, J.-M.: Validation of MIPAS satellite measurements of $\mathrm{HNO}_{3}$ using comparison of rotational and vibrational spectroscopy, J. Geophys. Res., 110, D19305, doi:1029/2005JD006099, 2006.

Mengistu Tsidu, G., von Clarmann, T., Stiller, G., Höpfner, M., Fischer, H., Funke, B., Glatthor, N., Grabowski, U., Höpfner, M., Kellmann, S., Kiefer, M., Linden, A., Milz, M., Steck, T., and Wang, D.-Y.: Stratospheric $\mathrm{N}_{2} \mathrm{O}_{5}$ in the Austral Spring 2002 as retrieved from limb emission spectra recorded by the Michelson Interferometer for Passive Atmospheric Sounding (MIPAS), J. Geophys. Res., 109, D18301, doi:10.1029/2004JD004856, 2004.

Mengistu Tsidu, G., Stiller, G. P., von Clarmann, T., Funk,e, B., Fischer, H., Glatthor, N., Grabowski, U., Höpfner, M., Kellmann, S., Kiefer, M., Linden, A., Milz, M., Steck, T., and Wang, D.-Y.: $\mathrm{NO}_{\mathrm{y}}$ from Michelson Interferometer for Passive Atmospheric Sounding on Environmental Satellite during the Southern Hemisphere Polar Vortex Split in September/October 2002, J. Geophys. Res., 110(D11), D11301, doi:10.1029/2004JD005322, 2005.

Menke, W.: Geophysical Data Analysis: Discrete Inverse Theory, Academic, San Diego, California, 1984.

Milz, M., von Clarmann, T., Fischer, H., Glatthor, N., Grabowski, U., Höpfner, M., Kellmann, S., Kiefer, M., Linden, A., Mengistu Tsidu, G., Steck, T., Stiller, G. P., Funke, B., and López-Puertas, M.: Water vapour distributions Measured with the Michelson Interferometer for Passive Atmospheric Sounding on board ENVISAT (MIPAS/ENVISAT), J. Geophys. Res., 110, D24307, doi:10.1029/2005JD005973, 2005.

Moore, D. P.: Measurements of HCFC-22 in the upper troposphere and lower stratosphere from the MIPAS-E instrument, Ph.D. thesis, University of Leicester, 2005.

Moore, D. P., Waterfall, A. M., and Remedios, J. J.: The potential for radiometric retrievals of halocarbon concentrations from the MIPAS-E instrument, Adv. Space Res., 37(12), 2238-2246, 2006.

Nedoluha, G. E., Bevilacqua, R. M., Gomez, R. M., Hicks, B. C., Russell III, J. M., and Connor, B.: Ground-based microwave observations of middle atmospheric water vapour in the 1990s, in Atmospheric Science Across the Stratopause, edited by: Siskind, D.E., Eckermann, S.D., Summers, M.E., et al., Am. Geophys. Union, Geophys. Monograph 123, 257-270, 2000.

Papandrea, E., Dudhia, A., Grainger, R. G., Vancassel, X., and Chipperfield, M. P.: Retrieval of Hydrogen Peroxide $\left(\mathrm{H}_{2} \mathrm{O}_{2}\right)$ Profiles using ENVISAT-MIPAS, Geophys. Res. Lett., 32, L14809, doi:10.1029/2005GL022870, 2005.

Payan, S., Camy-Peyret, C., Oelhaf, H., Wetzel, G., Maucher, G., Keim, C., Pirre, M., Engel, A., Volk, M. C., Kuttippurath, J., Cortesi, U., Raspollini, P., Vigouroux, C., De Maziere, M., Piccolo, C., Payne, V., Bracher, A., Glatthor, N., Stiller, G., Grunow, K., Butz, A.: Validation and data characteristics of methane and nitrous oxide profiles observed by MIPAS and processed with 
Version 4.61 algorithm, Atmos. Chem. Phys. Discuss., 7, 18043$18111,2007$.

Randall, C. E., Harvey, V.L., Manney, G.L., et al.: Stratospheric effects of energetic particle precipitation in 2003-2004, Geophys. Res. Lett., 32, L05802, doi:10.1029/2004GL022003, 2005.

Raspollini, P., Belotti, C., Burgess, A., Carli, B., Carlotti, M., Ceccherini, S., Dinelli, B. M., Dudhia, A., Flaud, J.-M., Funke, B., Höpfner, M., López-Puertas, M., Payne, V., Piccolo, C., Remedios, J. J., Ridolfi, M., and Spang, R.: MIPAS level 2 operational analysis, Atmos. Chem. Phys., 6, 5605-5630, 2006,

http://www.atmos-chem-phys.net/6/5605/2006/.

Remedios, J. J., Allen, G., and Waterfall, A. M.: Infra-red remote sensing of organic compounds in the upper troposphere, Proceedings of the ESA Atmospheric Science Conference, ESRIN, Rome, May 2006.

Ridolfi, M., Carli, B., Carlotti, M., von Clarmann, T., Dinelli, B. M., Dudhia, A., Flaud, J.-M., Höpfner, M., Morris, P. E., Raspollini, P., Stiller, G., and Wells, R. J.: Optimized forward model and retrieval scheme for MIPAS near-real-time data processing, Appl. Opt., 39, 1323-1340, 2000.

Ridolfi, M., Alpaslan. D., Carli, B., Carlotti, M., Castelli, E., Ceccherini, S., Dinelli, B. M., Dudhia. A., Flaud, J.-M., Höpfner, M., Jay, V., Magnani, L., Oelhaf, H., Payne, V., Piccolo, C., Prosperi, M., Raspollini, P., Remedios, J. J., and Spang, R.: MIPAS level 2 processor performance and verification, in Proceedings of the ENVISAT Validation Workshop of European Space Research Institute, 9-13 November 2002, ESRIN, Frascati, Italy, ESA SP-531 (http://envisat.esa.int/pub/ESA_DOC/ envisat_val_1202/proceedings/), 2002.

Ridolfi, M., Magnani, L., Carlotti, M., and Dinelli, B. M.: MIPASENVISAT limb-sounding measurements: Trade-of study for improvement of horizontal resolution, Appl. Opt., 43(31), 58145824, 2004.

Ridolfi, M., Blum, U., Carli, B., Catoire, V., Ceccherini, S., Claude, H., De Clercq, C., Fricke, K. H., Friedl-Vallon, F., Iarlori, M., Keckhut, P., Kerridge, B., Lambert, J.-C., Meijer, Y. J., Mona, L, Oelhaf, H., Pappalardo, G., Pirre, M., Rizi, V., Robert, C., Swart, D., von Clarmann, T., Waterfall, A., and Wetzel, G.: Geophysical validation of temperature retrieved by the ESA processor from MIPAS/ENVISAT atmospheric limb-emission measurements, Atmos. Chem. Phys., 7, 4459-4487, availible at: http://direct.sref.org/1680-7375/acpd/2007-7-5439, 2007.

Riese, M., Friedl-Vallon, F., Spang, R., Preusse. P., Schiller, C., Hoffmann, L., Konopka, P., Oelhaf, H., von Clarmann, T, and Höpfner, M.: GLObal limb Radiance Imager for the Atmosphere (GLORIA): Scientific objectives, Adv. Space Res., 36(5), 989995, 2005.

Rinsland, C. P., Gunson, M.R., Wang, P.H., et al.: ATMOS/ATLAS3 infrared profile measurements of tracegases in the November 1994 tropical and subtropical upper troposphere, J. Quant. Spectrosc. Radiat. Transf., 60, 891-901, 1998.

Rinsland C. P., Boone, C., Nassar, R., Walker, K., Bernath, P., McConnell, J. C., and Chiou, L.: Atmospheric Chemistry Experiment (ACE) Arctic stratospheric measurements of $\mathrm{NO}_{\mathrm{x}}$ during February and March 2004: Impact of intense solar flares, Geophys. Res. Lett., 32, L16S05, doi:10.1029/2005GL022425, 2005a.

Rinsland C. P., Dufour, G., Boone, C. D., Bernath, P. F., and Chiou, L.: Atmospheric Chemistry Experiment (ACE) measurements of elevated Southern Hemisphere upper tropospheric $\mathrm{CO}, \mathrm{C}_{2} \mathrm{H}_{6}$, $\mathrm{HCN}$, and $\mathrm{C}_{2} \mathrm{H}_{2}$ mixing ratios from biomass burning emissions and long-range transport, Geophys. Res. Lett., 32, L20803, doi:10.1029/2005GL024214, 2005b.

Rodgers C. D.: Retrieval of atmospheric temperature and composition from remote measurements of thermal radiation. Rev. Geophys. Space Phys., 14(4), 609-624, 1976.

Rodgers, C. D.: Inverse methods for atmospheric sounding - Theory and practise, edited by: Taylor, F. W., World Scientific, Singapore, 2000.

Rothman, L. S., Jacquemart, D., Barbe, A., Benner, D. C., Birk, M., Brown, L. R., Carleer, M. R., Chackerian, C., Chance, K., Coudert, L. H., Dana, V., Devi, V. M., Flaud, J. M., Gamache, R. R., Goldman, A., Hartmann, J. M., Jucks, K. W., Maki, A. G., Mandin, J. Y., Massie, S. T., Orphal, J., Perrin, A., Rinsland, C. P., Smith, M. A. H., Tennyson, J., Tolchenov, R. N., Toth, R. A., Vander Auwera, J., Varanasi, P., and Wagner, G.: The HITRAN 2004 molecular spectroscopic database, J. Quantitative Spectroscopy Radiative Transfer, 96(2), 139-204, doi:10.1016/j.jqsrt.2004.10.008, 2005.

Schwinger, J., Elbern, H., and Botchorishvili, R.: A new 4dimensional variational assimilation system applied to ENVISAT MIPAS observation, Proc. of the 2004 ENVISAT and ERS Symposium, 6-10 September 2004, Salzburg, Austria, (ESA SP-572, April 2005) CD-ROM, ISBN 92-9092-883-2, ISSN 1609-042X, 2005.

Sinnhuber, B.-M., Rozanov, A., Sheode, N., Afe, O. T., Richter, A., Sinnhuber, M., Wittrock, F., Burrows, J. P., Stiller, G. P., von Clarmann, T., and Linden, A.: Global observations of stratospheric bromine monoxide from SCIAMACHY, Geophys. Res. Lett., 32(20), L20810, doi:10.1029/2005GL023839, 2005.

Siskind, D. E., Eckerman, S. D., and Summers, M. E. (Eds.): Atmospheric Science Across the Stratopause, Am. Geophys. Union, Geophys. Monograph 123, Washington, 2000.

Sivia, D. S.: Data analysis, A Bayesian tutorial, Clarendon Press, Oxford, 1998.

Smith, A. K. and Marsh, D. R.: Processes that account for the ozone maximum at the mesopause, J. Geophys. Res., 110, D23305, doi:10.1029/2005JD006298, 2005.

Spang, R., and Remedios, J. J.: Observations of a distinctive infrared spectral feature in the atmospheric spectra of polar stratospheric clouds measured by the CRISTA instrument, Geophys. Res. Lett., 30(16), 1875, doi:10.1029/2003GL017231, 2003.

Spang, R., Remedios, J. J., and Barkley, M. P.: Colour indices for the detection and differentiation of cloud types in infra-red limb emission spectra, Adv. Space Res., 33(7), 1041-1047, 2004.

Spang, R., Remedios, J. J., Kramer, L. J., Poole, L. R., Fromm, M. D., Müller, M., Baumgarten, G., and Konopka, P.: Polar stratospheric cloud observations by MIPAS on ENVISAT: detection method, validation and analysis of the northern hemisphere winter 2002/2003, Atmos. Chem. Phys., 5, 679-692, availible at: http://direct.sref.org/1680-7324/acp/2005-5-679, 2005a.

Spang, R., Remedios, J. J., Tilmes, S., and Riese, M.: MIPAS observations of Polar Stratospheric Clouds in the Arctic 2002/3 and Antarctic 2003 winters, Adv. Space Res., 36, 868-878, 2005 b.

Steck, T. and von Clarmann, T.: Constrained Profile Retrieval Applied to the Observation Mode of the Michelson Interferometer for Passive Atmospheric Sounding, Appl. Opt., 40(21), 35593571, 2001. 
Steck, T., Höpfner, M., von Clarmann, T., and Grabowski, U.: Tomographic retrieval of atmospheric parameter from infrared limb emission observations, Appl. Opt., 44(16), 3291-3301, 2005.

Steinwagner, J., Milz, M., Clarmann, T. von, Glatthor, N., Grabowski, U., Höpfner, M., Stiller, G.P., Röckmann, T.: HDO measurements with MIPAS, Atmospheric Chemistry and Physics, 7, 2601-2615, 2007.

Stiller, G. P. (Editor) with contributions from von Clarmann, T., Dudhia, A., Echle, G., Funke, B, Glatthor, N.,. Hase, F, Höpfner, M, Kellmann, S, Kemnitzer, H., Kuntz, M., Linden, A., Linder, M., Stiller, G. P., and Zorn, S.: The Karlsruhe Optimized and Precise Radiative transfer Algorithm (KOPRA), Forschungszentrum Karlsruhe, Wissenschaftliche Berichte, Bericht Nr. 6487, 2000.

Stiller, G. P., Mengistu Tsidu, G., von Clarmann, T., Glatthor, N., Höpfner, M., Kellmann, S., Linden, A., Ruhnke, R., Fischer, H., López-Puertas, M., Funke, B., and Gil-López, S.: An enhanced $\mathrm{HNO}_{3}$ second maximum in the Antarctic midwinter upper stratosphere 2003, J. Geophys. Res., 110, D20303, doi:10.1029/2005JD006011, 2005.

Stiller, G. P., von Clarmann, T., Höpfner, M., Glatthor, N., Grabowski, U., Kellmann, S, Kleinert, A., Linden, A., Milz, M., Reddmann, T., Steck, T., Fischer, H., Funke, B., López-Puertas, M., and Engel, A.: Global distribution of mean age of stratospheric air from MIPAS SF 6 measurements, Atmos. Chem. Phys. Discuss. 7, 13653-13679, 2007a.

Stiller, G. P., von Clarmann, T., Brühl, Ch., Fischer, H., Funke, B., Glatthor, N., Grabowski, U., Höpfner, M., Jöckel, P., Kellmann, S., Kiefer, M., Linden, A., López-Puertas, M., Mengistu Tsidu, G., Milz, M., Steck, T., and Steil, B.: Global distributions of $\mathrm{HO}_{2} \mathrm{NO}_{2}$ as observed by the Michelson Interferometer for Passive Atmospheric Sounding (MIPAS), J. Geophys. Res., 112, D09314, doi:10.1029/2006JD007212, 2007b.

Tikhonov, A.: On the solution of incorrectly stated problems and method of regularization, Dokl. Akad. Nauk. SSSR, 153(3), 501504, 1963a.

Tikhonov, A.: On the regularization of incorrectly stated problems, Dokl. Akad. Nauk. SSSR, 153(1), 49-52, 1963 b.

von Clarmann, T. and Echle, G.: Selection of Optimized Microwindows for Atmospheric Spectroscopy, Appl. Opt., 37 (33), 7661 7669, 1998.

von Clarmann, T., Glatthor, N., Grabowski, U., Höpfner, M., Kellmann, S., Kiefer, M., Linden, A., Mengistu Tsidu, G., Milz, M., Steck, T., Stiller, G. P., Wang, D.-Y., Fischer, H., Funke, B., Gil-López, S., and López-Puertas, M.: Retrieval of temperature and tangent altitude pointing from limb emission spectra recorded from space by the Michelson Interferometer for Passive Atmospheric Sounding (MIPAS), J. Geophys. Res., 108, doi:10.1029/2003JD003602, 2003a.

von Clarmann, T., Chidiezie Chineke, T., Fischer, H., Funke, B., Garcia-Comas, M., Gil-López, S., Glatthor, N., Grabowski, U., Höpfner, M., Kellmann, S., Kiefer, M., Linden, A., LópezPuertas, M., López-Valverde, M. A., Mengistu Tsidu, G., Milz, M., Steck, T., and Stiller, G. P.: Remote Sensing of the Middle Atmosphere with MIPAS, in: Remote Sensing of Clouds and the Atmosphere VII, K. Schäfer, O. Lado-Bordowsky, edited by: Comerón, A. and Picard, R. H., Proceedings of SPIE Vol. 4882, SPIE, Bellingham, WA, USA, 172-183, 2003 b.

von Clarmann, T., Höpfner, M., Funke, B., López-Puertas, M., Dud- hia, A., Jay, V., Schreier, F., Ridolfi, M., Ceccherini, S., Kerridge, B., Reburn, J., Siddans, R., and Flaud, J.-M.: Modelling of atmospheric mid-infrared radiative transfer: the AMIL2DA algorithm intercomparison experiment, J. Quant. Spectrosc. Radiat. Transf., 78(3-4), 381-407, 2003c

von Clarmann, T., Glatthor, N., Höpfner, M., Kellmann, S., Ruhnke, R., Stiller, G. P., Fischer, H., Funke, B., Gil-López, S., and López-Puertas, M.: Experimental evidence of perturbed odd hydrogen and chlorine chemistry after the October 2003 solar proton events, J. Geophys. Res., 110(A9), A09S45, doi:10.1029/2005JA011053, 2005.

von Clarmann, T., Glatthor, N., Grabowski, U., Höpfner, M., Kellmann, S., Linden, A., Mengistu Tsidu, G., Milz, M., Steck, T., Stiller, G. P., Fischer, H., and Funke, B.: Global stratospheric $\mathrm{HOCl}$ distributions retrieved from infrared limb emission spectra recorded by the Michelson Interferometer for Passive Atmospheric Sounding (MIPAS), J. Geophys. Res., 111, D05311, doi:10.1029/2005JD005939, 2006a.

von Clarmann, T.: Validation of remotely sensed profiles of atmospheric state variables: strategies and terminology, Atmos. Chem. Phys., 6, 4311-4320, availible at: http://direct.sref.org/ 1680-7324/acp/2006-6-4311, 2006b.

Wamsley, P. R., Elkins, J.W., Fahey, D.W., et al.: Distribution of halon-1211 in the upper troposphere and lower stratosphere and the 1994 total bromine budget, J. Geophys. Res., 103, 15131526, 1998.

Wang, D.-Y., von Clarmann, T., Fischer, H., Funke, B., Gil-López, S., Glatthor, N., Grabowski, U., Höpfner, M., Kaufmann, M., Kellmann, S., Kiefer, M., Koukouli, M. E., Linden, A., LópezPuertas, M., Mengistu Tsidu, G., Milz, M., Steck, T., Stiller, G. P. , Simmons, A. J., Dethof, A., Swinbank, R., Marquardt, C., Jiang, J. H., Romans, L. J., Wickert, J., Schmidt, T., Russell III, J., and Remsberg, E.: Validation of stratospheric temperatures measured by MIPAS on ENVISAT, J. Geophys. Res., 110(D8), D08301, 10.1029/2004JD005342, 2005a.

Wang, D.-Y., von Clarmann, T., Fischer, H., Funke, B., GarcíaComas, M., Gil-López, S., Glatthor, N., Grabowski, U., Höpfner, M., Kellmann, S., Kiefer, M., Linden, A., López-Puertas, M., López-Valverde, M. A., Mengistu Tsidu, G., Milz, M., Steck, T., and Stiller, G. P.: Longitudinal variations of temperature and ozone profiles observed by MIPAS during the Antarctic Stratosphere Sudden Warming of 2002, J. Geophys. Res., 110(D20), D20101, doi:10.1029/2004JD005749, 2005b.

Wang, D. Y., Höpfner, M., Blom, C. E., Ward, W. E., Fischer, H., Blumenstock, T., Hase, F., Keim, C., Liu, G. Y., Mikuteit, S., Oelhaf, H., Wetzel, G., Cortesi, U., Mencaraglia, F., Bianchini, G., Redaelli, G., Pirre, M., Catoire, V., Huret, N., Vigouroux, C., De Mazière, M., Mahieu, E., Demoulin, P., Wood, S., Smale, D., Jones, N., Nakajima, H., Sugita, T., Urban, J., Murtagh, D., Boone, C. D., Bernath, P. F., Walker, K. A., Kuttippurath, J., Kleinböhl, A., Toon, G., and Piccolo, C.: Validation of MIPAS $\mathrm{HNO}_{3}$ operational data, Atmos. Chem. Phys., 7, 4905-4934, availible at: http://direct.sref.org/1680-7375/acpd/2007-7-5173, 2007.

Waterfall, A. M., Remedios, J. J., Allen, G., and Sembhi, H.: Infrared remote sensing of organic compounds in the upper troposphere, Proceedings of the ENVISAT Symposium, Salzburg, September, 2004. 
Weatherhead, E. C. and Andersen, S. B.: The search for signs of recovery of the ozone layer, Nature, 441, 39-45, doi:10.1038/nature04746, 2006.

Wetzel, G., Bracher, A., Funke, B., Goutail, F., Hendrick, F., Lambert J.-C., Mikuteit, S., Piccolo, C., Pirre, M., Bazureau, A., Belotti, C., Blumenstock, T., De Mazière, M., Fischer, H., Huret,
N., Ionov, D., López-Puertas, M., Maucher, G., Oelhaf, H., Pommereau, J.-P., Ruhnke, R., Sinnhuber, M., Stiller, G., Van Roozendael, M., and Zhang, G.: Validation of MIPAS-ENVISAT $\mathrm{NO}_{2}$ operational data, Atmos. Chem. Phys., 7, 3261-3284, 2007, http://www.atmos-chem-phys.net/7/3261/2007/. 